ESAIM: COCV 20 (2014) 269-314

DOI: $10.1051 / \mathrm{cocv} / 2013064$
ESAIM: Control, Optimisation and Calculus of Variations

www.esaim-cocv.org

\title{
PERIODIC STABILIZATION FOR LINEAR TIME-PERIODIC ORDINARY DIFFERENTIAL EQUATIONS *,**
}

\author{
Gengsheng WAnG ${ }^{1}$ And Yashan XU ${ }^{2}$
}

\begin{abstract}
This paper studies the periodic feedback stabilization of the controlled linear time-periodic ordinary differential equation: $\dot{y}(t)=A(t) y(t)+B(t) u(t), t \geq 0$, where $[A(\cdot), B(\cdot)]$ is a $T$-periodic pair, i.e., $A(\cdot) \in L^{\infty}\left(\mathbb{R}^{+} ; \mathbb{R}^{n \times n}\right)$ and $B(\cdot) \in L^{\infty}\left(\mathbb{R}^{+} ; \mathbb{R}^{n \times m}\right)$ satisfy respectively $A(t+T)=A(t)$ for a.e. $t \geq 0$ and $B(t+T)=B(t)$ for a.e. $t \geq 0$. Two periodic stablization criteria for a $T$-period pair $[A(\cdot), B(\cdot)]$ are established. One is an analytic criterion which is related to the transformation over time $T$ associated with $A(\cdot)$; while another is a geometric criterion which is connected with the null-controllable subspace of $[A(\cdot), B(\cdot)]$. Two kinds of periodic feedback laws for a $T$-periodically stabilizable pair $[A(\cdot), B(\cdot)]$ are constructed. They are accordingly connected with two Cauchy problems of linear ordinary differential equations. Besides, with the aid of the geometric criterion, we find a way to determine, for a given $T$-periodic $A(\cdot)$, the minimal column number $m$, as well as a time-invariant $n \times m$ matrix $B$, such that the pair $[A(\cdot), B]$ is $T$-periodically stabilizable.
\end{abstract}

Mathematics Subject Classification. 34H15, 49N20.

Received October 23, 2012.

Published online January 27, 2014.

\section{INTRODUCTION}

Throughout this paper, we refer that $[A(\cdot), B(\cdot)]$ is a $T$-periodic pair if $A(\cdot) \in L^{\infty}\left(\mathbb{R}^{+} ; \mathbb{R}^{n \times n}\right)$ and $B(\cdot) \in$ $L^{\infty}\left(\mathbb{R}^{+} ; \mathbb{R}^{n \times m}\right)$ satisfy respectively $A(t+T)=A(t)$ for a.e. $t \geq 0$ and $B(t+T)=B(t)$ for a.e. $t \geq 0$. Corresponding to each $T$-periodic pair $[A(\cdot), B(\cdot)]$, we consider the controlled equation

$$
\dot{y}(t)=A(t) y(t)+B(t) u(t), \quad t \geq 0 .
$$

Here, $u(\cdot)$ is a control taken from the space

$$
\mathcal{U}_{\mathrm{ad}} \triangleq L_{\mathrm{loc}}^{2}\left(\mathbb{R}^{+} ; \mathbb{R}^{m}\right), \text { with } \mathbb{R}^{+} \triangleq[0, \infty) .
$$

Keywords and phrases. Linear time-periodic controlled ODEs, periodic stabilization, null-controllable subspaces, the transformation over time $T$.

* This author was partially supported by the National Natural Science Foundation of China under grants 11161130003 and 11171264 and by the National Basis Research Program of China (973 Program) under grant 2011CB808002.

** This author was partially supported by the National Natural Science Foundation of China under grants 10801041 and $1083100 \%$.

1 School of Mathematics and Statistics, Wuhan University, Wuhan, 430072, China. wanggs62@yeah.net

2 School of Mathematical Sciences, Fudan University, KLMNS, Shanghai 200433, China.

Corresponding author: yashanxu@fudan.edu.cn 
For each control $u(\cdot) \in \mathcal{U}_{\text {ad }}$ and each $x \in \mathbb{R}^{n}$, we denote by $y(\cdot ; 0, x, u)$ the solution of equation (1.1) with the initial condition $y(0)=x$. equation (1.1) is said to be $k T$-periodically stabilizable if there exists a $k T$-periodic $K(\cdot)$ in $L^{\infty}\left(\mathbb{R}^{+} ; \mathbb{R}^{m \times n}\right)$ (with $k \in \mathbb{N}$ ) such that the zero solution to the equation

$$
\dot{y}(t)=[A(t)+B(t) K(t)] y(t), \quad t \geq 0
$$

is exponentially stable. Any such a $K(\cdot)$ is called a $k T$-periodic feedback stabilization law for the pair $[A(\cdot), B(\cdot)]$ (or for Eq. (1.1)). The main purposes of this study are (i) to build up two different $T$-periodic stabilization criteria for a $T$-periodic pair; (ii) to construct two different periodic feedback stabilization laws for a $T$-periodically stabilizable pair, one is $n T$-periodic, while another is $T$-periodic.

Two important types of solutions for ordinary differential equations are equilibrium and periodic solutions. A mature theory on stability and stabilization for equilibrium solutions of time-invariant linear ordinary differential equations has been established. One of the most important results on the stability is as follows (see, for instance, [3]): The zero solution to the equation $\dot{y}=A y$ where $A \in \mathbb{R}^{n \times n}$ is exponentially stable if and only if $\sigma(A) \in \mathbb{C}^{-}$(where $\mathbb{C}^{-}$is denoted by the complex half plane $\{z \in \mathbb{C}: \operatorname{Re}(z)<0\}$ ). The most important result about stabilization is the following Kalman's criterion (see, for instance, $[7,20]$ ): A pair of matrices $[A, B]$ in $\mathbb{R}^{n \times n} \times \mathbb{R}^{n \times m}$ is stabilizable (i.e., there exists a matrix $K$ in $\mathbb{R}^{m \times n}$ such that $\sigma(A+B K) \in \mathbb{C}^{-}$) if and only if $\operatorname{rank}(\lambda I-A, B)=n$ for each $\lambda \in \mathbb{C} \backslash \mathbb{C}^{-}$. When a pair $[A, B]$ is stabilizable, any matrix $K$ with the abovementioned property is called a feedback stabilization law for the pair $[A, B]$. The usual structure of such feedback laws is connected with either Riccati equations or Lyapunov functions (see, for instance, either Chap. 7, [16] or Chap. 5, [20]). With respect to linear time-periodic ordinary differential equations, a well-established stability theory has been developed. One of the most important results is the following stability criterion (see, for instance, $[9,10]$ ): any periodic solution $y_{\pi}(\cdot)$ to the equation: $\dot{y}(t)=A(t) y(t), t \geq 0$ (where $A(\cdot) \in L^{\infty}\left(\mathbb{R}^{+} ; \mathbb{R}^{n \times n}\right.$ ) is $T$-periodic) is exponentially stable if and only if $\sigma(\mathcal{P}) \subset \mathcal{B}$. In view of Kalman's criterion for the stabilization of time-invariant pairs and the periodic stability theory mentioned above, it is natural and important to ask for periodic stabilization criteria on a $T$-periodic pair and the way to construct periodic feedback stabilization laws for a periodically stablizable pair.

To present our main results, some preliminaries are given in order. We start with some notations. For each $k \in \mathbb{N},\|\cdot\|_{\mathbb{R}^{k}}$ and $\langle\cdot, \cdot\rangle_{\mathbb{R}^{k}}$ denote accordingly the Euclidean norm and inner product in $\mathbb{R}^{k}$. We will simply write them as $\|\cdot\|$ and $\langle\cdot, \cdot\rangle$, when it will not cause any confusion. We denote by $\left\{e_{1}, \ldots, e_{k}\right\}$ the standard basis of $\mathbb{R}^{k}$, with $k \in \mathbb{N}$, where each $e_{i}$ is a column vector. We treat an element in $\mathbb{R}^{k_{1} \times k_{2}}$, with $k_{1}, k_{2} \in \mathbb{N}$, as a $k_{1} \times k_{2}$ real matrix. When $k_{1}, k_{2} \in \mathbb{N}, 0_{k_{1} \times k_{2}}$ and $I_{k_{1}}$ stand for the $k_{1} \times k_{2}$ null matrix and the $k_{1} \times k_{1}$ identity matrix, respectively. For each matrix $P,\|P\|$ represents its operator norm, $P^{*}$ denotes its transpose and $P^{\sim 1}$ stands for its Moore-Penrose inverse (see [19] for its definition). For each linear map $L$ over a linear space, $\sigma(L), \mathcal{N}(L)$ and $\mathcal{R}(L)$ denote its spectrum, kernel and range, respectively. We use $\mathcal{B}$ to denote the unit open ball in the complex plane $\mathbb{C}, \mathcal{B}^{c}$ to denote the complement of $\mathcal{B}$. Let $\Phi_{A(\cdot)}(\cdot)$ be the fundamental solution associated with $A(\cdot)$. We will simply write it as $\Phi(\cdot)$ when there is no risk to cause any confusion. Let

$$
\mathcal{P} \triangleq \Phi(T),
$$

which is called the transformation over time $T$ associated with $A(\cdot)$ (see p. 256 in [3]).

Next, for each $T$-periodic pair $[A(\cdot), B(\cdot)]$ and $\varepsilon>0$, two linear ordinary differential equations with initial conditions are introduced. The first one is

$$
\dot{S}_{n}(t)-A(t) S_{n}(t)-S_{n}(t) A(t)^{*}+\frac{1}{\varepsilon} B(t) B(t)^{*}=0, \quad t \in[0, n T], \quad S_{n}(n T)=I ;
$$

while the second one is

$$
\dot{S}(t)-A(t) S(t)-S(t) A(t)^{*}+\frac{1}{\varepsilon} B(t) B(t)^{*}=0, \quad t \in[0, T], \quad S(T)=\mathcal{P} X X^{*} \mathcal{P}^{*},
$$

where $X$ is an invertible matrix in $\mathbb{R}^{n \times n}$. 
Then, two periodic matrix-valued functions $K_{n}^{\varepsilon}(\cdot), K^{\varepsilon}(\cdot)$ and a special matrix $\bar{Q}$ will be defined. Write $S_{n}^{\varepsilon}(\cdot)$ and $S^{\varepsilon}(\cdot)$ for the unique solutions of equations (1.5) and (1.6), respectively. It is proved that $S_{n}^{\varepsilon}(\cdot)$ and $S^{\varepsilon}(\cdot)$ are symmetric and positive definite matrix-valued functions over $[0, n T]$ and $[0, T]$, respectively (see Lem. 2.3 and Step 2 in Part 2 of the Proof of Thm. 1.1). It is also shown that

$$
\bar{Q} \triangleq \lim _{\varepsilon \rightarrow 0^{+}}\left(S_{n}^{\varepsilon}(0)\right)^{-1}
$$

is a symmetric and positive semi-definite matrix (see Lem. 2.3). For each $\varepsilon>0$, we define an $n T$-periodic matrix-valued function $K_{n}^{\varepsilon}(\cdot)$ in $L^{\infty}\left(\mathbb{R}^{+} ; \mathbb{R}^{m \times n}\right)$ by

$$
K_{n}^{\varepsilon}(t)=-\frac{1}{\varepsilon} B^{*}(t)\left(S_{n}^{\varepsilon}(t)\right)^{-1} \text { for a.e. } t \in[0, n T) ; K_{n}^{\varepsilon}(t)=K_{n}^{\varepsilon}(t+n T) \text { for a.e. } t \in \mathbb{R}^{+} .
$$

Similarly, a $T$-periodic matrix-valued function $K^{\varepsilon}(\cdot)$ in $L^{\infty}\left(\mathbb{R}^{+} ; \mathbb{R}^{m \times n}\right)$ is defined by

$$
K^{\varepsilon}(t)=-\frac{1}{\varepsilon} B^{*}(t)\left(S^{\varepsilon}(t)\right)^{-1} \text { for a.e. } t \in[0, T) ; K^{\varepsilon}(t)=K^{\varepsilon}(t+T) \text { for a.e. } t \in \mathbb{R}^{+} .
$$

Then, for each $T$-periodic pair $[A(\cdot), B(\cdot)]$, its null-controllable subspace and the null-controllable subspace on $[0, k T]$, with $k \in \mathbb{N}$, are accordingly defined by

$$
V_{[A(\cdot), B(\cdot)]} \triangleq\left\{x \in \mathbb{R}^{n} \mid \exists u \in \mathcal{U}_{\text {ad }} \text { and } t>0 \text { s.t. } y(t ; 0, x, u)=0\right\}
$$

and

$$
V_{[A(\cdot), B(\cdot)], k} \triangleq\left\{x \in \mathbb{R}^{n} \mid \exists u \in \mathcal{U}_{\text {ad }} \text { s.t. } y(k T ; 0, x, u)=0\right\} .
$$

We will simply write them as $V$ and $V_{k}$, respectively, when it will not cause any confusion.

Finally, given a finite dimension linear space $H$ and a linear map $L$ on $H$, there is a unique pair $\left(H_{1}(L), H_{2}(L)\right)$ of subspaces such that (see, for instance, Theorem 1 on p. 78, [10])

$$
H=H_{1}(L) \oplus H_{2}(L),
$$

where $H_{1}(L)$ and $H_{2}(L)$ are invariant under $L$ and satisfy $\sigma\left(\left.L\right|_{H_{1}(L)}\right) \subset \mathcal{B}$ and $\sigma\left(\left.L\right|_{H_{2}(L)}\right) \subset \mathcal{B}^{c}$.

The main results of this paper are presented by the following two theorems.

Theorem 1.1. Let $[A(\cdot), B(\cdot)]$ be a T-periodic pair with $\mathcal{P}$ and $\bar{Q}$ given by (1.4) and (1.7), respectively. Then, the following statements are equivalent:

(a) the pair $[A(\cdot), B(\cdot)]$ is $n T$-periodically stabilizable;

(b) the pair $[A(\cdot), B(\cdot)]$ is T-periodically stabilizable;

(c) it holds that

$$
\sigma\left(\bar{Q}^{\sim 1} \bar{Q} \mathcal{P}\right) \subset \mathcal{B}
$$

Furthermore, when $[A(\cdot), B(\cdot)]$ is T-periodically stabilizable, each $K_{n}^{\varepsilon}(\cdot)$ defined by (1.8), with $\left\|\left(S_{n}^{\varepsilon}(0)\right)^{-1}-\bar{Q}\right\|<1$, is an $n T$-periodic feedback stabilization law for this pair; and meanwhile there are an invertible $X \in \mathbb{R}^{n \times n}$ (depending on $V_{[A(, \cdot), B(\cdot)], k}$, with $k=1, \ldots, n$ ) and a positive number $\varepsilon_{0}$ (depending on $n$, $\|X\|$ and $\|\mathcal{P}\|)$ such that each $K^{\varepsilon}(\cdot)$ given by (1.9), with $\varepsilon \leq \varepsilon_{0}$, is a T-periodic feedback stabilization law for this pair.

Theorem 1.2. Let $[A(\cdot), B(\cdot)]$ be a $T$-periodic pair with $\mathcal{P}, V$ and $\mathbb{R}_{2}^{n}(\mathcal{P})$ given by (1.4), (1.10) and (1.12), respectively. Then, the following statements are equivalent:

(i) the pair $[A(\cdot), B(\cdot)]$ is T-periodically stabilizable;

(ii) it holds that

$$
\mathbb{R}_{2}^{n}(\mathcal{P}) \subseteq V
$$


Based on Theorem 1.1, we can get an estimate on the decay rate for solutions of equation (1.3) where $[A(\cdot), B(\cdot)]$ is $T$-periodically stabilizable and $K(\cdot)=K_{n}^{\varepsilon}(\cdot)$ with $\varepsilon$ sufficiently small. To state it, we let

$$
\bar{\rho}=\min _{\lambda \in \sigma\left(\bar{Q}^{\sim 1} \bar{Q} \mathcal{P}\right) \backslash\{0\}}-\ln |\lambda| / T .
$$

Write $P_{V}$ and $P_{V^{\perp}}$ for the orthogonal projections of $\mathbb{R}^{n}$ to $V$ and $V^{\perp}$, respectively.

Theorem 1.3. Let $[A(\cdot), B(\cdot)]$ be a T-periodically stabilizable pair with $V$ and $K_{n}^{\varepsilon}(\cdot)$ given by (1.10) and (1.8) respectively. Then, given $\delta>0$, there are positive $\varepsilon \triangleq \varepsilon(\delta)$ and $M \triangleq M(\delta)$ such that the solutions $y^{\varepsilon}(\cdot)$ to $(1.3)$ with $K(\cdot)=K_{n}^{\varepsilon}(\cdot)$ satisfy

$$
\left\|y^{\varepsilon}(t)\right\| \leq M\left(\mathrm{e}^{-t / \delta}\left\|P_{V}\left(y^{\varepsilon}(0)\right)\right\|+\mathrm{e}^{-(\bar{\rho}-\delta) t}\left\|P_{V^{\perp}}\left(y^{\varepsilon}(0)\right)\right\|\right) \text { for all } t \in \mathbb{R}^{+} .
$$

Theorem 1.2 has the following application: For each $T$-periodic $A(\cdot) \in L^{\infty}\left(\mathbb{R}^{+} ; \mathbb{R}^{n}\right)$, define

$$
\mathcal{C B}_{A(\cdot)}=\left\{\hat{B} \in \mathbb{R}^{n \times m} \mid m \in \mathbb{N},[A(\cdot), \hat{B}] \text { is T-periodically stabilizable }\right\} .
$$

For each $\hat{B} \in \mathcal{C B}_{A(\cdot)}$, denote by $M(\hat{B})$ the number of columns of $\hat{B}$. Set

$$
M\left(\mathcal{C B}_{A(\cdot)}\right) \triangleq \min \left\{M(\hat{B}) \mid \hat{B} \in \mathcal{C B}_{A(\cdot)}\right\}
$$

With the aid of Theorem 1.2, we can find a way to determine the number $M\left(\mathcal{C B}_{A(\cdot)}\right)$ and to design a matrix $\hat{B}$ in $\mathcal{C B}_{A(\cdot)}$, with $M\left(\mathcal{C B}_{A(\cdot)}\right)$ columns. In particular, when $A(\cdot) \equiv A($ i.e., $A(\cdot)$ is time-invariant), our way leads to

$$
M\left(\mathcal{C B}_{A}\right)=\max _{\lambda \in \sigma(A) \backslash \mathbb{C}^{-}} m(\lambda),
$$

where $m(\lambda)$ denotes the geometric multiplicity of the eigenvalue $\lambda$. Moreover, a corresponding matrix $\hat{B}$ has an explicit expression (see Rem. 6.19).

Several remarks are given in order:

- R. Brockett formulated the following problem in [5]: What are the conditions on a triple $(A, B, C)$ (with $A \in \mathbb{R}^{n \times n}, B \in \mathbb{R}^{n \times m}$ and $C \in \mathbb{R}^{p \times n}$ ) ensuring the existence of a periodic $K(\cdot)$ (with $K(t) \in \mathbb{R}^{m \times p}$ ) such that the system $\dot{y}(t)=A y(t)+B K(t) C y(t)$ is asymptotically stable? After the Brockett problem, it was pointed out in [15] that however, the stabilizaiton of the above system by a constant matrix $K$ is a classical problem in the control theory, from this point of view, the Brockett problem can be reformulated as: can the time periodic matrices $K(t)$ aid in the stabilization? Furthermore, the positive answer for the reformulated Brockett problem (at least for the case where $n=2$ ) was given in [15]. The connections of our Theorem 1.1 and the reformulated Brockett problem are as follows. By Theorem 1.1, we will find that (see Rem. 3.1) when $[A(\cdot), B(\cdot)]=[A, B]$ is time-invariant, (1.1) is $T$-periodically stabilizable for some $T>0$ if and only if (1.1) is $T$-periodically stabilizable for any $T>0$ if and only if (1.1) is feedback stabilizable by a constant matrix. Hence, the time periodic matrices $K(t)$ will not aid in the stabilization of any triple $(A, B, C)$ with $\operatorname{rank} C=n$, i.e., the reformulated Brockett problem has the positive answer only if $\operatorname{rank} C<n$.

We expect that our main results, as well as the application of Theorem 1.2, given in Section 6, can be connected with the original Brockett problem. Unfortunately, we have no any result on it so far, except for the trivial case where $\operatorname{rank} C=n$.

- There have been studies on periodic stabilization criteria for linear periodic systems. In [14], the following criterion was established (see Thm. 2 in [14]): $T$-periodic stabilization $\Leftrightarrow \mathrm{H}$-stabilization. Here, a $T$-periodic pair $[A(\cdot), B(\cdot)]$ is said to be $H$-stabilizable, if for each $\lambda \in \sigma(\mathcal{P})$ with $|\lambda| \geq 1$, it holds that

$$
\eta=0 \text {, when } \mathcal{P}^{*} \eta=\lambda \eta \text { and } B^{*}(t)\left(\Phi(t)^{*}\right)^{-1} \eta=0 \text { for a.e. } t \in[0, T] \text {. }
$$


The property (1.19) is a kind of unique continuation property for eigenfunctions of $\mathcal{P}^{*}$ corresponding to $\lambda \in \sigma(\mathcal{P})$ with $|\lambda| \geq 1$. There is a similar version of this kind of unique continuation property in infinite dimensional systems (see [4]). With the aid of the above-mentioned criterion, the authors of [14] built up a $T$-periodic feedback stabilization law via the solution of the following $T$-periodic matrix Riccati equation

$$
\dot{Q}+A^{*} Q+Q A+H^{*} H-Q B B^{*} Q=0 ; Q(t)=Q(t+T), \quad t \in \mathbb{R}^{+},
$$

where $H(\cdot)$ is a $T$-periodic matrix-valued function such that $\left[A(\cdot)^{*}, H(\cdot)^{*}\right]$ is $H$-stabilizable. It was proved in [13] that when both $[A(\cdot), B(\cdot)]$ and $\left[A(\cdot)^{*}, H(\cdot)^{*}\right]$ are $H$-stabilizable, the equation (1.20) admits a unique positive semi-definite matrix-valued $T$-periodic solution (see [13]). Furthermore, $K(\cdot)=-B(\cdot)^{*} Q(\cdot)$ is a $T$-periodical feedback stabilization law.

The novelties of our paper, compared with the results in [14], are as follows: $(a)$ we establish two criterions differing from the $\mathrm{H}$-stabilization in [14]. (b) We build up two kinds of periodic stabilization laws through solving accordingly two linear equations with initial conditions.

- The study in [14] was partially motivated by [6], where the author proved the fact that a $T$-periodic pair $[A(\cdot), B(\cdot)]$ is controllable if and only if it is controllable over $[0, n T]$. This also hints us to define the concept of $n T$-periodic stabilization and to build up a $n T$-periodic stabilization law.

- The equivalent condition (1.13) in Theorem 1.1 is a natural extension of Kalman's rank condition (see Rem. 3.1).

- The matrix $X$ in Theorem 1.1 can be explicitly structured. (See Step 1 in Part 2 of the proof of Thm. 1.1).

- When $[A(\cdot), B(\cdot)]$ is $T$-periodically stabilizable, one can use a very similar method as that used in the Proof of Theorem 1.3 to derive a similar estimate to (1.16) for solutions $y^{\varepsilon}(\cdot)$ to equation (1.3) with $K(\cdot)=K^{\varepsilon}(\cdot)$ where $K^{\varepsilon}(\cdot)$ is defined by (1.9) with $X$ given by Theorem 1.1. We omit its proof in this paper.

- By our understanding, the procedure to stabilize periodically a system: $\dot{y}(t)=A(t) y(t)$ (where $A(\cdot) \in$ $L^{\infty}\left(\mathbb{R}^{+} ; \mathbb{R}^{n \times n}\right)$ is $T$-periodic) is as follows: one first builds up a $T$-periodic $B(\cdot) \in L^{\infty}\left(\mathbb{R}^{+} ; \mathbb{R}^{n \times m}\right)$ such that $[A(\cdot), B(\cdot)]$ is $T$-periodically stablizable, and then design a periodic (such as $T$-periodic or $n T$-period) $K(\cdot) \in L^{\infty}\left(\mathbb{R}^{+} ; \mathbb{R}^{m \times n}\right)$ such that $A(\cdot)+B(\cdot) K(\cdot)$ is exponentially stable. We call the aforementioned $B(\cdot)$ as a control machine and the corresponding $K(\cdot)$ as a feedback law. Control machines could be treated as control equipments which belong to the category of hardware, while feedback laws could be treated as control programs which belong to the category of software. Thus, it is interesting to answer to following question: How to design a simple $T$-periodic $B(\cdot)$ for a given $T$-periodic $A(\cdot)$ such that $[A(\cdot), B(\cdot)]$ is $T$ periodically stablizable? A matrix $\hat{B}$, with $M\left(\mathcal{C B}_{A(\cdot)}\right)$ columns, in $\mathcal{C B}_{A(\cdot)}$ (see (1.17) and (1.18)) could be one of the simplest ones. From this point of view, we give an answer for the above question, with the help of Theorem 1.2.

- As a byproduct of this study, it is built up that $V=\mathcal{N}(\bar{Q})$ for any $T$-periodic pair $[A(\cdot), B(\cdot)]$, where $V$ is defined by (1.10) and $\bar{Q}$ is defined by (1.7) (see Lem. 2.3). This is a connection between the null-controllable subspace $V$ of a $T$-periodic pair $[A(\cdot), B(\cdot)]$ and the corresponding operator (or matrix) $\bar{Q}$. This connection appears to be new and plays an important role in the proof of our main results.

The rest of this paper is organized as follows: Section 2 presents some preliminary lemmas. Section 3 proves Theorem 1.1. Section 4 verifies Theorems 1.2 and 1.3. Section 5 gives an example to illustrate our main results. Section 6 provides an application of Theorem 1.2, i.e., a way to determine $M\left(\mathcal{C B}_{A(\cdot)}\right)$ given by (1.18).

\section{Preliminary Lemmas}

Lemma 2.1. Let $[A(\cdot), B(\cdot)]$ be a $T$-periodic pair with $\mathcal{P}, V$ and $V_{k}$ given by (1.4), (1.10) and (1.11), respectively. Then

$$
V_{k}=\sum_{j=0}^{k-1} \mathcal{P}^{-j} V_{1} \text { for all } k \in \mathbb{N} ; \quad V=V_{n}
$$


Moreover, it holds that

$$
\mathcal{P} V=V=\mathcal{P}^{-1} V ; \quad \mathcal{P}^{*} V^{\perp}=V^{\perp}=\left(\mathcal{P}^{*}\right)^{-1} V^{\perp} .
$$

Proof. Recall the definition of $V_{k}$ (see (1.11)). It holds that

$$
V_{k}=\left\{\int_{0}^{k T} \Phi^{-1}(s) B(s) u(s) \mathrm{d} s \mid u(\cdot) \in \mathcal{U}_{\mathrm{ad}}\right\} .
$$

Now, we prove (2.1) and (2.2) by three steps as follows:

Step 1. To show the first equality in (2.1)

Arbitrarily fix a $k \in \mathbb{N}$. By the periodicity of $B(\cdot)$ and $\Phi(\cdot)$, we have

$$
\begin{aligned}
& \int_{0}^{k T} \Phi^{-1}(s) B(s) u(s) \mathrm{d} s=\sum_{j=0}^{k-1} \int_{j T}^{(j+1) T} \Phi^{-1}(s) B(s) u(s) \mathrm{d} s \\
& =\sum_{j=0}^{k-1} \int_{0}^{T} \mathcal{P}^{-j} \Phi^{-1}(s) B(s) u(s) \mathrm{d} s \in \sum_{j=0}^{k-1} \mathcal{P}^{-j} V_{1},
\end{aligned}
$$

which leads to $V_{k} \subseteq \sum_{j=0}^{k-1} \mathcal{P}^{-j} V_{1}$. On the other hand, given $x_{1}, \ldots, x_{k} \in V_{1}$, there are $u_{1}(\cdot), \ldots, u_{k}(\cdot) \in \mathcal{U}_{\text {ad }}$ such that

$$
x_{j}=\int_{0}^{T} \Phi(s) B(s) u_{j}(s) \mathrm{d} s, \quad j \in\{1,2, \ldots, k\} .
$$

Let $\hat{x}=\sum_{j=0}^{k-1} \mathcal{P}^{-j} x_{j}$ and define $\hat{u} \in \mathcal{U}_{\mathrm{ad}}$ by

$$
\hat{u}(j T+t)=u_{j}(t) \text { for all } j \in\{1, \ldots, k\}, t \in[0, T), \quad \hat{u}(t)=0 \text { for all } t \in[k T, \infty) .
$$

Then, it holds that

$$
\hat{x}=\int_{0}^{k T} \Phi^{-1}(s) B(s) \hat{u}(s) \mathrm{d} s,
$$

which leads to $\hat{x} \in V_{k}$. From this, $\sum_{j=0}^{k-1} \mathcal{P}^{-j} V_{1} \subseteq V_{k}$. Hence the first equality in (2.1) holds for all $k \in \mathbb{N}$.

Step 2. To prove that $V=V_{n}$

It is obvious that $V_{n} \subseteq V$. To show the reverse, let $x \in V$. By the definition of $V$, there are $u(\cdot) \in \mathcal{U}_{\text {ad }}$ and $t>0$ such that $y(t ; 0, x, u)=0$. Let $\hat{u}(s)=\chi_{(0, t)}(s) u(s)$ for $s \geq 0$ and $N(t)$ the non-negative integer such that $N(t) T<t \leq(N(t)+1) T$. Then

$$
y((N(t)+1) T ; 0, x, \hat{u})=y((N(t)+1) T ; t, 0, \hat{u})=0,
$$

from which, it follows that $x \in V_{N(t)+1}$. On the other hand, it follows from the Hamilton-Cayley theorem and the first equality in (2.1), indicates that

$$
V_{N(t)+1}=\sum_{j=0}^{N(t)} \mathcal{P}^{-j} V_{1} \subseteq \sum_{j=0}^{n-1} \mathcal{P}^{-j} V_{1}=V_{n} .
$$

Therefore, $x \in V_{n}$ which leads to $V=V_{n}$. 
Step 3. To show (2.2).

Since

$$
\mathcal{P}^{-1} V=\mathcal{P}^{-1} V_{n}=\mathcal{P}^{-1} \sum_{j=0}^{n-1} \mathcal{P}^{-j} V_{1}=\sum_{j=1}^{n} \mathcal{P}^{-j} V_{1} \subseteq \sum_{j=0}^{n-1} \mathcal{P}^{-j} V_{1}=V_{n},
$$

it follows from the second equality in (2.1) that

$$
\mathcal{P}^{-1} V \subset V \text {. }
$$

Because $\mathcal{P}$ is invertible, $\operatorname{dim}\left(\mathcal{P}^{-1} V\right)=\operatorname{dim} V$, which, along with (2.4), yields that $\mathcal{P}^{-1} V=V$, i.e., $V=\mathcal{P} V$. Hence, the first statement in (2.2) stands. The second statement in (2.2) is a direct consequence of the first one. This completes the proof.

Define, for each $\varepsilon>0, x \in \mathbb{R}^{n}$ and $t \in[0, n T)$, the linear quadratic optimal control problem

$$
(L Q)_{t, x}^{\varepsilon}: \inf _{u \in L^{2}\left(t, n T ; \mathbb{R}^{m}\right)} J^{\varepsilon}(u(\cdot) ; t, x) .
$$

Here,

$$
J^{\varepsilon}(u(\cdot) ; t, x)=\int_{t}^{n T} \varepsilon<u(s), u(s)>_{\mathbb{R}^{m}} \mathrm{~d} s+<y(n T ; t, x, u), y(n T ; t, x, u)>_{\mathbb{R}^{n}},
$$

where $y(\cdot ; t, x, u)$ is the solution to equation $(1.1)$ over $[t, n T]$, with the initial condition $y(t)=x$. The corresponding value function is

$$
W^{\varepsilon}(t, x)=\inf _{u \in L^{2}\left(t, n T ; \mathbb{R}^{m}\right)} J^{\varepsilon}(u(\cdot) ; t, x), \quad t \in[0, n T) \text { and } x \in \mathbb{R}^{n} .
$$

The classical theory on optimal controls (see, for instance, Thm. 37, p. 364, [20]) leads to the next lemma.

Lemma 2.2. For each $\varepsilon>0$, it holds that

$$
W^{\varepsilon}(t, x)=\left\langle x, Q_{n}^{\varepsilon}(t) x\right\rangle \text { for all }(t, x) \in[0, n T) \times \mathbb{R}^{n},
$$

where $Q_{n}^{\varepsilon}(\cdot)$ is a symmetric and positive definite $n \times n$ matrix-valued function (over $[0, n T]$ ) solving uniquely the Riccati equation

$$
\dot{Q}(t)+A(t)^{*} Q(t)+Q(t) A(t)-\frac{1}{\varepsilon} Q(t) B(t) B(t)^{*} Q(t)^{*}=0, t \in[0, n T] ; \quad Q(n T)=I .
$$

Moreover, if $y^{t, x}(\cdot)$ is the solution to the equation

$$
\dot{y}(s)=\left(A(s)+B(s)\left(-\frac{1}{\varepsilon} B(s)^{*} Q_{n}^{\varepsilon}(s)\right)\right) y(s), \quad s \geq t ; \quad y(t)=x,
$$

then the function

$$
\bar{u}_{x}^{\varepsilon}(s)=-\frac{1}{\varepsilon} B(s)^{*} Q_{n}^{\varepsilon}(s) y^{t, x}(s) \text { for a.e. } s \in[t, n T]
$$

is the unique optimal control to Problem $(L Q)_{t, x}^{\varepsilon}$.

Lemma 2.3. Let $[A(\cdot), B(\cdot)]$ be a $T$-periodic pair with $V, Q_{n}^{\varepsilon}(\cdot)$ and $\bar{Q}$ given by (1.10), (2.6) and (1.7) respectively. Then the following statements stand:

(i) the solution $S_{n}^{\varepsilon}(\cdot)$ of equation (1.5) is $Q_{n}^{\varepsilon}(\cdot)^{-1}$;

(ii) the matrix $\bar{Q}$ is well defined, and is symmetric and positive semi-definite; 
(iii) it holds that

$$
V=\mathcal{N}(\bar{Q}) \text { and } V^{\perp}=\mathcal{R}(\bar{Q}) .
$$

Proof.

(i) Since $Q_{n}^{\varepsilon}(\cdot)$ solves equation (2.6), it follows from some simple calculation that $S_{n}^{\varepsilon}(\cdot)$ is the solution to equation (1.5).

(ii) Arbitrarily fix $x \in \mathbb{R}^{n}$ and $u(\cdot) \in L^{2}\left(0, n T ; \mathbb{R}^{m}\right)$. Clearly, $J^{\varepsilon_{1}}(u ; 0, x) \leq J^{\varepsilon_{2}}(u ; 0, x)$ when $\varepsilon_{1} \leq \varepsilon_{2}$. Thus, $\left\langle x, Q_{n}^{\varepsilon}(0) x\right\rangle$ (which equals to $W^{\varepsilon}(0, x)$ by Lem. 2.2) is monotonically increasing with respect to $\varepsilon$ and bounded from below by 0 . Hence, $\lim _{\varepsilon \rightarrow 0+}\left\langle x, Q_{n}^{\varepsilon}(0) x\right\rangle$ exists for each $x \in \mathbb{R}^{n}$. From this, $\lim _{\varepsilon \rightarrow 0+} Q_{n}^{\varepsilon}(0)$ exists, since we are working in the finite dimensional space $\mathbb{R}^{n}$. Clearly, this limit is a symmetric and positive semi-definite matrix, since $Q_{n}^{\varepsilon}(0)$ is symmetric and positive definite for each $\varepsilon>0$.

(iii) We first claim the statement that

$$
x \in V \text { if and only if }\langle x, \bar{Q} x\rangle=0 .
$$

In fact, on one hand, if $x \in V$, then it follows from Lemma 2.1 that $x \in V_{n}$, i.e., there is a control $\hat{u}(\cdot) \in \mathcal{U}_{\mathrm{ad}}$ such that $y(n T ; 0, x, \hat{u})=0$. We restrict this control on $[0, n T]$ and still denoted by $\hat{u}(\cdot)$ the restricted control, which is clearly in $L^{2}\left(0, n T ; \mathbb{R}^{m}\right)$. Then, one can easily check that

$$
0 \leq\langle x, \bar{Q} x\rangle=\lim _{\varepsilon \rightarrow 0+} W^{\varepsilon}(0, x) \leq \lim _{\varepsilon \rightarrow 0+} J^{\varepsilon}(\hat{u} ; 0, x)=\lim _{\varepsilon \rightarrow 0+} \varepsilon \int_{0}^{n T}\|\hat{u}(t)\|^{2} \mathrm{~d} t=0 .
$$

On the other hand, if $x \in \mathbb{R}^{n}$ satisfies $\langle x, \bar{Q} x\rangle=0$, then by the definition of $J^{\varepsilon}$,

$$
0=\langle x, \bar{Q} x\rangle=\lim _{\varepsilon \rightarrow 0+u \in L^{2}\left(0, n T ; \mathbb{R}^{m}\right)} J^{\varepsilon}(u ; 0, x) \geq \inf _{u \in L^{2}\left(0, n T ; \mathbb{R}^{m}\right)}\|y(n T ; 0, x, u)\|^{2} .
$$

Since

$$
\left\{y(n T ; 0, x, u) \mid u \in L^{2}\left(0, n T ; \mathbb{R}^{m}\right)\right\}=\Phi(n T) x+\Phi(n T) V,
$$

(where $\Phi(\cdot)$ denotes the fundamental solution associated with $A(\cdot)$ ), it follows from (2.11) that

$$
\inf _{z \in \Phi(n T) x+\Phi(n T) V}\|z\|^{2}=0 .
$$

From this, $\Phi(n T) x+\Phi(n T) x_{0}=0$ for some $x_{0} \in V$, which leads to $x \in V$, and proves (2.10).

Finally, since $\bar{Q}$ is symmetric and positive semi-definite, it is clear that $\langle x, \bar{Q} x\rangle=0$ if and only if $\bar{Q} x=0$. This, along with (2.10), leads to the first equality in (2.9). The second equality in (2.9) follows from facts that $V^{\perp}=(\mathcal{N}(\bar{Q}))^{\perp}=\mathcal{R}\left(\bar{Q}^{*}\right)=\mathcal{R}(\bar{Q})$. This completes the proof.

The next lemma is a modified version of Lemma 1 in [11].

Lemma 2.4. Let $[A(\cdot), B(\cdot)]$ be a $T$-periodic pair with $V, \mathcal{P}$ and $\bar{Q}$ given by (1.10), (1.4) and (1.7), respectively. Then there are $T$-periodic $A_{11}(\cdot), A_{12}(\cdot)$ and $A_{22}(\cdot)$ in $L^{\infty}\left(\mathbb{R}^{+} ; \mathbb{R}^{n \times n}\right)$ and $B_{1}(\cdot)$ in $L^{\infty}\left(\mathbb{R}^{+} ; \mathbb{R}^{n \times m}\right)$ such that any solution $y(\cdot)$ of equation $(1.1)$, with a control $u(\cdot) \in \mathcal{U}_{\mathrm{ad}}$, can be uniquely expressed as

$$
y(t)=y_{1}(t)+y_{2}(t), \quad t \in \mathbb{R}^{+},
$$

where the pair $\left(y_{1}(\cdot), y_{2}(\cdot)\right)$ satisfies

$$
\begin{aligned}
& \left\{\begin{array}{l}
\dot{y_{1}(t)}=A_{11}(t) y_{1}(t)+A_{12}(t) y_{2}(t)+B_{1}(t) u(t), \\
\dot{y_{2}}(t)=\quad A_{22}(t) y_{2}(t),
\end{array}\right. \\
& y_{1}(t) \in \Phi(t) V \text { and } y_{2}(t) \in\left(\Phi(t)^{-1}\right)^{*} V^{\perp} \text { for each } t \in \mathbb{R}^{+},
\end{aligned}
$$

and

$$
y_{2}(n T)=\bar{Q}^{\sim 1} \bar{Q} \mathcal{P}^{n} y_{2}(0) \in V^{\perp} .
$$

Here, $\Phi(\cdot)$ denotes the fundamental solution associated with $A(\cdot)$. 
Proof. By Lemma 1 in [11], there are $\hat{A}_{11}(\cdot), \hat{A}_{12}(\cdot)$ and $\hat{A}_{22}(\cdot)$ in $L^{\infty}\left(\mathbb{R}^{+} ; \mathbb{R}^{n \times n}\right)$ and $\hat{B}_{1}(\cdot)$ in $L^{\infty}\left(\mathbb{R}^{+} ; \mathbb{R}^{n \times m}\right)$, which depend only on $[A(\cdot), B(\cdot)]$, such that any solution $y(\cdot)$ to equation (1.1), with a control $u(\cdot)$, can be uniquely expressed by $(2.12)$, where $\left(y_{1}(\cdot), y_{2}(\cdot)\right)$ satisfies

$$
\begin{cases}\dot{y}_{1}(t)=\hat{A}_{11}(t) y_{1}(t)+ & \hat{A}_{12}(t) y_{2}(t)+\hat{B}_{1}(t) u(t), \quad t \in \mathbb{R}^{+} \\ \dot{y}_{2}(t)= & \hat{A}_{22}(t) y_{2}(t),\end{cases}
$$

and (2.14) (see Rem. 2.5 following the proof of this lemma). Define $A_{11}(\cdot), A_{12}(\cdot), A_{22}(\cdot)$ and $B_{1}(\cdot)$ by

$$
\begin{aligned}
& A_{11}(k T+t)=\hat{A}_{11}(t), \quad A_{12}(k T+t)=\hat{A}_{12}(t), \\
& A_{22}(k T+t)=\hat{A}_{22}(t), \quad B_{1}(k T+t)=\hat{B}_{1}(t),
\end{aligned} \quad t \in[0, T), k \in \mathbb{N} .
$$

Clearly, they are $T$-periodic.

Now we arbitrarily fix a control $u(\cdot) \in \mathcal{U}_{\text {ad }}$ and let $y(\cdot)$ be a solution to equation (1.1) corresponding to this control $u(\cdot)$. For each $k \in \mathbb{N}$, we define

$$
y^{k}(t)=y(t+k T) \quad \text { and } u^{k}(t)=u(t+k T), \quad t \geq 0 .
$$

From the $T$-periodicity of $[A(\cdot), B(\cdot)]$ and $(2.18)$, one can easily check that each $y^{k}(\cdot)$ is a solution to equation (1.1) corresponding to the control $u^{k}(\cdot)$. Then, by Lemma 1 in [11], $y^{k}(\cdot)$ can be uniquely expressed as

$$
y^{k}(t)=y_{1}^{k}(t)+y_{2}^{k}(t), \quad t \in \mathbb{R}^{+},
$$

where $\left(y_{1}^{k}(\cdot), y_{2}^{k}(\cdot)\right)$ satisfies

$$
\left\{\begin{array}{ll}
\frac{\mathrm{d}}{\mathrm{d} t} y_{1}^{k}(t)=\hat{A}_{11}(t) y_{1}^{k}(t)+\hat{A}_{12}(t) y_{2}^{k}(t)+\hat{B}_{1}(t) u^{k}(t), & \\
\frac{\mathrm{d}}{\mathrm{d} t} y_{2}^{k}(t)= & \hat{A}_{22}(t) y_{2}^{k}(t),
\end{array} \quad t \in \mathbb{R}^{+}\right.
$$

and

$$
y_{1}^{k}(t) \in \Phi(t) V \text { and } y_{2}^{k}(t) \in\left(\Phi(t)^{-1}\right)^{*} V^{\perp} \text { for all } t \in \mathbb{R}^{+} .
$$

From (2.19), (2.18), (2.12), (2.14) and (2.2), three observations are in order:

$$
\begin{aligned}
& y_{1}^{k}(t)+y_{2}^{k}(t)=y^{k}(t)=y(t+k T)=y_{1}(t+k T)+y_{2}(t+k T), k \in \mathbb{N}, t \in[0, T) ; \\
& y_{1}(t+k T) \in \Phi(t+k T) V=\Phi(t) \Phi(k T) V=\Phi(t) \Phi(T)^{k} V=\Phi(t) V, k \in \mathbb{N}, t \in[0, T) ; \\
& y_{2}(t+k T) \in\left(\Phi(t+k T)^{-1}\right)^{*} V^{\perp}=\left(\Phi(t)^{-1}\right)^{*} V^{\perp}, k \in \mathbb{N}, t \in[0, T) .
\end{aligned}
$$

These, along with (2.21), indicates that

$$
y_{1}^{k}(t)=y_{1}(t+k T) \text { and } y_{2}^{k}(t)=y_{2}(t+k T) \text { for all } t \geq 0 \text { and } k \in \mathbb{N} .
$$

From these, $(2.16),(2.20)$ and $(2.18)$, one can easily check that $\left(y_{1}(\cdot), y_{2}(\cdot), u(\cdot)\right)$ satisfies system $(2.13)$ with $T$-periodic $A_{11}(\cdot), A_{12}(\cdot), A_{22}(\cdot)$ and $B_{1}(\cdot)$ given by $(2.17)$.

The rest is to show (2.15). By the variation of constant formula and (1.4),

$$
y(n T)=\mathcal{P}^{n} y(0)+\mathcal{P}^{n} \int_{0}^{n T} \Phi^{-1}(s) B(s) u(s) \mathrm{d} s .
$$


On one hand, it follows from (2.12), (2.14) and (1.4) that

$$
y(n T)=y_{1}(n T)+y_{2}(n T), \quad y_{1}(n T) \in \mathcal{P}^{n} V \text { and } y_{2}(n T) \in\left(\mathcal{P}^{-n}\right)^{*} V^{\perp} .
$$

Because $\mathcal{P} V=V$ and $\left(\mathcal{P}^{-1}\right)^{*} V^{\perp}=V^{\perp}$ (see (2.2) in Lemma 2.1), it holds that $y_{1}(n T) \in V, y_{2}(n T) \in V^{\perp}$. Hence,

$$
P_{V^{\perp}}(y(n T))=y_{2}(n T) .
$$

On the other hand, since $y(0)=y_{1}(0)+y_{2}(0), y_{1}(0) \in V$ and $y_{2}(0) \in V^{\perp}$, it holds that

$$
\mathcal{P}^{n} y(0)=\mathcal{P}^{n} y_{1}(0)+\mathcal{P}^{n} y_{2}(0) \text { and } \mathcal{P}^{n} y_{1}(0) \in \mathcal{P}^{n} V=V .
$$

By Lemma 2.6 (where $M=\bar{Q}$ ), we see that

$$
\begin{gathered}
\mathcal{P}^{n} y_{2}(0)=\bar{Q}^{\sim 1} \bar{Q} \mathcal{P}^{n} y_{2}(0)+\left(\mathcal{P}^{n} y_{2}(0)-\bar{Q}^{\sim 1} \bar{Q} \mathcal{P}^{n} y_{2}(0)\right), \\
\bar{Q}^{\sim 1} \bar{Q} \mathcal{P}^{n} y_{2}(0) \in \mathcal{R}(\bar{Q})=V^{\perp} \text { and } \mathcal{P}^{n} x-\bar{Q}^{\sim 1} \bar{Q} \mathcal{P}^{n} y_{2}(0) \in \mathcal{N}(\bar{Q})=V .
\end{gathered}
$$

Besides, it follows from Lemma 2.1 that

$$
\mathcal{P}^{n} \int_{0}^{n T} \Phi^{-1}(s) B(s) u(s) d s \in \mathcal{P}^{n} V_{n}=\mathcal{P}^{n} V=V .
$$

Along with (2.22), (2.24), (2.25) and (2.26), this indicates that

$$
P_{V^{\perp}}(y(n T))=P_{V^{\perp}}\left(\mathcal{P}^{n} y(0)\right)=P_{V^{\perp}}\left(\mathcal{P}^{n} y_{2}(0)\right)=\bar{Q}^{\sim 1} \bar{Q} \mathcal{P}^{n} y_{2}(0) .
$$

This, along with (2.23), leads to (2.15).

Finally, the uniqueness of such a decomposition follows from (2.14) at once.

\section{Remark 2.5.}

(i) In [11], the proof of (2.14) is hidden in the proof Lemma 1 (see Line 6, p. 721, [11]), where the null-controllable subspace w.r.t. the time-varying system is defined in the same manner as (1.10).

(ii) In Lemma 1 of [11], it is assumed that $V$ is a proper subspace of $\mathbb{R}^{n}$. This assumption can be dropped if one regards $\{0\}$ as a zero-dimensional subspace of $\mathbb{R}^{n}$.

The next lemma is about a decomposition of vectors related to the Moore-Penrose inverse of a symmetric matrix.

Lemma 2.6. Let $M$ be a symmetric matrix in $R^{n \times n}$. Then, any vector $\xi$ in $\mathbb{R}^{n}$ can be decomposed into two orthogonal vectors $M^{\sim 1} M \xi$ and $\xi-M^{\sim 1} M \xi$ such that

$$
M^{\sim 1} M \xi \in \mathcal{R}(M) \text { and } \xi-M^{\sim 1} M \xi \in \mathcal{N}(M) .
$$

Proof. By the definition of the Moore-Penrose inverse matrix (see [19]) and by the symmetry of $M$, it holds that

$$
M^{\sim 1} M \xi=\left(M^{\sim 1} M\right) \xi=\left(M M^{\sim 1}\right) \xi=M\left(M^{\sim 1} \xi\right) \in \mathcal{R}(M) ;
$$

and

$$
M\left(\xi-M^{\sim 1} M \xi\right)=M \xi-\left(M M^{\sim 1} M\right) \xi=M \xi-M \xi=0 .
$$

These lead to (2.27). Besides, it follows from the symmetry of $M$ that $\mathcal{R}(M)=\mathcal{N}\left(M^{*}\right)^{\perp}=\mathcal{N}(M)^{\perp}$. Hence, the vectors $M^{\sim 1} M \xi$ and $\left(\xi-M^{\sim 1} M \xi\right)$ are orthogonal.

The next lemma is a direct consequence of Theorem 1 on page 67 in [10]. 
Lemma 2.7. Let $H$ be a real $n$-dimensional linear space. Let $L$ be a linear map on $H$. Then $H$ can be uniquely decomposed as

$$
H=H_{1}(L) \bigoplus H_{2}(L),
$$

where $H_{1}(L)$ and $H_{2}(L)$ are invariant under $L$ and satisfy accordingly

$$
\sigma\left(\left.L\right|_{H_{1}(L)}\right) \subset \mathcal{B} \text { and } \sigma\left(\left.L\right|_{H_{2}(L)}\right) \subset \mathcal{B}^{c} .
$$

\section{Remark 2.8.}

(i) Let $L$ be a linear map on $\mathbb{R}^{n}$. Let $Z \subset \mathbb{R}^{n}$ be an invariant subspace of $L$. Then, Lemma 2.7 provides a unique decomposition of $Z$ corresponding to the map $\left.L\right|_{Z}$. We will simply denote this decomposition by $\left(Z_{1}(L), Z_{2}(L)\right)$, when there is no risk to cause any confusion.

(ii) Let $[A(\cdot), B(\cdot)]$ be a $T$-periodic pair. Let $V$ be its null controllable subspace. By Lemma 2.2, $V$ is an invariant subspace of $\mathcal{P}$, where $\mathcal{P}$ is the transformation over time $T$ associated with $A(\cdot)$. Thus, Lemma 2.7 provides a unique decomposition $\left(V_{1}(\mathcal{P}), V_{2}(\mathcal{P})\right)$ for the space $V$.

Lemma 2.9. Let $Z$ be a finite-dimensional vector space and $L$ be a linear map on $Z$. Suppose that $Y \subseteq Z$ is an invariant subspace of $L$. Then

$$
Y_{1}(L) \subseteq Z_{1}(L) \quad \text { and } \quad Y_{2}(L) \subseteq Z_{2}(L) .
$$

Proof. Let $\hat{Z}=Y_{1}(L) \oplus Z_{2}(L)$. Then

$$
Z=\hat{Z}+Z=\left(Y_{1}(L)+Z_{1}(L)\right)+Z_{2}(L) .
$$

Subspaces $Y_{1}(L)$ and $Z_{1}(L)$ are invariant under $L$, so is $Y_{1}(L)+Z_{1}(L)$. Clearly, it holds that

$$
\sigma\left(\left.L\right|_{Y_{1}(L)+Z_{1}(L)}\right) \subset \mathcal{B} .
$$

This, together with the fact that $\sigma\left(\left.L\right|_{Z_{2}(L)}\right) \subset \mathcal{B}^{c}$, implies

$$
\left(Y_{1}(L)+Z_{1}(L)\right) \bigcap Z_{2}(L)=\{0\} .
$$

Along with (2.31), this yields

$$
Z=\left(Y_{1}(L)+Z_{1}(L)\right) \bigoplus Z_{2}(L)
$$

Then, because of the uniqueness of the decomposition provided by Lemma 2.7, we see that

$$
Y_{1}(L)+Z_{1}(L)=Z_{1}(L)
$$

which implies that $Y_{1}(L) \subseteq Z_{1}(L)$.

Similarly, we can verify the second conclusion in (2.30). This completes the proof.

We end this section with introducing some notations which will be used in the Proof of Theorems 1.1 and 1.2. By $(2.2), V$ is invariant space of $\mathcal{P}$. Hence, $V_{1}(\mathcal{P})$ and $V_{2}(\mathcal{P})$ are uniquely provided by Lemma 2.7 . We write

$$
k_{1} \triangleq \operatorname{dim} V, \quad k_{2} \triangleq \operatorname{dim} V_{1}(\mathcal{P}), \quad k_{3} \triangleq \operatorname{dim} \mathbb{R}_{1}^{n}(\mathcal{P}) .
$$

Clearly,

$$
\begin{gathered}
k_{1} \geq k_{2} \leq k_{3}, \\
k_{1}-k_{2}=\operatorname{dim} V_{2}(\mathcal{P}), \quad n-k_{3}=\operatorname{dim} \mathbb{R}_{2}^{n}(\mathcal{P}) .
\end{gathered}
$$

Since $V_{2}(\mathcal{P}) \subseteq \mathbb{R}_{2}^{n}(\mathcal{P})$ (see Lem. 2.9), it holds that

$$
k_{1}+k_{3} \leq n+k_{2} .
$$




\section{The Proof of Theorem 1.1}

The proof will be organized as two parts as follows.

Part 1. To show $(a) \Leftrightarrow(c)$

It is hidden in the proof of this part that if $(c)$ stands, then $K_{n}^{\varepsilon}(\cdot)$ given by (1.8) is an $n T$-periodic feedback law when $\varepsilon$ is sufficiently small. The strategy to prove the equivalence in this part is showing

$$
(a) \Leftrightarrow \sigma\left(\bar{Q}^{\sim 1} \bar{Q} \mathcal{P}^{n}\right) \subset \mathcal{B} \text { and }(c) \Leftrightarrow \sigma\left(\bar{Q}^{\sim 1} \bar{Q} \mathcal{P}^{n}\right) \subset \mathcal{B} .
$$

The proof of these two equivalence will be carried by three steps as follows:

Step 1 in Part 1: To prove $(a) \Rightarrow \sigma\left(\bar{Q}^{\sim 1} \bar{Q} \mathcal{P}^{n}\right) \subset \mathcal{B}$

Let $A_{11}(\cdot), A_{12}(\cdot), A_{22}(\cdot)$ and $B_{1}(\cdot)$ be the matrices given by Lemma 2.4. According to Lemma 2.4, each solution $y(\cdot ; 0, x, u)$ to equation (1.1) has a unique decomposition $\left(y_{1}^{x, u}(\cdot), y_{2}^{x, u}(\cdot)\right)$ satisfying $(2.12),(2.13),(2.14)$ and (2.15). By the second equation in (2.13),

$$
y_{2}^{x, u}(t)=y_{2}^{x, 0}(t) \text {, when } t \geq 0, u \in \mathcal{U}_{\mathrm{ad}} .
$$

We claim

$$
\sigma\left(\bar{Q}^{\sim 1} \bar{Q} \mathcal{P}^{n}\right) \subset \mathcal{B} \Leftrightarrow \lim _{t \rightarrow \infty} y_{2}^{x, 0}(t)=0 \text { for all } x \in \mathbb{R}^{n} .
$$

To this end, it suffices to show that

$$
\lim _{t \rightarrow \infty} y_{2}^{x, 0}(t)=0 \forall x \in \mathbb{R}^{n} \Leftrightarrow \lim _{k \rightarrow \infty}\left(\bar{Q}^{\sim 1} \bar{Q} \mathcal{P}^{n}\right)^{k} z=0 \forall z \in V^{\perp}
$$

and

$$
\lim _{k \rightarrow \infty}\left(\bar{Q}^{\sim 1} \bar{Q} \mathcal{P}^{n}\right)^{k} z=0 \forall z \in V^{\perp} \Leftrightarrow \sigma\left(\bar{Q}^{\sim 1} \bar{Q} \mathcal{P}^{n}\right) \subset \mathcal{B} .
$$

To prove (3.3), we first observe that

$$
\lim _{t \rightarrow \infty} y_{2}^{x, 0}(t)=0 \forall x \in \mathbb{R}^{n} \Leftrightarrow \lim _{k \rightarrow \infty} y_{2}^{x, 0}(k n T)=0 \forall x \in \mathbb{R}^{n} .
$$

Indeed, we only need to show the right side of (3.5) implies the left side of (3.5), since the reverse is obvious. For this purpose, we write $\Phi_{A_{22}}(\cdot)$ for the fundamental solution associated with $A_{22}(\cdot)$. For each $t \geq 0$, let $N(t)$ be the non-negative integer such that $N(t) n T<t \leq(N(t)+1) n T$. By the $T$-periodicity and the boundedness of $A_{22}(\cdot)$, there is a positive constant $C$ such that

$$
\begin{aligned}
& \left\|y_{2}^{x, 0}(t)\right\|=\left\|\Phi_{A_{22}}(t-N(t) n T) \Phi_{A_{22}}(N(t) n T) y_{2}^{x, 0}(0)\right\| \\
\leq & C\left\|y_{2}^{x, 0}(N(t) n T)\right\| \text { for all } t \geq 0 \text { and } x \in \mathbb{R}^{n} .
\end{aligned}
$$

This yields (3.5). Then, we can easily derive from Lemma 2.4 (see (2.15)) that

$$
y_{2}^{x, 0}(k n T)=\left(\bar{Q}^{\sim 1} \bar{Q} \mathcal{P}^{n}\right)^{k} y_{2}^{x, 0}(0) \in V^{\perp} \text { for all } k \in \mathbb{N} \text { and } x \in \mathbb{R}^{n} .
$$

It is clear that $\left\{y_{2}^{x, 0}(0) \mid x \in \mathbb{R}^{n}\right\}=V^{\perp}$. This, along with (3.5) and (3.6), gives (3.3).

We next verify (3.4). It is well-known that the right side of (3.4) implies the left side of (3.4) (see Appendix C5 in $[20])$. Now we show the reverse. From Lemma 2.6 where $M=\bar{Q}$, it follows that

$$
\mathcal{P}^{n} z-\bar{Q}^{\sim 1} \bar{Q} \mathcal{P}^{n} z \in \mathcal{N} \bar{Q} \text { for each } z \in \mathbb{R}^{n} .
$$


On the other hand, by Lemmas 2.1 and 2.3 , we have

$$
z \in V \Rightarrow \mathcal{P}^{n} z \in V=\mathcal{N}(\bar{Q}) .
$$

By (3.7) and (3.8), we find that $\bar{Q}^{\sim 1} \bar{Q}^{n} z=0$ for all $z \in V$. From this and the left side of (3.4), it follows that

$$
\lim _{k \rightarrow \infty}\left(\bar{Q}^{\sim 1} \bar{Q} \mathcal{P}^{n}\right)^{k} z=0 \text { for all } z \in \mathbb{R}^{n} .
$$

This yields the right side of (3.4) (see Appendix C5 in [20]). Hence, the claim (3.2) has been proved.

Now, we suppose that $(a)$ (in Thm. 1.1) stands. Let $K(\cdot)$ be an $n T$-periodic stabilization law for the pair $[A(\cdot), B(\cdot)]$. Consider the following equation:

$$
\dot{y}(t)=[A(t)+B(t) K(t)] y(t), \quad t \in \mathbb{R}^{+} ; \quad y(0)=x .
$$

For each $x \in \mathbb{R}^{n}$, we denoted by $y(\cdot ; 0, x)$ the unique solution of equation $(3.9)$. Since $K(\cdot)$ is a feedback stabilization law, we have

$$
\lim _{t \rightarrow \infty} y(t ; 0, x)=0 \text { for each } x \in \mathbb{R}^{n} .
$$

For each $x \in \mathbb{R}^{n}$, we denote by $\left(y_{1}^{x}(\cdot), y_{2}^{x}(\cdot)\right)$ the decomposition of the solution $y(\cdot ; 0, x)$ provided by Lemma 2.4 where $u(\cdot)=K(\cdot) y(\cdot ; 0, x)$. By $(2.14)$, we have that $\left\langle y_{1}^{x}(t), y_{2}^{x}(t)\right\rangle=0$ for all $t \geq 0$, from which, it follows that

$$
\left\|y_{2}^{x}(t)\right\| \leq \sqrt{\left\|y_{1}^{x}(t)\right\|^{2}+\left\|y_{2}^{x}(t)\right\|^{2}}=\|y(t ; 0, x)\| \text { for all } t \geq 0 .
$$

This, along with (3.10), yields that

$$
\lim _{t \rightarrow \infty} y_{2}^{x}(t)=0 \text { for each } x \in \mathbb{R}^{n} .
$$

On the other hand, if we write $\bar{u}^{x}(\cdot) \triangleq K(\cdot) y(\cdot ; 0, x)$, then $y\left(\cdot ; 0, x, \bar{u}^{x}\right)=y(\cdot ; 0, x)$. Thus, $y_{2}^{x, \bar{u}^{x}}(\cdot)=y_{2}^{x}(\cdot)$. This, along with (3.1), indicates that

$$
y_{2}^{x}(t)=y_{2}^{x, 0}(t) \text {, when } t \geq 0, x \in \mathbb{R}^{n} .
$$

In summary, we conclude from (3.12), (3.11) and (3.2) that $\sigma\left(\bar{Q}^{\sim 1} \bar{Q}^{n}\right) \subset \mathcal{B}$.

Step 2 in Part 1: To show $\sigma\left(\bar{Q}^{\sim 1} \bar{Q} \mathcal{P}^{n}\right) \subset \mathcal{B} \Rightarrow(a)$

Suppose that $\sigma\left(\bar{Q}^{\sim 1} \bar{Q} \mathcal{P}^{n}\right) \subset \mathcal{B}$. By the part (i) of Lemma 2.3 and (1.7), there is an $\varepsilon_{0}>0$ such that $\left\|Q_{n}^{\varepsilon}(0)-\bar{Q}\right\|<1$, when $0<\varepsilon \leq \varepsilon_{0}$. We arbitrarily fix an $\varepsilon \in\left(0, \varepsilon_{0}\right]$, and then write

$$
\lambda_{1} \triangleq \lambda_{1}(\varepsilon) \triangleq\left\|Q_{n}^{\varepsilon}(0)-\bar{Q}\right\|^{1 / 2}=\left\|\left(S_{n}^{\varepsilon}(0)\right)^{-1}-\bar{Q}\right\|^{\frac{1}{2}}<1 .
$$

Let $K_{n}^{\varepsilon}(\cdot)$ be given by (1.8). It suffices to show that $K_{n}^{\varepsilon}(\cdot)$ is an $n T$-periodic stabilization law for $[A(\cdot), B(\cdot)]$.

For this purpose, we write $\Psi_{\varepsilon}(\cdot)$ for the fundamental solution associated with $A(\cdot)+B(\cdot) K_{n}^{\varepsilon}(\cdot)$ and write $\mathcal{P}_{\varepsilon} \triangleq \Psi_{\varepsilon}(n T)$. Let $y^{\varepsilon}\left(\cdot ; t_{0}, x\right)$ be the unique solution to the equation

$$
\dot{y}(t)=\left(A(t)+B(t) K_{n}^{\varepsilon}(t)\right) y(t), \quad t \geq 0,
$$

with the initial condition $y\left(t_{0}\right)=x$, where $t_{0} \geq 0$ and $x \in \mathbb{R}^{n}$. Clearly, $y^{\varepsilon}\left(\cdot ; t_{0}, x\right)$ is also the unique solution to equation $(1.1)$, where $u(\cdot)=K_{n}^{\varepsilon}(\cdot) y^{\varepsilon}\left(\cdot ; t_{0}, x\right)$, with the initial condition $y\left(t_{0}\right)=x$. Write $\left(y_{1}\left(\cdot ; t_{0}, x\right), y_{2}\left(\cdot ; t_{0}, x\right)\right)$ for the decomposition of $y^{\varepsilon}\left(\cdot ; t_{0}, x\right)$ provided by Lemma 2.4. (we omit $\varepsilon$ in the notation of the decomposition pair to simplify the notation). Then the pair $\left(y_{1}\left(\cdot ; t_{0}, x\right), y_{2}\left(\cdot ; t_{0}, x\right)\right)$ satisfies $(2.13)$ (where $u(\cdot)=K_{n}^{\varepsilon}(\cdot) y^{\varepsilon}\left(\cdot ; t_{0}, x\right)$ ), (2.14) and (2.15). The key is to show that

$$
\exists \bar{k} \in \mathbb{N} \text {, s.t. } \lim _{j \rightarrow \infty} y^{\varepsilon}(j \bar{k} n T ; 0, x)=0 \text { for all } x \in \mathbb{R}^{n} .
$$


When (3.15) is proved, we have $\sigma\left(\mathcal{P}_{\varepsilon}\right) \subset \mathcal{B}$ (see Appendix C5 in [20]). Hence, equation (3.14) is exponentially stable ((see [18] or [17]), i.e., $K_{n}^{\varepsilon}(\cdot)$ is an $n T$-periodic stabilization law for $[A(\cdot), B(\cdot)]$.

The rest of this step is to show (3.15). the proof is built upon the following two cases:

The first case where $x \in V$ : We first claim that

$$
\left\|y^{\varepsilon}(n T ; 0, z)\right\| \leq \lambda_{1}\|z\| \text { for all } z \in V .
$$

In fact, it follows from Lemma 2.3 (see (2.9)) that

$$
\langle z, \bar{Q} z\rangle=0 \text { for all } z \in V .
$$

On the other hand, from Lemma 2.2, the control $\bar{u}_{z}^{\varepsilon}(\cdot)$ (whit $z \in V$ ) defined by

$$
\bar{u}_{z}^{\varepsilon}(t) \triangleq K_{n}^{\varepsilon}(t) y^{\varepsilon}(t ; 0, z)=-\frac{1}{\varepsilon} B^{*}(t) Q_{n}^{\varepsilon}(t) y^{\varepsilon}(t ; 0, z) \text { for a.e. } t \in[0, n T],
$$

is the optimal control to Problem $(L Q)_{0, z}^{\varepsilon}$. This, along with (2.5), (3.13) and (3.17), indicates

$$
\left.\| y^{\varepsilon}(n T ; 0, z)\right)\left\|^{2} \leq W^{\varepsilon}(0, z)=\left\langle z, Q_{n}^{\varepsilon}(0) z\right\rangle \leq \lambda_{1}^{2}\right\| z\left\|^{2}+\langle z, \bar{Q} z\rangle=\lambda_{1}^{2}\right\| z \|^{2},
$$

which leads to (3.16). Next, since $x \in V$, it follows from (2.12) and (2.14) that $y_{2}(0 ; 0, x)=0$. Then, by the second equation in system $(2.13)$, we find that $y_{2}(\cdot ; 0, x) \equiv 0$. From $(2.2)$ and $(2.14)$, we see that

$$
y^{\varepsilon}(k n T ; 0, x)=y_{1}(k n T ; 0, x) \in \Phi(k n T) V=\mathcal{P}^{k n} V=V \text { for all } k \in \mathbb{N} .
$$

Here and throughout the proof, $\Phi(\cdot)$ denotes the fundamental solution associated with $A(\cdot)$. Let $z=$ $y^{\varepsilon}(k n T ; 0, x)$. Then by the $n T$-periodicity of $\left(A(\cdot)+B(\cdot) K_{n}^{\varepsilon}(\cdot)\right)$,

$$
y^{\varepsilon}(n T ; 0, z)=y^{\varepsilon}((k+1) n T ; 0, x) .
$$

Thus, it follows from (3.16) that

$$
\left\|y^{\varepsilon}((k+1) n T ; 0, x)\right\| \leq \lambda_{1}\left\|y^{\varepsilon}(k n T ; 0, x)\right\| \text { for all } k \in \mathbb{N} \text { and } x \in V .
$$

Since $\lambda_{1}<1$ (see $\left.(3.13)\right)$, it holds that

$$
\lim _{k \rightarrow \infty} y^{\varepsilon}(k n T ; 0, x)=0 \text { for all } x \in V .
$$

The second case when $x \in V^{\perp}$ : Since $\sigma\left(\bar{Q}^{\sim 1} \bar{Q} \mathcal{P}^{n}\right) \subset \mathcal{B}$, there is a natural number $\bar{k}$ such that (see Appendix C5 in [20])

$$
\lambda_{2} \triangleq\left\|\left(\bar{Q}^{\sim 1} \bar{Q} \mathcal{P}^{n}\right)^{\bar{k}}\right\|<1
$$

Let

$$
a_{j}=y_{1}(j \bar{k} n T ; 0, x) \text { and } \beta_{j}=y_{2}(j \bar{k} n T ; 0, x), j=0,1,2, \ldots
$$

Clearly, when $x \in V^{\perp}, \alpha_{j}+\beta_{j}=y^{\varepsilon}(j \bar{k} n T ; 0, x)$ for all $j \in \mathbb{N} ; \alpha_{0}=0$ and $\beta_{0}=x$. By the $n T$-periodicity of $\left(A(\cdot)+B(\cdot) K_{n}^{\varepsilon}(\cdot)\right)$, one can easily check that

$$
\alpha_{j+1}=y^{\varepsilon}\left(\bar{k} n T ; 0, \alpha_{j}\right)+y^{\varepsilon}\left(\bar{k} n T ; 0, \beta_{j}\right)-\beta_{j+1} .
$$

Because $\left\{\alpha_{j}\right\}_{j=0}^{\infty} \subset V$ (see (2.14) and (2.2)), it follows from (3.18) that

$$
\left\|y^{\varepsilon}\left(\bar{k} n T ; 0, \alpha_{j}\right)\right\| \leq \lambda_{1}^{\bar{k}}\left\|\alpha_{j}\right\| \text { for all } j=0,1,2, \ldots
$$


Write $\Psi_{\varepsilon}(\cdot)$ for the fundamental solution associated with $\left(A(\cdot)+B(\cdot) K_{n}^{\varepsilon}(\cdot)\right)$. Clearly,

$$
\left\|y^{\varepsilon}\left(\bar{k} n T ; 0, \beta_{j}\right)\right\| \leq\left\|\Psi_{\varepsilon}(\bar{k} n T)\right\| \cdot\left\|\beta_{j}\right\| .
$$

On the other hand, by the definition of $\beta_{j}$ and the $T$-periodicity of $A_{22}(\cdot)$ (see Lem. 2.4), we can easily check that

$$
\beta_{j+1}=y_{2}\left(\bar{k} n T ; 0, \beta_{j}\right) \text { for all } j=0,1, \ldots
$$

and

$$
y_{2}\left(k n T ; 0, \beta_{j}\right)=y_{2}\left(n T ; 0, y_{2}\left((k-1) n T ; 0, \beta_{j}\right)\right) \text { for all } k \in \mathbb{N} \text { and } j=0,1,2, \ldots
$$

By these, by using (2.15) repeatedly and then by (3.20), we can obtain that

$$
\left\|\beta_{j+1}\right\|=\left\|\left(\bar{Q}^{\sim 1} \bar{Q} \mathcal{P}^{n}\right)^{\bar{k}} \beta_{j}\right\| \leq \lambda_{2}\left\|\beta_{j}\right\| \text { for all } j=0,1,2, \ldots
$$

Now, from (3.22)-(3.25), one can deduce the estimate

$$
\left\|\alpha_{j+1}\right\| \leq \lambda_{1}^{\bar{k}}\left\|\alpha_{j}\right\|+\left(\lambda_{2}+\left\|\Psi_{\varepsilon}(\bar{k} n T)\right\|\right)\left\|\beta_{j}\right\| \text { for all } j=0,1,2, \ldots,
$$

which together with (3.25), implies that

$$
\left(\begin{array}{l}
\left\|\alpha_{j+1}\right\| \\
\left\|\beta_{j+1}\right\|
\end{array}\right) \leq\left(\begin{array}{cc}
\lambda_{1}^{\bar{k}} & \lambda_{2}+\left\|\Psi_{\varepsilon}(\bar{k} n T)\right\| \\
\lambda_{2}
\end{array}\right)\left(\begin{array}{l}
\left\|\alpha_{j}\right\| \\
\left\|\beta_{j}\right\|
\end{array}\right) \text { for all } j=0,1,2, \ldots
$$

Because $\lambda_{1}^{\bar{k}}, \lambda_{2}<1$, it holds that $\lim _{j \rightarrow \infty} \alpha_{j}=\lim _{j \rightarrow \infty} \beta_{j}=0$. Thus, we have

$$
\lim _{j \rightarrow \infty} y^{\varepsilon}(j \bar{k} n T ; 0, x)=0 \text { for all } x \in V^{\perp},
$$

since $y^{\varepsilon}(j \bar{k} n T ; 0, x)=\alpha_{j}+\beta_{j}$. Now, the key statement (3.15) follows from (3.19) and (3.27).

Step 3 in Part 1 . To verify $(c) \Leftrightarrow \sigma\left(\bar{Q}^{\sim 1} \bar{Q} \mathcal{P}^{n}\right) \subset \mathcal{B}$

Since $V$ is an invariant subspace of $\mathcal{P}$ (see $(2.2)$ ), we have $V=V_{1}(\mathcal{P}) \oplus V_{2}(\mathcal{P})$ (see Rem. 2.8). Let us recall (2.32). Because $V_{1}(\mathcal{P}) \subseteq \mathbb{R}_{1}^{n}(\mathcal{P})$ and $V_{2}(\mathcal{P}) \subseteq \mathbb{R}_{2}^{n}(\mathcal{P})$ (see Lem. 2.9), we can take $\left\{\xi_{1}, \ldots, \xi_{k_{2}}, \ldots, \xi_{k_{3}}\right\}$ as a basis of $\mathbb{R}_{1}^{n}(\mathcal{P})$, where $\Theta_{1} \triangleq\left\{\xi_{1}, \xi_{2}, \ldots, \xi_{k_{2}}\right\}$ is a basis of $V_{1}(\mathcal{P})$; and take $\left\{\eta_{1}, \ldots, \eta_{k_{1}-k_{2}}, \ldots, \eta_{n-k_{3}}\right\}$ to be a basis of $\mathbb{R}_{2}^{n}(\mathcal{P})$, where $\Theta_{3} \triangleq\left\{\eta_{1}, \eta_{2}, \ldots, \eta_{k_{1}-k_{2}}\right\}$ is a basis of $V_{2}(\mathcal{P})$. Write $\Theta_{2} \triangleq\left\{\xi_{k_{2}+1}, \xi_{k_{2}+2}, \ldots, \xi_{k_{3}}\right\}$ and $\Theta_{4} \triangleq\left\{\eta_{k_{1}-k_{2}+1}, \eta_{k_{1}-k_{2}+2}, \ldots, \eta_{n-k_{3}}\right\}$. Since $V_{1}(\mathcal{P}), V_{2}(\mathcal{P}), \mathbb{R}_{1}^{n}(\mathcal{P})$ and $\mathbb{R}_{2}^{n}(\mathcal{P})$ are invariant subspaces of $\mathcal{P}$, there are matrices $A_{1} \in \mathbb{R}^{k_{2} \times k_{2}}, A_{12} \in \mathbb{R}^{k_{2} \times\left(k_{3}-k_{2}\right)}, A_{2} \in \mathbb{R}^{\left(k_{3}-k_{2}\right) \times\left(k_{3}-k_{2}\right)}, A_{3} \in \mathbb{R}^{\left(k_{1}-k_{2}\right) \times\left(k_{1}-k_{2}\right)}$, $A_{34} \in \mathbb{R}^{\left(k_{1}-k_{2}\right) \times\left(n-k_{3}-k_{1}+k_{2}\right)}$ and $A_{4} \in \mathbb{R}^{\left(n-k_{3}-k_{1}+k_{2}\right) \times\left(n-k_{3}-k_{1}+k_{2}\right)}$ such that

$$
\mathcal{P}\left(\Theta_{1}, \Theta_{2}, \Theta_{3}, \Theta_{4}\right)=\left(\Theta_{1}, \Theta_{2}, \Theta_{3}, \Theta_{4}\right)\left(\begin{array}{cccc}
A_{1} & A_{12} & 0 & 0 \\
0 & A_{2} & 0 & 0 \\
0 & 0 & A_{3} & A_{34} \\
0 & 0 & 0 & A_{4}
\end{array}\right) .
$$

Then, there are matrices $\hat{A}_{12} \in \mathbb{R}^{k_{2} \times\left(k_{3}-k_{2}\right)}$ and $\hat{A}_{34} \in \mathbb{R}^{\left(k_{1}-k_{2}\right) \times\left(n-k_{3}-k_{1}+k_{2}\right)}$ such that

$$
\mathcal{P}^{n}\left(\Theta_{1}, \Theta_{2}, \Theta_{3}, \Theta_{4}\right)=\left(\Theta_{1}, \Theta_{2}, \Theta_{3}, \Theta_{4}\right)\left(\begin{array}{cccc}
A_{1}^{n} & \hat{A}_{12} & 0 & 0 \\
0 & A_{2}^{n} & 0 & 0 \\
0 & 0 & A_{3}^{n} & \hat{A}_{34} \\
0 & 0 & 0 & A_{4}^{n}
\end{array}\right) .
$$


On the other hand, by $(2.27)$ and $(2.9), I-\bar{Q}^{\sim 1} \bar{Q}$ is a linear transform from $\mathbb{R}^{n}$ to $V=V_{1}(\mathcal{P}) \bigoplus V_{2}(\mathcal{P})$. Thus, there are vectors $c_{1}, \ldots, c_{k_{1}}$ in $\mathbb{R}^{n}$ such that

$$
\left(I-\bar{Q}^{\sim 1} \bar{Q}\right) \zeta=\sum_{i=1}^{k_{2}}\left\langle\zeta, c_{i}\right\rangle \xi_{i}+\sum_{i=1}^{k_{1}-k_{2}}\left\langle\zeta, c_{k_{2}+i}\right\rangle \eta_{i} \text { for all } \zeta \in \mathbb{R}^{n},
$$

which leads to

$$
\bar{Q}^{\sim 1} \bar{Q} \zeta=\zeta-\sum_{i=1}^{k_{2}}\left\langle\zeta, c_{i}\right\rangle \xi_{i}-\sum_{i=1}^{k_{1}-k_{2}}\left\langle\zeta, c_{k_{2}+i}\right\rangle \eta_{i} \text { for all } \zeta \in \mathbb{R}^{n} .
$$

This, together with the fact that $\bar{Q} V=\{0\}$ (see (2.9)), yields that there are matrices $C_{1} \in \mathbb{R}^{k_{2} \times\left(k_{3}-k_{2}\right)}$, $C_{2} \in \mathbb{R}^{\left(k_{1}-k_{2}\right) \times\left(k_{3}-k_{2}\right)}, C_{3} \in \mathbb{R}^{k_{2} \times\left(n-k_{3}-k_{1}+k_{2}\right)}, C_{4} \in \mathbb{R}^{\left(k_{1}-k_{2}\right) \times\left(n-k_{3}-k_{1}+k_{2}\right)}$ such that

$$
\bar{Q}^{\sim 1} \bar{Q}\left(\Theta_{1}, \Theta_{2}, \Theta_{3}, \Theta_{4}\right)=\left(\Theta_{1}, \Theta_{2}, \Theta_{3}, \Theta_{4}\right)\left(\begin{array}{cccc}
0 & C_{1} & 0 & C_{3} \\
0 & I_{k_{3}-k_{2}} & 0 & 0 \\
0 & C_{2} & 0 & C_{4} \\
0 & 0 & 0 & I_{n-k_{3}-k_{1}+k_{2}}
\end{array}\right) .
$$

From (3.28) and (3.30), it follows that

$$
\bar{Q}^{\sim 1} \bar{Q} \mathcal{P}\left(\Theta_{1}, \Theta_{2}, \Theta_{3}, \Theta_{4}\right)=\left(\Theta_{1}, \Theta_{2}, \Theta_{3}, \Theta_{4}\right)\left(\begin{array}{cccc}
0 & C_{1} A_{2} & 0 & C_{3} A_{4} \\
0 & A_{2} & 0 & 0 \\
0 & C_{2} A_{2} & 0 & C_{4} A_{4} \\
0 & 0 & 0 & A_{4}
\end{array}\right),
$$

which yields that

$$
\sigma\left(\bar{Q}^{\sim 1} \bar{Q} \mathcal{P}\right)= \begin{cases}\sigma\left(A_{2}\right) \bigcup \sigma\left(A_{4}\right) \bigcup\{0\}, & \text { if } k_{1} \geq 1 \\ \sigma\left(A_{2}\right) \bigcup \sigma\left(A_{4}\right), & \text { if } k_{1}=0 .\end{cases}
$$

Similarly, it follows from (3.29) and (3.30) that

$$
\sigma\left(\bar{Q}^{\sim 1} \bar{Q}^{n}\right)= \begin{cases}\sigma\left(A_{2}^{n}\right) \bigcup \sigma\left(A_{4}^{n}\right) \bigcup\{0\}, & \text { if } k_{1} \geq 1 \\ \sigma\left(A_{2}^{n}\right) \bigcup \sigma\left(A_{4}^{n}\right), & \text { if } k_{1}=0 .\end{cases}
$$

By (3.31) and (3.32), we see that

$$
\sigma\left(\bar{Q}^{\sim 1} \bar{Q} \mathcal{P}^{n}\right)=\left\{\lambda^{n} \mid \lambda \in \sigma\left(\bar{Q}^{\sim 1} \bar{Q} \mathcal{P}\right)\right\} .
$$

Therefore, it holds that $\sigma\left(\bar{Q}^{\sim 1} \bar{Q} \mathcal{P}\right) \subset \mathcal{B} \Leftrightarrow \sigma\left(\bar{Q}^{\sim 1} \bar{Q} \mathcal{P}^{n}\right) \subset \mathcal{B}$, i.e., $(c) \Leftrightarrow \sigma\left(\bar{Q}^{\sim 1} \bar{Q} \mathcal{P}^{n}\right) \subset \mathcal{B}$.

In summary, we conclude that the proof of Part 1 is finished.

Part 2. To show $(a) \Leftrightarrow(b)$

Clearly, $(a) \Leftrightarrow(b)$ in the case that $n=1$. Thus, we can assume that $n \geq 2$. It is obvious that $(b) \Rightarrow(a)$. Now we show that $(a) \Leftrightarrow(b)$ for the case that $n \geq 2$. Suppose that $(a)$ stands and $n \geq 2$. It suffices to show that there is a $T$-periodic stabilization law for $[A(\cdot), B(\cdot)]$. To verify this, we first construct a special $n \times n$ real matrix $X$ (which will appear in (1.6)), then provide an $\varepsilon_{0}>0$ (depending on $n,\|X\|$ and $\|\mathcal{P}\|$ ), and finally prove that when $\varepsilon \in\left(0, \varepsilon_{0}\right], K^{\varepsilon}(\cdot)$, given by (1.9) with the aforementioned $X$, is a $T$-periodic feedback stabilization law for $[A(\cdot), B(\cdot)]$. The detailed proof will be carried by several steps. 
Step 1 in Part 2. Structure of $X$ in (1.6) where $n \geq 2$

Recall that $k_{1}=\operatorname{dim} V$ (see $\left.(2.32)\right)$. Hence, $\operatorname{dim} V^{\perp}=n-k_{1}$. We arbitrarily take a basis $\left\{\hat{\eta}_{1}, \ldots, \hat{\eta}_{n-k_{1}}\right\}$ of $V^{\perp}$. The desired $X$ will be defined by

$$
X \triangleq\left(\zeta_{1}, \ldots, \zeta_{k_{1}}, \hat{\eta}_{1}, \ldots, \hat{\eta}_{n-k_{1}}\right)
$$

Here, $\left\{\zeta_{1}, \ldots, \zeta_{k_{1}}\right\}$ is a special basis of $V$, which will be determined later. Clearly, $X$ is invertible. To construct the aforementioned basis $\left\{\zeta_{1}, \ldots, \zeta_{k_{1}}\right\}$, we will build up subspaces $W_{1}, W_{2}, \ldots$ and $W_{n}$ of $V$ such that

$$
V_{j}=\bigoplus_{i=1}^{j} W_{i} \text { for all } j \in\{1,2, \ldots, n\}
$$

and

$$
\mathcal{P} W_{j+1} \subseteq W_{j} \text { for all } j \in\{1, \ldots, n-1\} .
$$

Here, we agree that $\{0\}$ is the 0 -dimension subspace of $\mathbb{R}^{n}$.

When the above-mentioned $\left\{W_{1}, \ldots, W_{n}\right\}$ is structured, it follows accordingly from (3.35) and (3.36) that

$$
V=V_{n}=\bigoplus_{i=1}^{n} W_{i}
$$

and

The latter implies that

$$
\operatorname{dim} W_{j+1} \leq \operatorname{dim} W_{j} \text { for each } j \in\{1, \ldots, n-1\} .
$$

$$
W_{j+1}=\{0\}, \text { whenever } W_{j}=\{0\} \text { for some } j \in\{1, \ldots, n-1\} .
$$

Write $\left\{\hat{\zeta}_{1}, \ldots, \hat{\zeta}_{\hat{k}_{1}}\right\},\left\{\hat{\zeta}_{\hat{k}_{1}+1}, \ldots, \hat{\zeta}_{\hat{k}_{2}}\right\}, \ldots$, and $\left\{\hat{\zeta}_{\hat{k}_{n-1}+1}, \ldots, \hat{\zeta}_{\hat{k}_{n}}\right\}$ for bases of $W_{1}, W_{2}, \ldots$, and $W_{n}$, respectively. Here, we agree that any basis of $W_{j}$ is $\varnothing$, if $W_{j}=\{0\}$. From the fact that $k_{1}=\operatorname{dim} V,(3.37)$ and (3.38), it follows that $\left\{\hat{\zeta}_{1}, \ldots, \hat{\zeta}_{k_{1}}\right\}$ is a basis of $V$. Then we take the desired basis $\left\{\zeta_{1}, \ldots, \zeta_{k_{1}}\right\}$ in $(3.34)$ to be $\left\{\hat{\zeta}_{1}, \ldots, \hat{\zeta}_{k_{1}}\right\}$.

The rest of this step is to structure $\left\{W_{1}, \ldots, W_{n}\right\}$ satisying (3.35) and (3.36). Two observations are given in order:

$$
V_{j}=\mathcal{P}^{-1} V_{j-1}+V_{j-1} \text { for all } j \in\{2, \ldots, n\}
$$

and

$$
V_{j}=V_{j-1}+\mathcal{P}^{-(j-1)} V_{1} \text { for all } j \in\{2, \ldots, n\} .
$$

Now we construct the above-mentioned $\left\{W_{1}, \ldots, W_{n}\right\}$ by the following two cases:

The first case when $n=2$. We first take $W_{1}=V_{1}$. Then, from (3.40) where $j=2$, we see that $V_{2}=V_{1}+\mathcal{P}^{-1} V_{1}$, by which, there is a subspace $W_{2}$ such that $V_{2}=V_{1} \oplus W_{2}$ and $W_{2} \subset \mathcal{P}^{-1} V_{1}$. Hence, $\left\{W_{1}, W_{2}\right\}$ satisfies (3.35) and (3.36) in the case that $n=2$.

The second case that $n>2$. Let $W_{1}=V_{1}$. Now we build up $\left\{W_{2}, \ldots, W_{n}\right\}$ in such an order: $W_{n} \rightarrow W_{n-1} \rightarrow$ $\ldots \rightarrow W_{2}$. By (3.40) where $j=n$, there is a subspace $W_{n}$ such that

$$
V_{n}=V_{n-1} \bigoplus W_{n} \text { and } W_{n} \subseteq \mathcal{P}^{-(n-1)} V_{1} .
$$

Then, from the second property in (3.41), it follows that

$$
\mathcal{P} W_{n} \subseteq \mathcal{P}^{-(n-2)} V_{1} .
$$

Besides, it holds that

$$
\mathcal{P} W_{n} \bigcap V_{n-2}=\{0\} .
$$


In fact, if $y \in \mathcal{P} W_{n} \bigcap V_{n-2}$, then $y=\mathcal{P} z$ for some $z \in W_{n}$. Thus, $z=\mathcal{P}^{-1} y \in \mathcal{P}^{-1} V_{n-2}$. This, along with (3.39) where $j=n-1$, indicates that $z \in V_{n-1}$. From this and the facts that $z \in W_{n}$ and $V_{n-1} \cap W_{n}=\{0\}$ (see the first property in (3.41)), it follows that $z=0$. Hence, $y=\mathcal{P} z=0$, which leads to (3.43).

We next build up $W_{n-1}$. From (3.40) where $j=n-1,(3.42)$ and (3.43), we see that

$$
V_{n-1}=V_{n-2}+\mathcal{P}^{-(n-2)} V_{1}=V_{n-2}+\mathcal{P}^{-(n-2)} V_{1}+\mathcal{P} W_{n}=\left(V_{n-2} \bigoplus \mathcal{P} W_{n}\right)+\mathcal{P}^{-(n-2)} V_{1} .
$$

Thus, there is subspace $\hat{W}_{n-1}$ such that

$$
\hat{W}_{n-1} \subseteq \mathcal{P}^{-(n-2)} V_{1} \text { and } V_{n-1}=\left(V_{n-2} \bigoplus \mathcal{P} W_{n}\right) \bigoplus \hat{W}_{n-1} .
$$

Let

$$
W_{n-1}=\mathcal{P} W_{n} \bigoplus \hat{W}_{n-1} .
$$

It is clear that

$$
V_{n-1}=V_{n-2} \bigoplus W_{n-1} \text { and } \mathcal{P} W_{n} \subseteq W_{n-1} .
$$

Besides, from (3.45), (3.42) and the first result in (3.44), we obtain that

$$
\mathcal{P} W_{n-1} \subseteq \mathcal{P}^{-(n-3)} V_{1} .
$$

By (3.39), with $j=n-2$, and the fact that $V_{n-2} \bigcap W_{n-1}=\{0\}$ (see the first fact in (3.46)), using the same method to show (3.43), we can easily verify that

$$
\mathcal{P} W_{n-1} \bigcap V_{n-3}=\{0\} .
$$

By (3.47) and (3.48), following the same way to construct $W_{n-1}$, we can build up a subspace $W_{n-2}$ with the similar properties as those in (3.46), (3.47) and (3.48). Then we can structure, step by step, subspaces $W_{n-3}, \ldots, W_{2}$ such that

$$
V_{j}=V_{j-1} \bigoplus W_{j} \text { and } \mathcal{P} W_{j+1} \subseteq W_{j} \text { for all } j \in\{n-2, \ldots, 2\} .
$$

Now, from the first property in (3.41), (3.46), (3.49) and the fact that $W_{1}=V_{1}$, one can easily check that the subspaces $W_{1}, \ldots, W_{n}$ built up above satisfy (3.35) and (3.36) in the case that $n>2$.

We end this step with the following property which will be used later:

$$
\left\langle X^{-1} z_{1}, X^{-1} z_{2}\right\rangle=0 \text { for all } z_{1} \in W_{i}, z_{2} \in W_{j} \text {, with } i \neq j \text { and } i, j \in\{1,2, \ldots, n\} .
$$

The property (3.50) can be easily verified, since $W_{j} \cap W_{i}=\{0\}$ for all $i, j=1, \ldots, n$ with $i \neq j$.

Step 2 in Part 2. Structure of a T-periodic $K^{\varepsilon}(\cdot)$ in $L^{\infty}\left(\mathbb{R}^{+} ; \mathbb{R}^{m \times n}\right)$ and a positive number $\varepsilon_{0}$

Let $X$ be given by (3.34). For each $\varepsilon>0$ and $x \in \mathbb{R}^{n}$, consider the optimal control problem

$$
\left(P_{x}^{\varepsilon}\right): \quad \hat{W}^{\varepsilon}(t, x) \triangleq \inf _{u \in L^{2}\left(0, T ; \mathbb{R}^{m}\right)} \hat{J}^{\varepsilon}(u ; x) .
$$

Here $\hat{J}^{\varepsilon}(\cdot ; x): L^{2}\left(0, T ; \mathbb{R}^{m}\right) \rightarrow \mathbb{R}^{+}$is defined by

$$
\hat{J}^{\varepsilon}(u ; x) \triangleq \int_{0}^{T} \varepsilon\langle u(t), u(t)\rangle \mathrm{d} t+\left\langle X^{-1} \mathcal{P}^{-1} y(T ; 0, x, u), \quad X^{-1} \mathcal{P}^{-1} y(T ; 0, x, u)\right\rangle,
$$


where $y(\cdot ; 0, x, u)$ is the solution of equation $(1.1)$ over $[0, T]$, with the initial condition $y(0)=x$. According to Theorem 37 on page 364 in [20] (see also Lem. 2.2),

$$
\hat{W}^{\varepsilon}(t, x)=\left\langle x, \hat{Q}^{\varepsilon}(t) x\right\rangle \text { for all }(t, x) \in[0, T) \times \mathbb{R}^{n},
$$

where $\hat{Q}^{\varepsilon}(\cdot)$ is a symmetric and positive definite $n \times n$ matrix-valued function over $[0, T]$, and $S^{\varepsilon}(\cdot)=\left(\hat{Q}^{\varepsilon}(\cdot)\right)^{-1}$ is the unique solution to the equation (1.6).

For each $x \in V_{1}$, there is a control $u_{1} \in \mathcal{U}_{\text {ad }}$ such that $y\left(T ; 0, x, u_{1}\right)=0$. Thus, by (3.52),

$$
0 \leq \lim _{\varepsilon \rightarrow 0^{+}}\left\langle x, \hat{Q}^{\varepsilon} x>=\lim _{\varepsilon \rightarrow 0^{+}} \hat{W}^{\varepsilon}(0, x) \leq \lim _{\varepsilon \rightarrow 0^{+}} \hat{J}^{\varepsilon}\left(u_{1} ; x\right)=0 \text {, when } x \in V_{1} .\right.
$$

From this, $\lim _{\varepsilon \rightarrow 0^{+}}<x, \hat{Q}^{\varepsilon}(0) x>=0$ for each $x \in V_{1}$. Since $V_{1}$ is a finite dimensional space,

$$
\left\langle x, \hat{Q}^{\varepsilon}(0) x\right\rangle \rightarrow 0 \text { uniformly in }\left\{x \in V_{1}:\|x\| \leq 1\right\} .
$$

Let

$$
\delta \triangleq \frac{1}{2 n\|X\|\left(1+\left(\sqrt{2}\|\mathcal{P}\|\|X\|\left\|X^{-1}\right\|\right)^{n}\right)}
$$

By (3.53), there is an

$$
\varepsilon_{0} \triangleq \varepsilon_{0}(\delta) \triangleq \varepsilon_{0}(n, X, \mathcal{P})>0
$$

such that when $\varepsilon \in\left(0, \varepsilon_{0}\right]$,

$$
\left\langle x, \hat{Q}^{\varepsilon}(0) x\right\rangle \leq \delta^{2}\|x\|^{2} \text { for all } x \in V_{1} .
$$

In the rest of the proof, we fix an $\varepsilon \in\left(0, \varepsilon_{0}\right]$. Let $K^{\varepsilon}(\cdot)$ be defined by (1.9), where $X$ is given by (3.34). Write $\bar{y}^{x}(\cdot)$ for the solution to the equation

$$
\left\{\begin{array}{l}
\dot{y}(t)=\left(A(t)+B(t) K^{\varepsilon}(t)\right) y(t), \text { a.e. } t \in[0,+\infty), \\
y(0)=x .
\end{array}\right.
$$

By Theorem 37, page 364, [20] (see also Lem. 2.2), the control

$$
\bar{u}^{x}(\cdot) \triangleq K^{\varepsilon}(\cdot) \bar{y}^{x}(\cdot),
$$

when it is restricted over $(0, T)$, is the optimal control to problem $\left(P_{x}^{\varepsilon}\right)$.

Step 3 in Part 2. To prove that the above $K^{\varepsilon}(\cdot)$ is a $T$-periodic feedback stabilization law

Define a linear mapping $\mathcal{L}$ on $\mathbb{R}^{n}$ by

$$
\mathcal{L}(x)=x+\int_{0}^{T} \Phi^{-1}(s) B(s) \bar{u}^{x}(s) d s \quad \text { for all } x \in \mathbb{R}^{n},
$$

where $\bar{u}^{x}(\cdot)$ is given by $(3.58)$. Clearly,

$$
\bar{y}^{x}(T)=\mathcal{P} \mathcal{L}(x) \text { for all } x \in \mathbb{R}^{n} .
$$

First, we claim

$$
\mathcal{L}(x)=x, \quad \text { if } x \in \bigoplus_{j=2}^{n} W_{j} ; \quad \mathcal{L}(x) \in W_{1} \text { and }\|\mathcal{L}(x)\| \leq\|x\|, \quad \text { if } x \in W_{1} .
$$


To prove the first statement in (3.61), it suffices to show that $\bar{u}^{x}(\cdot) \equiv 0$ for all $x \in \bigoplus_{j=2}^{n} W_{j}$ (see (3.59)). For this purpose, we observe that for each $u \in L^{2}\left(0, T ; \mathbb{R}^{m}\right)$,

$$
\left.z \triangleq \int_{0}^{T} \Phi^{-1}(s) B(s) u(s) d s \in V_{1}=W_{1} \quad \text { (see }(2.3),(3.35), \text { where } j=1\right),
$$

and

$$
y(T ; 0, x, u)=\mathcal{P}(x+z) \text { for all } x \in \mathbb{R}^{n} .
$$

These, together with (3.50), yields that when $x \in \bigoplus_{j=2}^{n} W_{j}$,

$$
\begin{aligned}
\hat{J}^{\varepsilon}(u ; x) & =\int_{0}^{T} \varepsilon\langle u(t), u(t)\rangle \mathrm{d} t+\left\langle X^{-1} \mathcal{P}^{-1} y(T ; 0, x, u), X^{-1} \mathcal{P}^{-1} y(T ; 0, x, u)\right\rangle \\
& \geq\left\langle X^{-1} \mathcal{P}^{-1} y(T ; 0, x, u), X^{-1} \mathcal{P}^{-1} y(T ; 0, x, u)\right\rangle \\
& =\left\langle X^{-1}(x+z), X^{-1}(x+z)\right\rangle \geq\left\langle X^{-1} x, X^{-1} x\right\rangle=\hat{J}^{\varepsilon}(0 ; x) .
\end{aligned}
$$

Hence, the null control is the optimal control to problem $\left(P_{x}^{\varepsilon}\right)$. Since the optimal control to this problem is unique (see Lem. 2.2), it stands that $\bar{u}^{x}(\cdot) \equiv 0$.

To prove the second statement in (3.61), we first observe from (2.3) that $\mathcal{L} W_{1} \subseteq W_{1}$ (since $W_{1}=V_{1}$ ). Then, by the optimality of $\bar{u}^{x}$, we see that

$$
\hat{J}^{\varepsilon}\left(\bar{u}^{x} ; x\right)=<x, \hat{Q}^{\varepsilon}(0) x>\text { for all } x \in \mathbb{R}^{n} .
$$

This, together with the definition of $\hat{J}^{\varepsilon}$ and (3.56), indicates that

$$
\left\|X^{-1} \mathcal{P}^{-1} \bar{y}^{x}(T)\right\| \leq \sqrt{\hat{J}^{\varepsilon}\left(\bar{u}^{x}, x\right)}=\sqrt{\left\langle x, \hat{Q}^{\varepsilon}(0) x\right\rangle} \leq \delta\|x\| \text { for each } x \in W_{1}=V_{1} .
$$

From this and (3.60),

$$
\|\mathcal{L}(x)\|=\left\|\mathcal{P}^{-1} \bar{y}^{x}(T)\right\| \leq\|X\|\left\|X^{-1} \mathcal{P}^{-1} \bar{y}^{x}(T)\right\| \leq \delta\|X\|\|x\| \text { for each } x \in W_{1},
$$

which, along with (3.54), leads to the second statement in (3.61).

Now we conclude that the claim (3.61) stands. In addition, by (3.62), we see that

$$
\left\|\bar{y}^{x}(T)\right\|=\|\mathcal{P} \mathcal{L}(x)\| \leq\|\mathcal{P}\|\|\mathcal{L}(x)\| \leq \delta\|\mathcal{P}\|\|X\|\|x\| \text { for each } x \in W_{1} .
$$

Second, we claim

$$
\left\|\bar{y}^{x}(T)\right\| \leq \sqrt{2}\|\mathcal{P}\|\|X\|\left\|X^{-1}\right\|\|x\| \text { for each } x \in V .
$$

In fact, by (3.37), each $x \in V$ can be expressed as: $x=x_{1}+x_{2}$, where $x_{1} \in W_{1}$ and $x_{2} \in \bigoplus_{j=2}^{n} W_{j}$. Because of (3.50), vectors $X^{-1} x_{1}$ and $X^{-1} x_{2}$ are orthogonal. Thus, it holds that

$$
\left\|X^{-1} x_{1}+X^{-1} x_{2}\right\|^{2}=\left\|X^{-1} x_{1}\right\|^{2}+\left\|X^{-1} x_{2}\right\|^{2} \geq 1 / 2\left(\left\|X^{-1} x_{1}\right\|+\left\|X^{-1} x_{2}\right\|\right)^{2} .
$$

This, along with the second statement in (3.61), yields

$$
\left\|\bar{y}^{x}(T)\right\|=\left\|\bar{y}^{x_{1}}(T)+\bar{y}^{x_{2}}(T)\right\|=\left\|\mathcal{P}\left(\mathcal{L}\left(x_{1}\right)+\mathcal{L}\left(x_{2}\right)\right)\right\| \leq \sqrt{2}\|\mathcal{P}\|\|X\|\left(\left\|X^{-1} x_{1}+X^{-1} x_{2}\right\|\right),
$$

which leads to (3.64).

Next, we claim

$$
\left\|y^{x}(n T)\right\| \leq \delta\|\mathcal{P}\|^{n}\left(\sqrt{2}\|X\|\left\|X^{-1}\right\|\right)^{n-1}\|X\|\|x\| \text { for all } x \in \bigcup_{i=1}^{n} W_{i}
$$


For this purpose, we first observe from the $T$-periodicity of $A(\cdot)+B(\cdot) K^{\varepsilon}(\cdot)$ that

$$
\bar{y}^{x}(k T)=\bar{y}^{y^{x}((k-1) T)}(T) \text { for all } x \in \mathbb{R}^{n} \text { and } k \in \mathbb{N} .
$$

By (3.66) and (3.64), one can easily check that

$$
\left\|\bar{y}^{x}(n T)\right\| \leq\left(\sqrt{2}\|\mathcal{P}\|\|X\|\left\|X^{-1}\right\|\right)^{n-1}\left\|\bar{y}^{x}(T)\right\| \text { for all } x \in V
$$

This, along with (3.63), indicates that the estimate in (3.65) holds when $x \in W_{1}$, i.e.,

$$
\left\|\bar{y}^{x}(n T)\right\| \leq \delta\|\mathcal{P}\|^{n}\left(\sqrt{2}\|X\|\left\|X^{-1}\right\|\right)^{n-1}\|X\|\|x\| \text { for all } x \in W_{1} .
$$

Now, we arbitrarily fix an $x$ in $W_{j}$ for some $j \in\{2,3, \ldots, n\}$. By (3.66), (3.60), (3.61) and (3.36), using the mathematical induction, one can easily prove that

$$
\bar{y}^{x}((j-1) T)=\mathcal{P} \bar{y}^{x}((j-2) T)=\mathcal{P}^{j-1} x \in W_{1} .
$$

This, together with (3.66) and (3.63), yields that

$$
\left\|\bar{y}^{x}(j T)\right\| \leq \delta\|\mathcal{P}\|\|X\|\left\|\bar{y}^{x}((j-1) T)\right\| \leq \delta\|\mathcal{P}\|^{j}\|X\|\|x\| \text { for all } x \in W_{j} .
$$

On the other hand, by (3.64), one can easily check that

$$
\left\|\bar{y}^{z}(k T)\right\| \leq\left(\sqrt{2}\|\mathcal{P}\|\|X\|\left\|X^{-1}\right\|\right)^{k}\|z\| \text { for all } z \in V \text { and } k \in \mathbb{N} .
$$

This, along with the fact that $\bar{y}^{x}(n T)=\bar{y}^{\bar{y}^{x}(j T)}((n-j) T)$, yields that

$$
\left\|\bar{y}^{x}(n T)\right\| \leq\left(\sqrt{2}\|\mathcal{P}\|\|X\|\left\|X^{-1}\right\|\right)^{n-j}\left\|\bar{y}^{x}(j T)\right\|,
$$

which, together with (3.68), shows that

$$
\left\|\bar{y}^{x}(n T)\right\| \leq \delta\|\mathcal{P}\|^{n}\left(\sqrt{2}\|X\|\left\|X^{-1}\right\|\right)^{n-j}\|X\|\|x\|<\delta\|\mathcal{P}\|^{n}\left(\sqrt{2}\|X\|\left\|X^{-1}\right\|\right)^{n-1}\|X\|\|x\| .
$$

Since $x$ was arbitrarily taken from one of $W_{j}$ with $j \in\{2,3, \ldots, n\}$, the above estimate holds for all $x \in \bigcup_{i=2}^{n} W_{i}$. This, along with (3.67), leads to (3.65).

Then, we claim

$$
\left\|\bar{y}^{x}(n T)\right\| \leq \delta\|x\| \text { for all } x \in V .
$$

In fact, by (3.37), each $x \in V$ can be expressed as: $x=\sum_{j=1}^{n} x_{j}$ with $x_{j} \in W_{j}$ for all $j=1, \ldots, n$. Thus, it holds that

$$
\left\|\bar{y}^{x}(n T)\right\|=\left\|\bar{y}^{\sum_{1}^{n} x_{j}}(n T)\right\|=\left\|\sum_{j=1}^{n} \bar{y}^{x_{j}}(n T)\right\| \leq \sum_{j=1}^{n}\left\|\bar{y}^{x_{j}}(n T)\right\| .
$$

This, together with (3.65), yields that

$$
\left\|\bar{y}^{x}(n T)\right\| \leq \delta\|\mathcal{P}\|^{n}\left(\sqrt{2}\|X\|\left\|X^{-1}\right\|\right)^{n-1}\|X\| \sum_{j=1}^{n}\left\|x_{j}\right\| .
$$


Meanwhile, since $\left\langle X^{-1} x_{i}, X^{-1} x_{j}\right\rangle=0$ when $i \neq j$ (see (3.50)), one can directly check that

$$
n\left\|\sum_{j=1}^{n} X^{-1} x_{j}\right\|^{2}=n \sum_{j=1}^{n}\left\|X^{-1} x_{j}\right\|^{2} \geq\left(\sum_{j=1}^{n}\left\|X^{-1} x_{j}\right\|\right)^{2} .
$$

From the above two inequalities, we can easily verify that

$$
\left\|\bar{y}^{x}(n T)\right\|<\delta n\|X\|\left(\sqrt{2}\|\mathcal{P}\|\|X\|\left\|X^{-1}\right\|\right)^{n}\|x\| .
$$

This, together with (3.54), leads to (3.69).

Now, we write $\hat{\mathcal{P}}_{\varepsilon}$ for the transformation over time $T$, associated with $A(\cdot)+B(\cdot) K^{\varepsilon}(\cdot)$ (see (1.4)). It is clear that

$$
\bar{y}^{x}(n T)=\left(\hat{\mathcal{P}}_{\varepsilon}\right)^{n} x \text { for each } x \in \mathbb{R}^{n},
$$

and

$$
\bar{y}^{x}(T)=\mathcal{P}\left[x+\int_{0}^{T} \Phi^{-1}(s) B(s) \bar{u}^{x}(s) \mathrm{d} s\right] \text { for each } x \in \mathbb{R}^{n} .
$$

Here, we recall that $\Phi(\cdot)$ is the fundamental solution for $A(\cdot)$ and $\bar{u}_{\varepsilon}^{x}(\cdot)$ is given by (3.58). Then, by (2.3), we see that

$$
x+\int_{0}^{T} \Phi^{-1}(s) B(s) \bar{u}^{x}(s) \mathrm{d} s \in V, \quad \text { when } x \in V .
$$

This, along with (3.71) and (2.2), yields that $\bar{y}^{x}(T) \in V$, when $x \in V$. Thus, by making use of (2.2) again, we see that $\bar{y}^{x}(2 T)=\bar{y}^{x}(T)(T) \in V$. Then, step by step, we can reach that

$$
\bar{y}^{x}(n T)=\bar{y}^{\bar{y}^{x}((n-1) T)}(T) \in V, \quad \text { when } x \in V .
$$

This leads to

$$
\left(\hat{\mathcal{P}}_{\varepsilon}\right)^{n}: V \rightarrow V
$$

Hence, there are $\tilde{A}_{11} \in \mathbb{R}^{k_{1} \times k_{1}}, \tilde{A}_{21} \in \mathbb{R}^{k_{1} \times\left(n-k_{1}\right)}, \tilde{A}_{22} \in \mathbb{R}^{\left(n-k_{1}\right) \times\left(n-k_{1}\right)}$ such that

$$
\left(\hat{\mathcal{P}}_{\varepsilon}\right)^{n} X=X\left(\begin{array}{cc}
\tilde{A}_{11} & \tilde{A}_{12} \\
0 & \tilde{A}_{22}
\end{array}\right)
$$

By (3.73), it holds that $\sigma\left(\left.\left(\hat{\mathcal{P}}_{\varepsilon}\right)^{n}\right|_{V}\right)=\sigma\left(\tilde{A}_{11}\right)$. Then, from (3.70) and (3.69), we see that if $\delta<1$ is given by (3.54), then $\left\|\left(\hat{\mathcal{P}}_{\varepsilon}\right)^{n} x\right\| \leq \delta\|x\|$ for all $x \in V$. Since $V$ is invariant under $\left(\hat{\mathcal{P}}_{\varepsilon}\right)^{n}$ (see $(3.72)$ ), the above inequality implies that $\left\|\left.\left(\hat{\mathcal{P}}_{\varepsilon}\right)^{n}\right|_{V}\right\|<1$. Furthermore, it follows that $\sigma\left(\left.\left(\hat{\mathcal{P}}_{\varepsilon}\right)^{n}\right|_{V}\right) \subset \mathcal{B}$ (see Appendix C5 in [20]). Hence, $\sigma\left(\tilde{A}_{11}\right) \subset \mathcal{B}$. We next prove that $\sigma\left(\tilde{A}_{22}\right) \subset \mathcal{B}$. When this is done, it follows from $(3.73)$ that $\sigma\left(\left(\hat{\mathcal{P}}_{\varepsilon}\right)^{n}\right) \subset \mathcal{B}$, which leads to the facts that $\sigma\left(\hat{\mathcal{P}}_{\varepsilon}\right) \subset \mathcal{B}$ and $K^{\varepsilon}$ is a $T$-periodic feedback stabilization law for the pair $[A(\cdot), B(\cdot)]$. Thus, we complete the proof of the statement that $(a) \Leftrightarrow(b)$.

The rest of this step is to show $\sigma\left(\tilde{A}_{22}\right) \subset \mathcal{B}$. Recall that $k_{1}$ and $X=\left(\zeta_{1}, \ldots, \zeta_{k_{1}}, \hat{\eta}_{1}, \ldots, \hat{\eta}_{n-k_{1}}\right)$ are given by $(2.32)$ and (3.34), respectively. Since $\left\{\hat{\eta}_{1}, \ldots, \hat{\eta}_{n-k_{1}}\right\}$ is a basis of $V^{\perp}$ (see $\left.(3.34)\right)$, the matrix $\left(\hat{\eta}_{1}, \ldots, \hat{\eta}_{n-k_{1}}\right)$ can be treated as a linear and one-to-one map from $\mathbb{R}^{n-k_{1}}$ to $V^{\perp}$. Write

$$
z(c) \triangleq\left(\hat{\eta}_{1}, \ldots, \hat{\eta}_{n-k_{1}}\right) c \in V^{\perp} \text { for each } c \in \mathbb{R}^{n-k_{1}} .
$$

By (3.74) and (3.34), we see that

$$
z(c)=X\left(\begin{array}{c}
0_{k_{1} \times k_{1}} \\
c
\end{array}\right) \text { for all } c \in \mathbb{R}^{n-k_{1}}
$$


From (3.70), (3.75) and (3.73), it follows that

$$
\bar{y}^{z(c)}(n T)=\left(\hat{\mathcal{P}}_{\varepsilon}\right)^{n} z(c)=\left(\zeta_{1}, \ldots, \zeta_{k_{1}}\right) \tilde{A}_{12} c+\left(\hat{\eta}_{1}, \ldots, \hat{\eta}_{n-k_{1}}\right) \tilde{A}_{22} c \text { for all } c \in \mathbb{R}^{n-k_{1}} .
$$

Since $\left\{\zeta_{1}, \ldots, \zeta_{k_{1}}\right\}$ and $\left\{\hat{\eta}_{1}, \ldots, \hat{\eta}_{n-k_{1}}\right\}$ are accordingly the bases of $V$ and $V^{\perp}$, it holds that

$$
\left(\zeta_{1}, \ldots, \zeta_{k_{1}}\right) \tilde{A}_{12} c \in V \text { and }\left(\hat{\eta}_{1}, \ldots, \hat{\eta}_{n-k_{1}}\right) \tilde{A}_{22} c \in V^{\perp} \text { for all } c \in \mathbb{R}^{n-k_{1}}
$$

Let $\left(\bar{y}_{1}^{z(c)}(\cdot), \bar{y}_{2}^{z(c)}(\cdot)\right)$ be the unique decomposition of $\bar{y}^{z(c)}(\cdot)$ provided by Lemma 2.4, where $y(\cdot)$ and $u(\cdot)$ are replaced by $\bar{y}^{z(c)}(\cdot)$ and $\bar{u}^{z(c)}(\cdot)$ respectively. From the $T$-periodicity of the systems $(3.57),(2.14)$ and $(2.2)$, we see that

$$
\bar{y}_{1}^{z(c)}(n T) \in V \text { and } \bar{y}_{2}^{z(c)}(n T) \in V^{\perp} \text { for all } c \in \mathbb{R}^{n-k_{1}} .
$$

These, together with (3.76) and (3.77), indicate that

$$
\bar{y}_{2}^{z(c)}(n T)=\left(\hat{\eta}_{1}, \ldots, \hat{\eta}_{n-k_{1}}\right) \tilde{A}_{22} c \text { for all } c \in \mathbb{R}^{n-k_{1}} .
$$

Meanwhile, it follows from (2.15) and (3.74) that

$$
\bar{y}_{2}^{z(c)}(n T)=\bar{Q}^{\sim 1} \bar{Q} \mathcal{P}^{n} \bar{y}_{2}^{z(c)}(0)=\bar{Q}^{\sim 1} \bar{Q} \mathcal{P}^{n} z(c)=\bar{Q}^{\sim 1} \bar{Q}^{n}\left(\hat{\eta}_{1}, \ldots, \hat{\eta}_{n-k_{1}}\right) c \text { for all } c \in \mathbb{R}^{n-k_{1}} .
$$

From (3.78) and the above, we see that

$$
\left(\hat{\eta}_{1}, \ldots, \hat{\eta}_{n-k_{1}}\right) \tilde{A}_{22}=\bar{Q}^{\sim 1} \bar{Q} \mathcal{P}^{n}\left(\hat{\eta}_{1}, \ldots, \hat{\eta}_{n-k_{1}}\right) .
$$

On the other hand, because $V^{\perp}$ is invariant under $\bar{Q}^{\sim 1} \bar{Q} \mathcal{P}^{n}$ (see $\left.(2.15)\right)$, there is a matrix $\tilde{A}_{2} \in \mathbb{R}^{\left(n-k_{1}\right) \times\left(n-k_{1}\right)}$ such that

$$
\bar{Q}^{\sim 1} \bar{Q} \mathcal{P}^{n}\left(\hat{\eta}_{1}, \ldots, \hat{\eta}_{n-k_{1}}\right)=\left(\hat{\eta}_{1}, \ldots, \hat{\eta}_{n-k_{1}}\right) \tilde{A}_{2},
$$

and

$$
\sigma\left(\tilde{A}_{2}\right) \subseteq \sigma\left(\bar{Q}^{\sim 1} \bar{Q} \mathcal{P}^{n}\right)
$$

By (3.79) and (3.80), we find that

$$
\tilde{A}_{2}=\tilde{A}_{22}
$$

Since we already proved $(a) \Leftrightarrow \sigma\left(\bar{Q}^{\sim 1} \bar{Q}^{n}\right) \subset \mathcal{B}$ in Part 1 , and because we are in the case that $(a)$ is assumed to be true, it follows from (3.81) that

$$
\sigma\left(\tilde{A}_{2}\right) \subset \mathcal{B} .
$$

This, along with (3.82), leads to $\sigma\left(\tilde{A}_{22}\right) \subset \mathcal{B}$.

In summary, we conclude that the Proof of Theorem 1.1 is finished.

Remark 3.1. The periodic stabilization criterion for $T$-periodic pairs in Theorem 1.1 is an extension of Kalman's stabilization criterion for time-invariant pairs in $\mathbb{R}^{n \times n} \times \mathbb{R}^{n \times m}$. To see it, we let $[A, B] \in \mathbb{R}^{n \times n} \times \mathbb{R}^{n \times m}$, with the null-controllable subspace $V$. Write $\left\{\xi_{1}, \xi_{2}, \ldots, \xi_{k_{1}}\right\}$ and $\left\{\xi_{k_{1}+1}, \xi_{k_{1}+2}, \ldots, \xi_{n}\right\}$ for normalized orthogonal bases of $V$ and $V^{\perp}$, respectively. Let

$$
Q=\left(\xi_{1}, \ldots, \xi_{k_{1}}, \ldots, \xi_{n}\right) .
$$

By the classical linear control theory (see, for instance, Thm. 1.6, p. 110, [16]), there are matrices $A_{1} \in \mathbb{R}^{k_{1} \times k_{1}}$, $A_{2} \in \mathbb{R}^{k_{1} \times\left(n-k_{1}\right)}, A_{3} \in \mathbb{R}^{\left(n-k_{1}\right) \times\left(n-k_{1}\right)}$ and $B_{1} \in \mathbb{R}^{k_{1} \times m}$, with $\left[A_{1}, B_{1}\right]$ controllable, such that

$$
A=Q\left(\begin{array}{cc}
A_{1} & A_{2} \\
0 & A_{3}
\end{array}\right) Q^{*}, \quad B=Q\left(\begin{array}{c}
B_{1} \\
0
\end{array}\right)
$$


Furthermore, $[A, B]$ is stabilizable if and only if $A_{3}$ is exponentially stable; while $A_{3}$ is exponentially stable if and only if $[A, B]$ satisfies the Kalman's stabilization condition (see, for instance, [16] or [20]).

Let $\Phi(\cdot)$ be the fundamental solution associated with $A$, and $\bar{Q}$ be the matrix defined in Theorem 1.1, where $[A(\cdot), B(\cdot)]$ is replaced by $[A, B]$. By a direct calculation, we have that for any $T>0$,

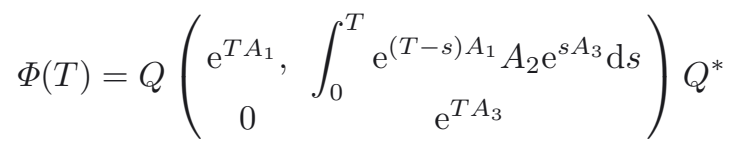

and

$$
\bar{Q}=Q\left(\begin{array}{cc}
0 & 0 \\
0 & \mathrm{e}^{T A_{3}^{*}} \mathrm{e}^{T A_{3}}
\end{array}\right) Q^{*}
$$

Thus, it holds that

$$
\bar{Q}^{\sim 1} \bar{Q} \Phi(T)=Q\left(\begin{array}{cc}
0 & 0 \\
0 & \mathrm{e}^{T A_{3}}
\end{array}\right) Q^{*}
$$

Hence, when $[A(\cdot), B(\cdot)] \equiv[A, B]$ is time-invariant, it holds that for any $T>0$

$$
\sigma\left(\bar{Q}^{\sim 1} \bar{Q} \Phi(T)\right) \subset \mathcal{B} \Leftrightarrow \sigma\left(\mathrm{e}^{T A_{3}}\right) \subset \mathcal{B} \Leftrightarrow \sigma\left(A_{3}\right) \subset \mathbb{C}^{-} \Leftrightarrow[A, B] \text { holds Kalman's stabilization condition . }
$$

From these, we see that if $[A(\cdot), B(\cdot)]=[A, B]$ is time-invariant, then (1.1) is $T$-periodically stabilizable for some $T>0$ if and only if (1.1) is $T$-periodically stabilizable for any $T>0$ if and only if (1.1) is feedback stabilizable by a constant matrix.

\section{The Proof of Theorems 1.2 And 1.3}

The Proof of Theorem 1.2. Let $\bar{Q}$ be the positive semi-definite matrix given by (1.7). By Lemma 2.3, it holds that $V=\mathcal{N}(\bar{Q})$. Then, there is an orthogonal matrix $P=\left(p_{1}, \ldots, p_{n}\right)$, with $p_{j}$ its $j$ th column vector, such that

$$
P^{*} \bar{Q} P=\operatorname{diag}\left\{\lambda_{1}, \ldots, \lambda_{n-k_{1}}, 0, \ldots, 0\right\},
$$

where $\lambda_{i}>0$ for $i=1, \ldots, n-k_{1}$ and $k_{1}$ is defined in (2.32). Hence,

$$
V=\operatorname{Span}\left\{p_{n-k_{1}+1}, \ldots, p_{n}\right\} .
$$

Along with the invariance of $V$ under $\mathcal{P}$, this indicates that

$$
\mathcal{P} P=P\left(\begin{array}{cc}
A_{1} & 0 \\
A_{2} & A_{3}
\end{array}\right)
$$

where $A_{1} \in \mathbb{R}^{\left(n-k_{1}\right) \times\left(n-k_{1}\right)}, A_{2} \in \mathbb{R}^{k_{1} \times\left(n-k_{1}\right)}, A_{3} \in \mathbb{R}^{k_{1} \times k_{1}}$. Hence,

$$
\begin{aligned}
& P^{*} \cdot \bar{Q}^{\sim 1} \bar{Q} \mathcal{P} \cdot P=\left(P^{*} \bar{Q}^{\sim 1} P\right)\left(P^{*} \bar{Q} P\right)\left(P^{*} \mathcal{P} P\right)=\left(P^{*} \bar{Q} P\right)^{\sim 1}\left(P^{*} \bar{Q} P\right)\left(P^{*} \mathcal{P} P\right) \\
= & \operatorname{diag}\left\{\lambda_{1}^{-1}, \ldots, \lambda_{n-k_{1}}^{-1}, 0, \ldots, 0\right\} \operatorname{diag}\left\{\lambda_{1}, \ldots, \lambda_{n-k_{1}}, 0, \ldots, 0\right\}\left(P^{*} \mathcal{P} P\right) \\
= & \left(\begin{array}{cc}
I_{n-k_{1}} & 0 \\
0 & 0_{k_{1} \times k_{1}}
\end{array}\right)\left(\begin{array}{cc}
A_{1} & 0 \\
A_{2} & A_{3}
\end{array}\right)=\left(\begin{array}{cc}
A_{1} & 0 \\
0 & 0
\end{array}\right) .
\end{aligned}
$$

From the above and the equivalence of $(b)$ and $(c)$ in Theorem 1.1, it follows that

(i) in Theorem $1.2 \Leftrightarrow \sigma\left(A_{1}\right) \subset \mathcal{B}$. 
Since $V$ is an invariant subspace of $\mathcal{P}$, we have (see Lem. 2.7 and Rem. 2.8)

$$
V=V_{1}(\mathcal{P}) \bigoplus V_{2}(\mathcal{P}), \sigma\left(\left.\mathcal{P}\right|_{V_{1}(\mathcal{P})}\right) \subset \mathcal{B}, \sigma\left(\left.\mathcal{P}\right|_{V_{2}(\mathcal{P})}\right) \subset \mathcal{B}^{c}
$$

From properties of $V_{2}(\mathcal{P})$ and $\mathbb{R}_{2}^{n}(\mathcal{P})$, one can easily check that

$$
\text { (ii) in Theorem } 1.2 \Leftrightarrow V_{1}(\mathcal{P})=\mathbb{R}_{2}^{n}(\mathcal{P}) \text {. }
$$

Thus, to prove (i) $\Leftrightarrow$ (ii), it suffices to verify

$$
V_{2}(\mathcal{P})=\mathbb{R}_{2}^{n}(\mathcal{P}) \Leftrightarrow \sigma\left(A_{1}\right) \subset \mathcal{B} .
$$

Now, we are on the position to prove (4.6). Write

$$
V_{1}(\mathcal{P})=\operatorname{Span}\left\{\eta_{1}, \ldots, \eta_{k_{2}}\right\} \text { with the basis }\left\{\eta_{1}, \ldots, \eta_{k_{2}}\right\}
$$

and

$$
V_{2}(\mathcal{P})=\operatorname{Span}\left\{\eta_{k_{2}+1}, \ldots, \eta_{k_{1}}\right\} \text { with the basis }\left\{\eta_{k_{2}+1}, \ldots, \eta_{k_{1}}\right\} .
$$

By (4.4), (4.7) and (4.8), there are invertible $A_{31} \in \mathbb{R}^{k_{2} \times k_{2}}$ and $A_{32} \in \mathbb{R}^{\left(k_{1}-k_{2}\right) \times\left(k_{1}-k_{2}\right)}$ such that

$$
\begin{gathered}
\mathcal{P}\left(\eta_{1}, \ldots, \eta_{k_{1}}\right)=\left(\eta_{1}, \ldots, \eta_{k_{1}}\right)\left(\begin{array}{cc}
A_{31} & 0 \\
0 & A_{32}
\end{array}\right) ; \\
\sigma\left(A_{31}\right)=\sigma\left(\left.\mathcal{P}\right|_{V_{1}(\mathcal{P})}\right) \subset \mathcal{B}, \quad \sigma\left(A_{32}\right)=\sigma\left(\left.\mathcal{P}\right|_{V_{2}(\mathcal{P})}\right) \subset \mathcal{B}^{c} .
\end{gathered}
$$

By (4.1), (4.7) and (4.8), $\left\{p_{1}, \ldots, p_{n-k_{1}}, \eta_{1}, \ldots, \eta_{k_{1}}\right\}$ is a basis of $\mathbb{R}^{n}$. From (4.2) and (4.9),

$$
\begin{aligned}
& \mathcal{P}\left(p_{1}, \ldots, p_{n-k_{1}}, \eta_{1}, \ldots, \eta_{k_{1}-k_{2}}, \ldots, \eta_{k_{1}}\right) \\
= & \left(p_{1}, \ldots, p_{n-k_{1}}, \eta_{1}, \ldots, \eta_{k_{1}-k_{2}}, \ldots, \eta_{k_{1}}\right)\left(\begin{array}{ccc}
A_{1} & 0 & 0 \\
A_{21} & A_{31} & 0 \\
A_{22} & 0 & A_{32}
\end{array}\right)
\end{aligned}
$$

for some $A_{21} \in \mathbb{R}^{k_{2} \times\left(n-k_{1}\right)}$ and $A_{22} \in \mathbb{R}^{\left(k_{1}-k_{2}\right) \times\left(n-k_{1}\right)}$. Write

$$
\begin{gathered}
\hat{A}_{1}=\left(\begin{array}{cc}
A_{1} & 0 \\
A_{21} & A_{31}
\end{array}\right) \in \mathbb{R}^{\left(n-k_{1}+k_{2}\right) \times\left(n-k_{1}+k_{2}\right)}, \\
\hat{A}_{2}=\left(A_{22}, 0_{\left(k_{1}-k_{2}\right) \times k_{2}}\right) \in \mathbb{R}^{\left(k_{1}-k_{2}\right) \times\left(n-k_{1}+k_{2}\right)}
\end{gathered}
$$

and

$$
\hat{P}=\left(p_{1}, \ldots, p_{n-k_{1}}, \eta_{1}, \ldots, \eta_{k_{1}}\right) .
$$

One can directly check that

$$
\hat{P}^{-1} \mathcal{P} \hat{P}=\left(\begin{array}{cc}
\hat{A}_{1} & 0 \\
\hat{A}_{2} & A_{32}
\end{array}\right) .
$$

The rest of the proof of (4.6) is carried out by the following two steps.

Step 1. $\sigma\left(A_{1}\right) \subset \mathcal{B} \Rightarrow V_{2}(\mathcal{P})=\mathbb{R}_{2}^{n}(\mathcal{P})$

Suppose that $\sigma\left(A_{1}\right) \subset \mathcal{B}$. Then, by (4.10) and (4.11), it holds that $\sigma\left(\hat{A}_{1}\right) \subset \mathcal{B}$. This, along with (4.10), yields that $\sigma\left(\hat{A}_{1}\right) \bigcap \sigma\left(A_{32}\right)=\varnothing$. Thus, the Sylvster equation

$$
A_{32} X-X \hat{A}_{1}=\hat{A}_{2}
$$


has a unique solution $\hat{X} \in \mathbb{R}^{\left(k_{1}-k_{2}\right) \times\left(n-k_{1}+k_{2}\right)}$ (see p. 131, [21]). Let

$$
\widetilde{P} \triangleq \hat{P}\left(\begin{array}{cc}
I_{n-k_{1}+k_{2}} & 0 \\
-\hat{X} & I_{k_{1}-k_{2}}
\end{array}\right) \text {. }
$$

From (4.13), (4.14) and (4.15), it follows that

$$
\widetilde{P}^{-1} \mathcal{P} \widetilde{P}=\left(\begin{array}{cc}
\hat{A}_{1} & 0 \\
0 & A_{32}
\end{array}\right)
$$

Write $\widetilde{p}_{j}$ for the $j$ th column vector of $\widetilde{P}$. Let

$$
Y_{1}=\operatorname{Span}\left\{\widetilde{p}_{1}, \widetilde{p}_{2}, \ldots, \widetilde{p}_{n-k_{1}+k_{2}}\right\} ; Y_{2}=\operatorname{Span}\left\{\widetilde{p}_{n-k_{1}+k_{2}+1}, \widetilde{p}_{n-k_{1}+k_{2}+2}, \ldots, \widetilde{p}_{n}\right\} .
$$

By (4.16), they are invariant subspaces of $\mathcal{P}$ and satisfy

$$
Y_{1} \oplus Y_{2}=\mathbb{R}^{n}, \sigma\left(\left.\mathcal{P}\right|_{Y_{1}}\right)=\sigma\left(\hat{A}_{1}\right) \subset \mathcal{B} \text { and } \sigma\left(\left.\mathcal{P}\right|_{Y_{2}}\right)=\sigma\left(A_{32}\right) \subset \mathcal{B}^{c} .
$$

Then, by Lemma 2.7, it stands that

$$
Y_{2}=\mathbb{R}_{2}^{n}(\mathcal{P})
$$

On the other hand, by (4.15) and (4.12),

$$
\widetilde{p}_{n-k_{1}+k_{2}+j}=\eta_{k_{2}+j}, \quad j=1, \ldots, k_{1}-k_{2} .
$$

Now, it follows from (4.18), (4.17), (4.19) and (4.8) that $\mathbb{R}_{2}^{n}(\mathcal{P})=V_{2}(\mathcal{P})$.

Step 2. $V_{2}(\mathcal{P})=\mathbb{R}_{2}^{n}(\mathcal{P}) \Rightarrow \sigma\left(A_{1}\right) \subset \mathcal{B}$

Suppose that

$$
V_{2}(\mathcal{P})=\mathbb{R}_{2}^{n}(\mathcal{P})
$$

Then it holds that

$$
k_{1}-k_{2}=n-k_{3},
$$

where $k_{3}$ is given by (2.32). By Lemma $(2.9), V_{1}(\mathcal{P}) \subseteq \mathbb{R}_{1}^{n}(\mathcal{P})$. Thus, we can write

$$
\mathbb{R}_{1}^{n}(\mathcal{P})=\operatorname{Span}\left\{\beta_{1}, \ldots, \beta_{n-k_{1}}, \eta_{1}, \ldots, \eta_{k_{2}}\right\} \text { with the basis }\left\{\beta_{1}, \ldots, \beta_{n-k_{1}}, \eta_{1}, \ldots, \eta_{k_{2}}\right\},
$$

where $\left\{\eta_{1}, \ldots, \eta_{k_{2}}\right\}$ is given by (4.7). By (4.20), we have

$$
\mathbb{R}_{1}^{n}(\mathcal{P}) \bigoplus V_{2}(\mathcal{P})=\mathbb{R}_{1}^{n}(\mathcal{P}) \bigoplus \mathbb{R}_{2}^{n}(\mathcal{P})=\mathbb{R}^{n}
$$

This, along with (4.22) and (4.8), yields that $\left\{\beta_{1}, \ldots, \beta_{n-k_{1}}, \eta_{1}, \ldots, \eta_{k_{1}}\right\}$ is a basis of $\mathbb{R}^{n}$. Recall that $\left\{p_{1}, \ldots, p_{n-k_{1}}, \eta_{1}, \ldots, \eta_{k_{1}}\right\}$ is also a basis of $\mathbb{R}^{n}$ (see (4.12)). Thus,

$$
\left(\beta_{1}, \ldots, \beta_{n-k_{1}}, \eta_{1}, \ldots, \eta_{k_{1}}\right)=\left(p_{1}, \ldots, p_{n-k_{1}}, \eta_{1}, \ldots, \eta_{k_{1}}\right)\left(\begin{array}{cc}
\hat{A}_{3} & 0 \\
\hat{A}_{4} & I_{k_{1}}
\end{array}\right),
$$

where $\hat{A}_{3} \in \mathbb{R}^{\left(n-k_{1}\right) \times k_{1}}$ is invertible and $\hat{A}_{4} \in \mathbb{R}^{k_{1} \times\left(n-k_{1}\right)}$. Since $\mathbb{R}_{1}^{n}(\mathcal{P})$ is invariant under $\mathcal{P}$ and $\sigma\left(\left.\mathcal{P}\right|_{\mathbb{R}_{1}^{n}(\mathcal{P})}\right) \subset \mathcal{B}$, by (4.9), there are $\hat{A}_{6} \in \mathbb{R}^{k_{2} \times\left(n-k_{1}\right)}$ and invertible $\hat{A}_{5} \in \mathbb{R}^{\left(n-k_{1}\right) \times\left(n-k_{1}\right)}$ with

$$
\sigma\left(\hat{A}_{5}\right) \subset \mathcal{B} .
$$


such that

$$
\begin{aligned}
& \mathcal{P}\left(\beta_{1}, \ldots, \beta_{n-k_{1}}, \eta_{1}, \ldots, \eta_{k_{2}}, \eta_{k_{2}+1} \ldots, \eta_{k_{1}}\right) \\
= & \left(\beta_{1}, \ldots, \beta_{k_{2}}, \eta_{1}, \ldots, \eta_{k_{2}}, \eta_{k_{2}+1} \ldots, \eta_{k_{1}}\right)\left(\begin{array}{ccc}
\hat{A}_{5} & 0 & 0 \\
\hat{A}_{6} & A_{31} & 0 \\
0 & 0 & A_{32}
\end{array}\right) .
\end{aligned}
$$

Write

$$
\hat{A}_{7}=\left(\begin{array}{c}
\hat{A}_{6} \\
0_{\left(k_{1}-k_{2}\right) \times\left(n-k_{1}\right)}
\end{array}\right) \in \mathbb{R}^{k_{1} \times\left(n-k_{1}\right)}, \quad \hat{A}_{8}=\left(\begin{array}{cc}
A_{31} & 0 \\
0 & A_{32}
\end{array}\right) \in \mathbb{R}^{k_{1} \times k_{1}} .
$$

From (4.12), (4.23), (4.25) and (4.26), we can directly check that

$$
\mathcal{P} \hat{P}=\hat{P}\left(\begin{array}{cc}
\hat{A}_{3} \hat{A}_{5} \hat{A}_{3}^{-1} & 0_{\left(n-k_{1}\right) \times k_{1}} \\
* & *
\end{array}\right) .
$$

Because both $\left\{p_{n-k_{1}+1}, \ldots, p_{n}\right\}$ and $\left\{\eta_{1}, \ldots, \eta_{k_{1}}\right\}$ are base of $V$ (see (4.1), (4.7) and (4.8)), there is an invertible matrix $A_{9} \in \mathbb{R}^{k_{1} \times k_{1}}$ such that $\hat{P}=P \cdot \operatorname{diag}\left\{I_{n-k_{1}}, A_{9}\right\}$. Then, it follows from (4.27) that

$$
P^{-1} \mathcal{P} P=\left(\begin{array}{cc}
\hat{A}_{3} \hat{A}_{5} \hat{A}_{3}^{-1} & 0 \\
* & *
\end{array}\right)
$$

Combined with (4.2), the above shows that $A_{1}=\hat{A}_{3} \hat{A}_{5} \hat{A}_{3}^{-1}$. Along with (4.24), this indicates that $\sigma\left(A_{1}\right)=$ $\sigma\left(\hat{A}_{5}\right) \subset \mathcal{B}$.

In summary, we complete the Proof of Theorem 1.2.

The Proof of Theorem 1.3. We organize the proof by the following two cases.

Case 1. where $V=\mathbb{R}^{n}$ : Let $y^{\varepsilon}(\cdot)$ be a solution to equation (1.3) with $K(\cdot)=K_{n}^{\varepsilon}(\cdot)$. It follows from the second equality in (2.9) that $\bar{Q}=0_{n \times n}$ in this case. This, together with (1.15), yields that $\bar{\rho}=+\infty$. Hence, the estimate (1.16) is equivalent to the estimate

$$
\left\|y^{\varepsilon}(t)\right\| \leq M \mathrm{e}^{-t / \delta}\left\|y^{\varepsilon}(0)\right\| \text { for any } t \in \mathbb{R}^{+} .
$$

By (1.7) and (3.13), we see that

$$
\lim _{\varepsilon \rightarrow 0+} \lambda_{1}(\varepsilon) \triangleq \lim _{\varepsilon \rightarrow 0+}\left\|\left(S_{n}^{\varepsilon}(0)\right)^{-1}-\bar{Q}\right\|^{\frac{1}{2}}=0 .
$$

Then, given $\delta>0$, there is an $\varepsilon \triangleq \varepsilon(\delta)>0$ such that

$$
\lambda_{1}(\varepsilon) \leq \mathrm{e}^{-n T / \delta} .
$$

Notice that any solution $y^{\varepsilon}(\cdot)$ to equation (1.3) with $K(\cdot)=K_{n}^{\varepsilon}(\cdot)$ satisfies that $y^{\varepsilon}(0) \in V$ in this case. Thus, it follows from (3.18), where $\lambda_{1}=\lambda_{1}(\varepsilon)$ (see (3.13)), and (4.29) that

$$
\left\|y^{\varepsilon}(j n T)\right\| \leq \mathrm{e}^{-j n T / \delta}\left\|y^{\varepsilon}(0)\right\| \text { for all } y^{\varepsilon}(0) \in V \text { and } j \in \mathbb{N} \text {. }
$$

Write $\Phi^{\varepsilon}(\cdot)$ for the fundamental solution associated with $A(\cdot)+B(\cdot) K_{n}^{\varepsilon}(\cdot)$. Let

$$
M_{1}^{\varepsilon} \triangleq \sup _{t \in[0, n T]}\left\|\Phi^{\varepsilon}(t)\left(\Phi^{\varepsilon}(n T)\right)^{-1}\right\| .
$$

By (4.31) and (4.30), we see that

$$
\begin{aligned}
\left\|y^{\varepsilon}(t)\right\| & =\left\|\Phi^{\varepsilon}(t)\left(\Phi^{\varepsilon}\left(\left[\frac{t}{n T}\right]+1\right) n T\right)^{-1} \Phi^{\varepsilon}\left(\left(\left[\frac{t}{n T}\right]+1\right) n T\right) y^{\varepsilon}(0)\right\| \\
& \leq M_{1}^{\varepsilon} \mathrm{e}^{-(n T / \delta)\left(\left[\frac{t}{n T}\right]+1\right)}\left\|y^{\varepsilon}(0)\right\| \leq M_{1}^{\varepsilon} \mathrm{e}^{-t / \delta}\left\|y^{\varepsilon}(0)\right\| \text { for each } t>0 .
\end{aligned}
$$

Since $(4.28) \Leftrightarrow(1.16)$ in this case, (4.32) leads to (1.16) in the case that $V=\mathbb{R}^{n}$. 
Case 2. where $V \neq \mathbb{R}^{n}$ : It follows from the second equality in (2.9) that $\bar{Q} \neq 0_{n \times n}$ for the current case. This implies that $\bar{Q}^{\sim 1} \bar{Q} \mathcal{P} \neq 0_{n \times n}$, i.e., there is a nonzero eigenvalue of $\bar{Q}^{\sim 1} \bar{Q} \mathcal{P}$. Thus, $\bar{\rho}<+\infty$ (see (1.15) for the definition of $\bar{\rho})$. Meanwhile, since $[A(\cdot), B(\cdot)]$ is $T$-periodically stabilizable, it follows from Theorem 1.1 that $\sigma\left(\bar{Q}^{\sim 1} \bar{Q} \mathcal{P}\right) \subset \mathcal{B}$. Hence, $\bar{\rho}>0($ see $(1.15))$.

Given $\delta>0$, we take

$$
\rho_{2}=\max \{\bar{\rho}-\delta \bar{\rho} / 2\} \text { and } \hat{\lambda}=\mathrm{e}^{-\rho_{2} T} .
$$

Since $0<\rho_{2}<\bar{\rho}$, it follows from (1.15) and the second property in (4.33) that

$$
\max \left\{|\lambda| \mid \lambda \in \sigma\left(\bar{Q}^{\sim 1} \bar{Q} \mathcal{P}\right)\right\}<\hat{\lambda}<1 .
$$

This, together with (3.33), implies that

$$
\max \left\{|\lambda| \mid \lambda \in \sigma\left(\bar{Q}^{\sim 1} \bar{Q} \mathcal{P}^{n}\right)\right\}<\hat{\lambda}^{n}<1,
$$

i.e., the spectral radius of $\bar{Q}^{\sim 1} \bar{Q} \mathcal{P}^{n}$ is less than 1. By the equivalent definition of spectral radius (see [23]), there is a $\bar{k} \in \mathbb{N}$ such that

$$
\lambda_{2} \triangleq\left\|\left(\bar{Q}^{\sim 1} \bar{Q}^{n} \mathcal{P}\right)^{\bar{k}}\right\| \leq \hat{\lambda}^{\bar{k} n}=\mathrm{e}^{-\rho_{2} \bar{k} n T}<1 .
$$

On the other hand, in Case 1, we already proved that for the $\delta$ given above, there is an $\varepsilon=\varepsilon(\delta)>0$ such that (4.30) stands. Let

$$
\rho_{1} \triangleq \max \left\{\rho_{2}+1,1 / \delta\right\} \text { and } \lambda_{1} \triangleq \mathrm{e}^{-\rho_{1} n T} .
$$

Then, by (4.30), with $j=1$, and by (4.35), we see that

$$
\left\|y^{\varepsilon}(n T)\right\| \leq \mathrm{e}^{-\rho_{1} n T}\left\|y^{\varepsilon}(0)\right\|=\lambda_{1}\left\|y^{\varepsilon}(0)\right\| \text { for all } y^{\varepsilon}(0) \in V .
$$

Notice that (4.36) and (4.34) correspond to accordingly (3.16) (with different $\lambda_{1}$ ) and (3.20)(with different $\lambda_{2}$ and $\bar{k}$ ).

Now, for the above-mentioned $\varepsilon=\varepsilon(\delta)$, let $y^{\varepsilon}(\cdot)$ be a solution to equation (3.14) (=equation (1.3) with $\left.K(\cdot)=K_{n}^{\varepsilon}(\cdot)\right)$. Set $y^{\varepsilon}(0)=x$. Write $\left(y_{1}(\cdot ; 0, x), y_{2}(\cdot ; 0, x)\right)$ for the decomposition of $y^{\varepsilon}(\cdot)$ provided by Lemma 2.4 . Let $\left(\alpha_{j}, \beta_{j}\right)$ be defined by $(3.21)$, i.e.,

$$
a_{j}=y_{1}(j \bar{k} n T ; 0, x) \text { and } \beta_{j}=y_{2}(j \bar{k} n T ; 0, x) \text {, for each } j \in\{0,1,2, \ldots\} .
$$

Then, from (4.36) and (4.34) (they correspond to according to (3.16) and (3.20)), following the same arguments as those in Step 2 of Part 1 in the Proof of Theorem 1.1 (noticing that $\Phi^{\varepsilon}(\cdot)$ here corresponds to $\Psi_{\varepsilon}(\cdot)$ there), we can reach the estimate (3.26) for the current case, i.e.,

$$
\left(\begin{array}{c}
\left\|\alpha_{j+1}\right\| \\
\left\|\beta_{j+1}\right\|
\end{array}\right) \leq\left(\begin{array}{cc}
\mathrm{e}^{-\rho_{1} \bar{k} n T} & \mathrm{e}^{-\rho_{2} \bar{k} n T}+\left\|\Phi^{\varepsilon}(\bar{k} n T)\right\| \\
0 & \mathrm{e}^{-\rho_{2} \bar{k} n T}
\end{array}\right)\left(\begin{array}{c}
\left\|\alpha_{j}\right\| \\
\left\|\beta_{j}\right\|
\end{array}\right), \quad \forall j=0,1, \ldots
$$

Here, we have used (4.34) and (4.35).

Since $P_{V}$ and $P_{V^{\perp}}$ stand for the orthogonal projections of $\mathbb{R}^{n}$ to $V$ and $V^{\perp}$, respectively, it follows from (4.37), the definition of $\left(y_{1}(\cdot ; 0, x), y_{2}(\cdot ; 0, x)\right)$ and Lemma 2.4 that

$$
\alpha_{j}=y_{1}^{\varepsilon}(j \bar{k} n T)=P_{V}\left(y^{\varepsilon}(j \bar{k} n T)\right), \beta_{j}=y_{2}^{\varepsilon}(j \bar{k} n T)=P_{V^{\perp}}\left(y^{\varepsilon}(j \bar{k} n T)\right), \quad \forall j=0,1, \ldots
$$

Let $M_{2}^{\varepsilon}=1+\left\|\Phi^{\varepsilon}(\bar{k} n T)\right\|$. Then, by (4.38), one can easily check that

$$
\left(\begin{array}{l}
\left\|\alpha_{j}\right\| \\
\left\|\beta_{j}\right\|
\end{array}\right) \leq\left(\begin{array}{cc}
\mathrm{e}^{-j \rho_{1} \bar{k} n T} & M_{2}^{\varepsilon} \sum_{l=0}^{j-1} \mathrm{e}^{-\left(l \rho_{1}+(j-1-l) \rho_{2}\right) \bar{k} n T} \\
0 & \mathrm{e}^{-j \rho_{2} \bar{k} n T}
\end{array}\right)\left(\begin{array}{l}
\left\|\alpha_{0}\right\| \\
\left\|\beta_{0}\right\|
\end{array}\right), \quad \forall j=1,2, \ldots
$$


Because $\rho_{1} \geq \rho_{2}+1$ (see (4.35)), it holds that for each $j=1,2, \ldots$,

$$
\begin{aligned}
& \sum_{l=0}^{j-1} \mathrm{e}^{-\left(l \rho_{1}+(j-1-l) \rho_{2}\right) \bar{k} n T}=\mathrm{e}^{-(j-1) \rho_{2} \bar{k} n T} \sum_{l=0}^{j-1} \mathrm{e}^{-l\left(\rho_{1}-\rho_{2}\right) \bar{k} n T} \\
\leq & \mathrm{e}^{-(j-1) \rho_{2} \bar{k} n T} \sum_{l=0}^{j-1} \mathrm{e}^{-l \bar{k} n T} \leq \mathrm{e}^{-(j-1) \rho_{2} \bar{k} n T} \sum_{l=0}^{\infty} \mathrm{e}^{-l \bar{k} n T}=\frac{\mathrm{e}^{\rho_{2} \bar{k} n T}}{1-\mathrm{e}^{-\bar{k} n T}} \mathrm{e}^{-j \rho_{2} \bar{k} n T} .
\end{aligned}
$$

Let

$$
M_{3}^{\varepsilon}=M_{2}^{\varepsilon} \frac{\mathrm{e}^{\rho_{2} \bar{k} n T}}{1-\mathrm{e}^{-\bar{k} n T}}
$$

It follows from (4.40) and (4.41) that

$$
\left\|y^{\varepsilon}(j \bar{k} n T)\right\| \leq\left\|\alpha_{j}\right\|+\left\|\beta_{j}\right\| \leq \mathrm{e}^{-\rho_{1} j \bar{k} n T}\left\|\alpha_{0}\right\|+\left(1+M_{3}^{\varepsilon}\right) \mathrm{e}^{-\rho_{2} j \bar{k} n T}\left\|\beta_{0}\right\|, \quad \forall j=1,2, \ldots
$$

By (4.42) and by the same argument in (4.32), there is a $M>0$ such that

$$
\left\|y^{\varepsilon}(t)\right\| \leq M\left(\mathrm{e}^{-\rho_{1} t}\left\|\alpha_{0}\right\|+\mathrm{e}^{-\rho_{2} t}\left\|\beta_{0}\right\|\right) .
$$

Because $\rho_{1} \geq \frac{1}{\delta}$ (see (4.35)) and $\rho_{2} \geq \bar{\rho}-\delta$ (see (4.33)), the estimate (4.43), as well as (4.39) with $j=0$, leads to $(1.16)$.

This completes the proof.

\section{AN EXAMPLE}

Now we present an example to illustrate the results given by Theorems 1.1 and 1.2. Consider the controlled system

$$
\frac{\mathrm{d}}{\mathrm{d} t}\left(\begin{array}{l}
y_{1} \\
y_{2} \\
y_{3}
\end{array}\right)=\left(\begin{array}{ccc}
0 & 0 & 0 \\
1 & 0 & 0 \\
0 & 0 & -1
\end{array}\right)\left(\begin{array}{l}
y_{1} \\
y_{2} \\
y_{3}
\end{array}\right)+\left(\begin{array}{c}
1 \\
\{t\} \\
0
\end{array}\right) u(t), \quad t \geq 0
$$

where $\{t\}$ denotes the decimal part of $t$. Clearly, (5.1) is a 1-periodic controlled system. The corresponding fundamental solution and the transformation over $T=1$ are accordingly

$$
\Phi(t)=\left(\begin{array}{ccc}
1 & 0 & 0 \\
t & 1 & 0 \\
0 & 0 & \mathrm{e}^{-t}
\end{array}\right), t \in[0,1] ; \text { and } \mathcal{P}=\Phi(1)=\left(\begin{array}{ccc}
1 & 0 & 0 \\
1 & 1 & 0 \\
0 & 0 & \mathrm{e}^{-1}
\end{array}\right) .
$$

One can easily check that in this case,

$$
V_{1}=\operatorname{Span}\left\{e_{1}\right\} ; \mathcal{P}^{-1} V_{1}=\operatorname{Span}\left\{e_{1}-e_{2}\right\} ; \mathcal{P}^{-2} V_{1}=\operatorname{Span}\left\{e_{1}-2 e_{2}\right\}
$$

These, along with (2.1), imply that

$$
V=V_{1}+\mathcal{P}^{-1} V_{1}+\mathcal{P}^{-2} V_{1}=\operatorname{Span}\left\{e_{1}, e_{2}\right\} .
$$

Hence, $V_{1}$ is a proper subspace of $V$ in this case. By a direct calculation, we have that

$$
\mathbb{R}_{1}^{3}(\mathcal{P})=\operatorname{Span}\left\{e_{3}\right\} \text { and } \mathbb{R}_{2}^{3}(\mathcal{P})=\operatorname{Span}\left\{e_{1}, e_{2}\right\} .
$$

Hence, $\mathbb{R}_{2}^{3}(\mathcal{P})=V$. Then, by Theorem 1.2 , System (5.1) is 1-periodically stabilizable. 
Meanwhile, System (1.5) in the current case reads

$$
\begin{aligned}
& \frac{\mathrm{d}}{\mathrm{d} t} S_{3}^{\varepsilon}(t) \triangleq \frac{\mathrm{d}}{\mathrm{d} t}\left(\begin{array}{lll}
s_{11} & s_{12} & s_{13} \\
s_{12} & s_{22} & s_{23} \\
s_{13} & s_{23} & s_{33}
\end{array}\right)(t)-\left(\begin{array}{ccc}
0 & 0 & 0 \\
1 & 0 & 0 \\
0 & 0 & -1
\end{array}\right)\left(\begin{array}{lll}
s_{11} & s_{12} & s_{13} \\
s_{12} & s_{22} & s_{23} \\
s_{13} & s_{23} & s_{33}
\end{array}\right)(t) \\
& -\left(\begin{array}{lll}
s_{11} & s_{12} & s_{13} \\
s_{12} & s_{22} & s_{23} \\
s_{13} & s_{23} & s_{33}
\end{array}\right)(t)\left(\begin{array}{ccc}
0 & 1 & 0 \\
0 & 0 & 0 \\
0 & 0 & -1
\end{array}\right)+\frac{1}{\varepsilon}\left(\begin{array}{ccc}
1 & t t\} & 0 \\
t t\} & t t\}^{2} & 0 \\
0 & 0 & 0
\end{array}\right)(t)=0, \quad t \in[0,3],
\end{aligned}
$$

with the initial condition $S_{3}^{\varepsilon}(0)=I_{3}$. Its solution is

$$
S_{3}^{\varepsilon}(\cdot)=S_{3,1}^{\varepsilon}(\cdot)+S_{3,2}^{\varepsilon}(\cdot)+S_{3,3}^{\varepsilon}(\cdot),
$$

where

$$
\begin{gathered}
S_{3,1}^{\varepsilon}(t)=\left(\begin{array}{ccc}
\left(1+\frac{1}{\varepsilon}\right)-\frac{1}{\varepsilon}(t-2) & -1+\left(1+\frac{1}{\varepsilon}\right)(t-2)-\frac{1}{\varepsilon}(t-2)^{2} & 0 \\
-1+\frac{\varepsilon+1}{\varepsilon}(t-2)-\frac{(t-2)^{2}}{\varepsilon} & 2-2(t-2)+\frac{\varepsilon+1}{\varepsilon}(t-2)^{2}-\frac{(t-2)^{3}}{\varepsilon} & 0 \\
0 & \mathrm{e}^{3-t}
\end{array}\right) \chi_{[2,3]}, \\
S_{3,2}^{\varepsilon}(t)=\left(\begin{array}{ccc}
\left(1+\frac{2}{\varepsilon}\right)-\frac{1}{\varepsilon}(t-1) & -\left(2+\frac{1}{\varepsilon}\right)+\left(1+\frac{2}{\varepsilon}\right)(t-1)-\frac{1}{\varepsilon}(t-1)^{2} & 0 \\
-\frac{2 \varepsilon+1}{\varepsilon}+\frac{\varepsilon+2}{\varepsilon}(t-1)-\frac{(t-1)^{2}}{\varepsilon} & \frac{5 \varepsilon+1}{\varepsilon}-\frac{4 \varepsilon+2}{\varepsilon}(t-1)+\frac{\varepsilon+2}{\varepsilon}(t-1)^{2}-\frac{(t-1)^{3}}{\varepsilon} & 0 \\
0 & 0 & \mathrm{e}^{3-t}
\end{array}\right) \chi_{[1,2)}
\end{gathered}
$$

and

$$
S_{3,3}^{\varepsilon}(t)=\left(\begin{array}{ccc}
\left(1+\frac{3}{\varepsilon}\right)-\frac{1}{\varepsilon} t & -\left(3+\frac{3}{\varepsilon}\right)+\left(1+\frac{3}{\varepsilon}\right) t-\frac{1}{\varepsilon} t^{2} & 0 \\
-\left(3+\frac{3}{\varepsilon}\right)+\left(1+\frac{3}{\varepsilon}\right) t-\frac{1}{\varepsilon} t^{2} & \left(10+\frac{5}{\varepsilon}\right)-\left(6+\frac{6}{\varepsilon}\right) t+\left(1+\frac{3}{\varepsilon}\right) t^{2}-\frac{1}{\varepsilon} t^{3} & 0 \\
0 & 0 & \mathrm{e}^{3-t}
\end{array}\right) \chi_{[0,1)} .
$$

From the above, one can directly check that

$$
\left(S_{3}^{\varepsilon}(0)\right)^{-1}=\left(\begin{array}{ccc}
\frac{5 \varepsilon+10 \varepsilon^{2}}{6+17 \varepsilon+\varepsilon^{2}} & \frac{3 \varepsilon+3 \varepsilon^{2}}{6+17 \varepsilon+\varepsilon^{2}} & 0 \\
\frac{3 \varepsilon+3 \varepsilon^{2}}{6+17 \varepsilon+\varepsilon^{2}} & \frac{3 \varepsilon+\varepsilon^{2}}{6+17 \varepsilon+\varepsilon^{2}} & 0 \\
0 & 0 & \mathrm{e}^{-3}
\end{array}\right)
$$

and the 3-periodic matrix-valued function given by (1.8) in the current case is

$$
K_{3}^{\varepsilon}(t)=-\frac{1}{\varepsilon}\left(K_{31}^{\varepsilon}(t), K_{32}^{\varepsilon}(t), 0\right), t \in[0,3) ; \quad K_{3}^{\varepsilon}(t)=K_{3}^{\varepsilon}(t+3), t \in \mathbb{R}^{+},
$$

where

$$
\begin{aligned}
& \left(K_{31}^{\varepsilon}(t), K_{32}^{\varepsilon}(t)\right) \\
& =-\chi_{[2,3)}(1, t-2)\left(\begin{array}{cc}
\left(1+\frac{1}{\varepsilon}\right)-\frac{1}{\varepsilon}(t-2) & -1+\left(1+\frac{1}{\varepsilon}\right)(t-2)-\frac{1}{\varepsilon}(t-2)^{2} \\
-1+\frac{\varepsilon+1}{\varepsilon}(t-2)-\frac{(t-2)^{2}}{\varepsilon} & 2-2(t-2)+\frac{\varepsilon+1}{\varepsilon}(t-2)^{2}-\frac{(t-2)^{3}}{\varepsilon}
\end{array}\right)^{-1} \\
& -\chi_{[1,2)}(1, t-1)\left(\begin{array}{cc}
\left(1+\frac{2}{\varepsilon}\right)-\frac{1}{\varepsilon}(t-1) & -\left(2+\frac{1}{\varepsilon}\right)+\left(1+\frac{2}{\varepsilon}\right)(t-1)-\frac{1}{\varepsilon}(t-1)^{2} \\
-\frac{2 \varepsilon+1}{\varepsilon}+\frac{\varepsilon+2}{\varepsilon}(t-1)-\frac{(t-1)^{2}}{\varepsilon} & \frac{5 \varepsilon+1}{\varepsilon}-\frac{4 \varepsilon+2}{\varepsilon}(t-1)+\frac{\varepsilon+2}{\varepsilon}(t-1)^{2}-\frac{(t-1)^{3}}{\varepsilon}
\end{array}\right) \\
& -\chi_{[0,1)}(1, t)\left(\begin{array}{rr}
\left(1+\frac{3}{\varepsilon}\right)-\frac{1}{\varepsilon} t & -\left(3+\frac{3}{\varepsilon}\right)+\left(1+\frac{3}{\varepsilon}\right) t-\frac{1}{\varepsilon} t^{2} \\
-\left(3+\frac{3}{\varepsilon}\right)+\left(1+\frac{3}{\varepsilon}\right) t-\frac{1}{\varepsilon} t^{2} & \left(10+\frac{5}{\varepsilon}\right)-\left(6+\frac{6}{\varepsilon}\right) t+\left(1+\frac{3}{\varepsilon}\right) t^{2}-\frac{1}{\varepsilon} t^{3}
\end{array}\right)^{-1} .
\end{aligned}
$$


By (5.3), we see that

$$
\bar{Q}=\operatorname{diag}\left\{0,0, \mathrm{e}^{-3}\right\}
$$

From (5.2) and (5.4), we see that $\sigma\left(\bar{Q}^{\sim 1} \bar{Q} \mathcal{P}\right)=\left\{0, \mathrm{e}^{-1}\right\} \subset \mathcal{B}$. Thus, by making use of Theorem 1.1, we also deduce that System (5.1) is 1-periodically stabilizable. By (5.3) and (5.4), one can directly check that

$$
\left\|\left(S_{3}^{\varepsilon}(0)\right)^{-1}-\bar{Q}\right\|=\frac{8+11 \varepsilon+\sqrt{40+108 \varepsilon+117 \varepsilon^{2}}}{12+34 \varepsilon+2 \varepsilon^{2}} \varepsilon .
$$

Let $\varepsilon_{0}=(9+\sqrt{321}) / 20$. Then it follows from (5.5) that

$$
\left\|\left(S_{3}^{\varepsilon}(0)\right)^{-1}-\bar{Q}\right\| \leq \frac{8 \varepsilon+11 \varepsilon^{2}}{6+17 \varepsilon+\varepsilon^{2}}<1 \text { for each } \varepsilon \in\left(0, \varepsilon_{0}\right) .
$$

Now, by Theorem 1.1 and (5.6), each $K_{3}^{\varepsilon}(\cdot)$, with $\varepsilon \in\left(0, \varepsilon_{0}\right)$, is a 3-periodic feedback stabilization law for system (5.1).

To construct a 1-periodic feedback stabilization law for system (5.1), we can use the method in the Proof of Theorem 1.1 (see Step 1 in Part 2 in the proof) to get

$$
X=\left(\begin{array}{ccc}
1 & 1 & 0 \\
0 & -1 & 0 \\
0 & 0 & 1
\end{array}\right)
$$

Thus, the solution to equation (1.6) corresponding to the above-mentioned $X$ is

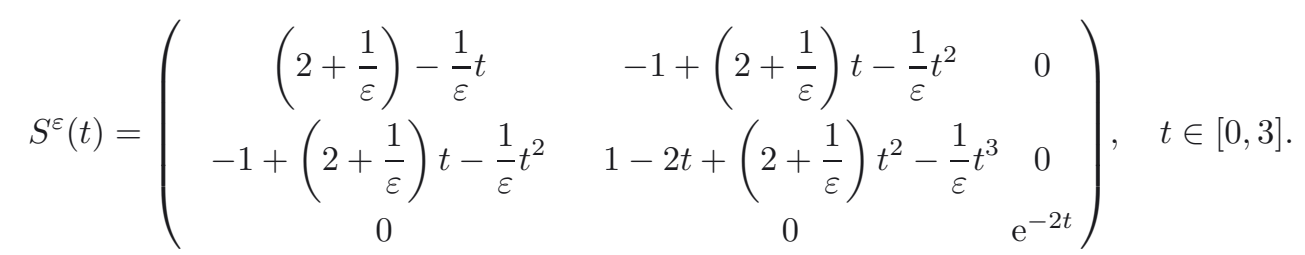

Then, by a direct calculation, the matrix-valued function given by (1.9) in the current case is

$$
K_{1}^{\varepsilon}(t)=\frac{-1}{(1-t)+\varepsilon}(1-t, 1,0), \quad t \in[0,1) ; \quad K_{1}^{\varepsilon}(t)=K_{1}^{\varepsilon}(t+1), t \in \mathbb{R}^{+} .
$$

Let $\varepsilon_{0}$ be given by (3.55) where $X$ is given by (5.7). (We omit the detail computation for this $\varepsilon_{0}$.) Then, from the Proof of Theorem 1.1 (see Step 3 in Part 2 of the proof), as well as $(3.55)$, each $K_{1}^{\varepsilon}(\cdot)$ with $\varepsilon \in\left(0, \varepsilon_{0}\right)$ is a 1-periodic stabilization feedback law for system (5.1).

\section{An applichtion of Theorem 1.2}

In this section, we will apply Theorem 1.2 to find a way to determine $M\left(\mathcal{C B}_{A(\cdot)}\right)$ given by (1.18) and to design a $\hat{B} \in \mathcal{C B}_{A(\cdot)}$ with $M\left(\mathcal{C B}_{A(\cdot)}\right)$ columns, for a given $T$-periodic $A(\cdot)$ in $L^{\infty}\left(\mathbb{R}^{+} ; \mathbb{R}^{n}\right)$. Here, $\mathcal{C} \mathcal{B}_{A(\cdot)}$ is given by (1.17).

\subsection{The case where $A(\cdot)$ is time-varying}

The following proposition is a consequence of Theorem 1.2.

Proposition 6.1. Let $[A(\cdot), B(\cdot)]$ be a T-periodic pair with $V$ and $\mathcal{P}$ given by (1.10) and (1.4), respectively. Then, the following statements are equivalent:

(a) the pair $[A(\cdot), B(\cdot)]$ is T-periodically stabilizable; 
(b) the property (1.14) stands;

(c) it holds that

$$
V+\mathbb{R}_{1}^{n}(\mathcal{P})=\mathbb{R}^{n}
$$

(d) it holds that

$$
V_{2}(\mathcal{P})=\mathbb{R}_{2}^{n}(\mathcal{P}) .
$$

Here, $V_{2}(\mathcal{P}), \mathbb{R}_{2}^{n}(\mathcal{P})$ and $\mathbb{R}_{1}^{n}(\mathcal{P})$ are given by (1.12).

Proof. First, it follows from Theorem 1.2 that $(a) \Leftrightarrow(b)$.

Next, we suppose that $(6.1)$ stands. Since $V=V_{1}(\mathcal{P}) \oplus V_{2}(\mathcal{P})$, we have

$$
\left(V_{1}(\mathcal{P})+\mathbb{R}_{1}^{n}(\mathcal{P})\right)+V_{2}(\mathcal{P})=\mathbb{R}^{n}
$$

It follows from Lemma 2.9 that $V_{1}(\mathcal{P})+\mathbb{R}_{1}^{n}(\mathcal{P})=\mathbb{R}_{1}^{n}(\mathcal{P})$ and $V_{2}(\mathcal{P}) \subseteq \mathbb{R}_{2}^{n}(\mathcal{P})$. Thus (6.2) holds. Since $V=V_{1}(\mathcal{P}) \bigoplus V_{2}(\mathcal{P})$, from $(6.2)$, we get $(1.14)$. Hence, $(c) \Rightarrow(d) \Rightarrow(b)$.

Finally, it follows from (1.14) that

$$
\mathbb{R}^{n} \supseteq V+\mathbb{R}_{1}^{n}(\mathcal{P}) \supseteq \mathbb{R}_{2}^{n}(\mathcal{P})+\mathbb{R}_{1}^{n}(\mathcal{P})=\mathbb{R}^{n},
$$

which leads to (6.1), i.e., $(b) \Rightarrow(c)$. This completes the proof.

For each $T$-periodic $A(\cdot) \in L^{\infty}\left(\mathbb{R}^{+} ; \mathbb{R}^{n}\right)$ with $\mathcal{P}$ given by (1.4), one can structure an invertible matrix $Q$ such that

$$
Q^{-1} \mathcal{P} Q=\left(\begin{array}{ll}
\mathcal{P}_{1} & \\
& \mathcal{P}_{2}
\end{array}\right)
$$

where $\mathcal{P}_{1}$ and $\mathcal{P}_{2}$ are real square matrices with $\sigma\left(\mathcal{P}_{1}\right)$ in $\mathcal{B}$ and $\sigma\left(\mathcal{P}_{2}\right)$ in $\mathcal{B}^{c}$ respectively. Write $q_{j}$ for the $j$ th column vector in $Q$. Then, by (2.32),

$$
k_{3} \triangleq \operatorname{dim}\left(\mathbb{R}_{1}^{n}(\mathcal{P})\right)=\operatorname{dim}\left(\mathcal{P}_{1}\right)
$$

and

$$
\mathbb{R}_{1}^{n}(\mathcal{P})=\operatorname{Span}\left\{q_{1}, \ldots, q_{k_{3}}\right\} .
$$

The next Corollary 6.2 is a consequence of Proposition 6.1.

Corollary 6.2. Let $[A(\cdot), B(\cdot)]$ be a T-periodic pair, with $V, \mathcal{P}, Q$ and $k_{3}$ given by (1.10), (1.4), (6.4) and (6.5), respectively. Then, $[A(\cdot), B(\cdot)]$ is T-periodically stabilizable if and only if

$$
\left(0_{\left(n-k_{3}\right) \times k_{3}}, I_{n-k_{3}}\right) \quad Q^{-1} V=\mathbb{R}^{n-k_{3}} .
$$

Proof. By (2.32), we have $\operatorname{dim} V=k_{1}$. Thus, we can write

$$
V=\operatorname{Span}\left\{\eta_{1}, \eta_{2}, \ldots, \eta_{k_{1}}\right\} \text { with the basis }\left\{\eta_{1}, \eta_{2}, \ldots, \eta_{k_{1}}\right\} .
$$

Clearly, there are matrix $C_{1} \in \mathbb{R}^{k_{3} \times k_{1}}, C_{2} \in \mathbb{R}^{\left(n-k_{3}\right) \times k_{1}}$ such that

$$
\left(q_{1}, q_{2}, \ldots, q_{k_{3}}, \eta_{1}, \eta_{2}, \ldots, \eta_{k_{1}}\right)=\left(q_{1}, q_{2}, \ldots, q_{k_{3}}, q_{k_{3}+1}, \ldots, q_{n}\right)\left(\begin{array}{cc}
I_{k_{3}} & C_{1} \\
& C_{2}
\end{array}\right) .
$$

From (6.8) and (6.9),

$$
\begin{aligned}
& \left(0_{\left(n-k_{3}\right) \times k_{3}}, I_{n-k_{3}}\right) Q^{-1} V=\left(0_{\left(n-k_{3}\right) \times k_{3}}, I_{n-k_{3}}\right) Q^{-1} \operatorname{Span}\left\{\eta_{1}, \eta_{2}, \ldots, \eta_{k_{1}}\right\} \\
= & \mathcal{R}\left(\left(0_{\left(n-k_{3}\right) \times k_{3}}, I_{n-k_{3}}\right) Q^{-1}\left(\eta_{1}, \eta_{2}, \ldots, \eta_{k_{1}}\right)\right) \\
= & \mathcal{R}\left(\left(0_{\left(n-k_{3}\right) \times k_{3}}, I_{n-k_{3}}\right)\left(\begin{array}{l}
C_{1} \\
C_{2}
\end{array}\right)\right)=\mathcal{R}\left(C_{2}\right) .
\end{aligned}
$$


Since $\left\{q_{1}, \ldots, q_{n}\right\}$ is a basis of $\mathbb{R}^{n}$, from Proposition 6.1, (6.6), (6.8), (6.9) and (6.10), we have

$$
\begin{aligned}
& \mathbb{R}_{u}^{n}(\mathcal{P}) \subseteq V \Leftrightarrow V+\left(\mathbb{R}^{n}\right)_{\mathcal{P}}=\mathbb{R}^{n} \\
\Leftrightarrow & \operatorname{Span}\left\{q_{1}, q_{2}, \ldots, q_{k_{3}}, \eta_{1}, \eta_{2}, \ldots, \eta_{k_{1}}\right\}=\mathbb{R}^{n} \\
\Leftrightarrow & \mathcal{R}\left(\left(\begin{array}{r}
I_{k_{3}} \\
C_{1} \\
C_{2}
\end{array}\right)\right)=\mathbb{R}^{n} \Leftrightarrow \mathcal{R}\left(C_{2}\right)=\mathbb{R}^{n-k_{3}} \\
\Leftrightarrow & \left(0_{\left(n-k_{3}\right) \times k_{3}}, I_{n-k_{3}}\right) Q^{-1} V=\mathbb{R}^{n-k_{3}} .
\end{aligned}
$$

From (6.11) and Proposition 6.1, it follows that $[A(\cdot), B(\cdot)]$ is T-periodically stabilizable if and only if $(6.7)$ holds. This completes the proof.

Proposition 6.3. For each $T$-periodic $A(\cdot)$ in $L^{\infty}\left(\mathbb{R}^{+} ; \mathbb{R}^{n}\right)$, the $n \times n$ identity matrix $I_{n}$ belongs to $\mathcal{C B}_{A(\cdot)}$. Consequently, $\mathcal{C B}_{A(\cdot)} \neq \varnothing$ and $M\left(\mathcal{C B}_{A(\cdot)}\right) \leq n$.

Proof. By Theorem 1.2, it suffices to show that $\left[A(\cdot), I_{n}\right]$ is null controllable over $[0, T]$. It is well-known that the later is equivalent to the non-singularity of the Gramian

$$
G \triangleq \int_{0}^{T} \Phi^{-1}(t) \Phi^{-*}(t) \mathrm{d} t
$$

which is clearly invertible in this case. This completes the proof.

In what follows, we arbitrarily fix a $T$-periodic $A(\cdot) \in L^{\infty}\left(\mathbb{R}^{+} ; \mathbb{R}^{n}\right)$ with $\mathcal{P}, Q$ and $k_{3}$ given by (1.4), (6.4) and (6.5), respectively. Let

$$
\Phi([0, n T]) \triangleq \operatorname{Span}\left\{\Phi^{-1}(s) \mid s \in[0, n T]\right\} \text { and } \bar{k} \triangleq \operatorname{dim}(\Phi([0, n T])) .
$$

Here, $\Phi(\cdot)$ is the fundamental solution associated with $A(\cdot)$. The following proposition provides a way to determine $\bar{k}$.

Proposition 6.4. It holds that

$$
\operatorname{dim}(\Phi([0, n T])) \triangleq \bar{k}=\operatorname{rank}\left(\int_{0}^{n T} \operatorname{vec}\left(\Phi^{-1}(t)\right)\left(\operatorname{vec}\left(\Phi^{-1}(t)\right)\right)^{*} \mathrm{~d} t\right),
$$

where vec(.): $\mathbb{R}^{n \times n} \mapsto \mathbb{R}^{n^{2} \times 1}$ is defined by

$$
\operatorname{vec}(D)=\left(d_{11}, \ldots, d_{n 1}, d_{12}, \ldots, d_{n 2}, \ldots, d_{1 n}, \ldots, d_{n n}\right)^{*} \text { for each } D=\left(d_{i j}\right)_{n \times n} .
$$

Proof. Let $\left\{\Phi_{1}, \ldots, \Phi_{\bar{k}}\right\}$ satisfy that $\operatorname{Span}\left\{\Phi_{1}, \ldots, \Phi_{\bar{k}}\right\}=\operatorname{Span}\left\{\Phi^{-1}(s) \mid s \in[0, n T]\right\}$. By the continuity of $\Phi^{-1}(\cdot)$, there is a $\mathbb{R}^{\bar{k}}$-valued continuous functions $c(\cdot)=\left(c_{1}(\cdot), c_{2}(\cdot), \ldots, c_{\bar{k}}(\cdot)\right)^{*}$ such that

$$
\operatorname{vec}\left(\Phi^{-1}(s)\right)=\sum_{j=1}^{\bar{k}} c_{j}(s) \operatorname{vec}\left(\Phi_{j}\right)=\left(\operatorname{vec}\left(\Phi_{1}\right), \ldots, \operatorname{vec}\left(\Phi_{\bar{k}}\right)\right) c(s) .
$$

By (6.14), one can easily check that

$$
\int_{0}^{n T} \operatorname{vec}\left(\Phi^{-1}(s)\right)\left(\operatorname{vec}\left(\Phi^{-1}(s)\right)\right)^{*} \mathrm{~d} s=\left(\operatorname{vec}\left(\Phi_{1}\right), \ldots, \operatorname{vec}\left(\Phi_{\bar{k}}\right)\right) \int_{0}^{n T} c(s) c(s)^{*} \mathrm{~d} s\left(\operatorname{vec}\left(\Phi_{1}\right), \ldots, \operatorname{vec}\left(\Phi_{\bar{k}}\right)\right)^{*} .
$$

This implies

$$
\operatorname{rank}\left(\int_{0}^{n T} \operatorname{vec}\left(\Phi^{-1}(s)\right)\left(\operatorname{vec}\left(\Phi^{-1}(s)\right)\right)^{*} \mathrm{~d} s\right)=\operatorname{rank}\left(\int_{0}^{n T} c(s) c(s)^{*} \mathrm{~d} s\right) \leq \bar{k}
$$


The rest is to show the non-singularity of the $\bar{k} \times \bar{k}$ matrix $\left(\int_{0}^{n T} c(s) c(s)^{*} \mathrm{~d} s\right)$. In fact, when $\xi \in \mathbb{R}^{\bar{k}}$ is such that $\xi^{*}\left(\int_{0}^{n T} c(s) c^{*}(s) \mathrm{d} s\right) \xi=0$, we have

$$
c(s)^{*} \xi \equiv 0 \text { for all } s \in[0, n T] .
$$

On the other hand, because of $(6.12)$, there are $s_{1}, \ldots, s_{\bar{k}} \in[0, n T]$ such that

$$
\left(\operatorname{vec}\left(\Phi^{-1}\left(s_{1}\right)\right), \ldots, \operatorname{vec}\left(\Phi^{-1}\left(s_{\bar{k}}\right)\right)\right)=\left(\operatorname{vec}\left(\Phi_{1}\right), \ldots, \operatorname{vec}\left(\Phi_{\bar{k}}\right)\right) C,
$$

where $C$ is an invertible $\bar{k} \times \bar{k}$ real matrix, and such that

$$
\operatorname{vec}\left(\Phi^{-1}(s)\right)=\left(\operatorname{vec}\left(\Phi^{-1}\left(s_{1}\right)\right), \ldots, \operatorname{vec}\left(\Phi^{-1}\left(s_{\bar{k}}\right)\right)\right) \widetilde{c}(s) \text { for each } s \in[0, n T]
$$

where $\widetilde{c}(s)=\left(\widetilde{c}_{1}(s), \ldots, \widetilde{c}_{\bar{k}}(s)\right)^{*}$, with $\widetilde{c}_{i}(\cdot)$ a function from $[0, n T]$ to $\mathbb{R}$. It follows from $(6.14),(6.16)$ and $(6.17)$ that

$$
c(s)=C \widetilde{c}(s) \text { for each } s \in[0, n T]
$$

From (6.17), we also have

$$
\widetilde{c}\left(s_{j}\right)=e_{j} \text { for all } j=1, \ldots, \bar{k},
$$

where $\left\{e_{1}, \ldots, e_{\bar{k}}\right\}$ is the standard basis of $\mathbb{R}^{\bar{k}}$. finally, it follows from (6.19), (6.18) and (6.15) that

$$
C^{*} \xi=I_{\bar{k}} C^{*} \xi=\left(\begin{array}{c}
\widetilde{c}\left(s_{1}\right)^{*} \\
\cdots \\
\widetilde{c}\left(s_{\bar{k}}\right)^{*}
\end{array}\right) C^{*} \xi=\left(\begin{array}{c}
\widetilde{c}\left(s_{1}\right)^{*} C^{*} \\
\cdots \\
\widetilde{c}\left(s_{\bar{k}}\right)^{*} C^{*}
\end{array}\right) \xi=\left(\begin{array}{c}
c\left(s_{1}\right)^{*} \\
\ldots \\
c\left(s_{\bar{k}}\right)^{*}
\end{array}\right) \xi=0 .
$$

which together with the non-singularity of $C$, implies that $\xi=0$. This completes the proof.

Definition 6.5. A family of matrices $\left\{\Phi_{i}\right\}_{i=1}^{\hat{k}}$ in $\mathbb{R}^{n \times n}$, with $\hat{k} \geq \bar{k}$, is called a family generating $\Phi([0, n T])$ if

$$
\operatorname{Span}\left\{\Phi_{1}, \ldots, \Phi_{\hat{k}}\right\}=\operatorname{Span}\left\{\Phi^{-1}(s) \mid s \in[0, n T]\right\} .
$$

In general, a family generating $\Phi([0, n T])$ is not necessarily linearly independent. When $\hat{k}=\bar{k}$, it is a linearly independent family.

Definition 6.6. Let $\left\{\Phi_{i}\right\}_{i=1}^{\hat{k}}$ be a family generating $\Phi([0, n T])$. A family of matrices $\left\{\bar{A}_{i}\right\}_{i=1}^{\hat{k}}$ is called the family affiliated to $\left\{\Phi_{i}\right\}_{i=1}^{\hat{k}}$ if

$$
\bar{A}_{i}=\left[\left(0_{\left(n-k_{3}\right) \times k_{3}}, I_{n-k_{3}}\right) Q^{-1} \Phi_{i}\right] \in \mathbb{R}^{\left(n-k_{3}\right) \times n} \text { for each } i=1, \ldots, \hat{k},
$$

where $Q$ is given by (6.4).

Lemma 6.7. Let $B \in \mathbb{R}^{n \times m}$. Let $\left\{\Phi_{i}\right\}_{i=1}^{\hat{k}}$ be a family generating $\Phi([0, n T])$. Then

$$
V_{[A(\cdot), B]}=\operatorname{Span}\left\{\Phi_{i} B v \mid 1 \leq i \leq \hat{k}, v \in \mathbb{R}^{m}\right\} .
$$


Proof. By (6.20), it suffices to show

$$
V_{[A(\cdot), B]}=\operatorname{Span}\left\{\Phi^{-1}(s) B v \mid s \in[0, n T], v \in \mathbb{R}^{m}\right\} .
$$

From (2.1) and (2.3) in Lemma 2.1,

$$
V_{[A(\cdot), B]}=\left\{\int_{0}^{n T} \Phi^{-1}(t) B u(t) \mathrm{d} t \mid u(\cdot) \in L^{2}\left(0, n T ; \mathbb{R}^{m}\right)\right\} .
$$

For each $s \in[0, n T)$ and $v \in \mathbb{R}^{m}$, we take

$$
x^{\varepsilon} \triangleq \frac{1}{\varepsilon} \int_{0}^{n T} \Phi^{-1}(t) B \chi_{[s, s+\varepsilon]}(t) v \mathrm{~d} t,
$$

where $\varepsilon \in(0, n T-s)$. By $(6.24), x^{\varepsilon} \in V_{[A(\cdot), B]}$. Sending $\varepsilon \rightarrow 0$, we get

$$
\Phi^{-1}(s) B v=\lim _{\varepsilon \rightarrow 0} x^{\varepsilon} \in V_{[A(\cdot), B]},
$$

since $V_{[A(\cdot), B]}$ is closed. Similarly, we can prove that

$$
\Phi^{-1}(n T) B v \in V_{[A(\cdot), B]} \text { for each } v \in \mathbb{R}^{m} .
$$

Hence, the space on the right hand side of (6.23) is a subspace of $V_{[A(\cdot), B]}$.

Conversely, for each $u(\cdot) \in L^{2}\left(0, n T ; \mathbb{R}^{m}\right)$, there is a sequence of step functions on $[0, n T]$, denoted by $\left\{u_{k}(\cdot)\right\}$, such that $u_{k}(\cdot)$ converges to $u(\cdot)$ in $L^{2}\left(0, n T ; \mathbb{R}^{m}\right)$. One can easily check that each $\int_{0}^{n T} \Phi^{-1}(t) B u_{k}(t) \mathrm{d} t$ belongs to the space on the right hand side of (6.23). Thus,

$$
\int_{0}^{n T} \Phi^{-1}(t) B u(t) \mathrm{d} t=\lim _{k \rightarrow \infty} \int_{0}^{n T} \Phi^{-1}(t) B u_{k}(t) \mathrm{d} t
$$

is in the space on the right side of (6.23), which, along with (6.24), indicates that $V_{[A(\cdot), B]}$ is a subset of the space on the right side of (6.23). This completes the proof.

Proposition 6.8. Let $\left\{\Phi_{i}\right\}_{i=1}^{\hat{k}}$ be a family generating $\Phi([0, n T])$, with its affiliated family $\left\{\bar{A}_{i}\right\}_{i=1}^{\hat{k}}$ (see (6.21)). Then, it holds that

$$
B \in \mathcal{C B}_{A(\cdot)} \Leftrightarrow \sum_{i=1}^{\hat{k}} \bar{A}_{i} \mathcal{R}(B)=\mathbb{R}^{n-k_{3}} .
$$

Proof. From (1.17) and Corollary 6.2, we see that $B \in \mathcal{C B}_{A(\cdot)} \Leftrightarrow(6.7)$. By Lemma 6.7, (6.7) is equivalent to

$$
\left(0_{\left(n-k_{3}\right) \times k_{3}}, I_{n-k_{3}}\right) Q^{-1} \operatorname{Span}\left\{\Phi_{i} B v \mid 1 \leq i \leq \hat{k}, v \in \mathbb{R}^{m}\right\}=\mathbb{R}^{n-k_{3}} .
$$

By (6.21), the left side of (6.26) is the same as the space $\operatorname{Span}\left\{A_{i} B v \mid 1 \leq i \leq \hat{k}, v \in \mathbb{R}^{m}\right\}$. Meanwhile, it is clear that

Hence, it holds that

$$
\sum_{i=1}^{\hat{k}} \bar{A}_{i} \mathcal{R}(B)=\operatorname{Span}\left\{\bar{A}_{i} B v \mid 1 \leq i \leq \hat{k}, v \in \mathbb{R}^{n}\right\} .
$$

$$
\sum_{i=1}^{\hat{k}} \bar{A}_{i} \mathcal{R}(B)=\left(0_{\left(n-k_{3}\right) \times k_{3}}, I_{n-k_{3}}\right) \quad Q^{-1} \operatorname{Span}\left\{\Phi_{i} B v \mid 1 \leq i \leq \hat{k}, v \in \mathbb{R}^{m}\right\} .
$$

Thus, (6.26) is equivalent to $\sum_{i=1}^{\hat{k}} \bar{A}_{i} \mathcal{R}(B)=\mathbb{R}^{n-k_{3}}$. This completes the proof.

Next, we introduce the concept of symbol matrices. 
Definition 6.9. Let $\alpha$ be a $l$-dimensional vector variable, i.e., $\alpha=\left(x_{1}, \ldots, x_{l}\right)^{*}$, where $x_{1}, \ldots, x_{l}$ are variables in $\mathbb{R}$. A symbol matrix with respect to $\alpha$ is a matrix whose elements are linear functions of $\alpha$.

By the definition, when $\alpha$ is a $l$-dimensional vector variable, a $n^{\prime} \times m^{\prime}$ symbol matrix w.r.t. $\alpha$ can be expressed as:

$$
\mathcal{A}(\alpha)=\left(\mathcal{A}(\alpha)_{i j}\right)_{i=1, j=1}^{i=n^{\prime}, j=m^{\prime}} \text {, with } \mathcal{A}(\alpha)_{i j}=<\overrightarrow{a_{i j}}, \alpha>_{\mathbb{R}^{l}} \text { for some } \overrightarrow{a_{i j}} \in \mathbb{R}^{l}
$$

Definition 6.10. Let $\alpha$ be a $l$-dimensional vector variable and $\mathcal{A}(\alpha)$ be a $n^{\prime} \times m^{\prime}$ symbol matrix w.r.t. $\alpha$. A non-negative integer $j$ is called the rank of $\mathcal{A}(\alpha)$, if the following conditions hold

(i) any $(j+1)$-order sub-determinant of $\mathcal{A}(\alpha)$ is identically zero;

(ii) there is an $\bar{\alpha} \in \mathbb{R}^{l}$ such that some $j$-order sub-determinant of the matrix $\mathcal{A}(\bar{\alpha})$ is not zero.

The rank of $\mathcal{A}(\alpha)$ is denoted by $\operatorname{Rank}(\mathcal{A}(\alpha))$.

When $\alpha=\left(x_{1}, \ldots, x_{n}\right)^{*}$ is a $n$-dimensional vector variable, and $D=\left(d_{l k}\right) \in \mathbb{R}^{m^{\prime} \times n}$ with $m^{\prime} \in \mathbb{N}$, we define

$$
D \alpha=\left(\sum_{k=1}^{n} d_{1, k} x_{k}, \ldots, \sum_{k=1}^{n} d_{m_{1}^{\prime}, k} x_{k}\right)^{*} .
$$

In what follows, we give $n$-dimensional vector variables $\alpha_{1}, \alpha_{2}, \ldots, \alpha_{n}$, with $\alpha_{i}=\left(x_{1}^{i}, \ldots, x_{n}^{i}\right)^{*}$ for $i=1, \ldots, n$. We define

$$
\left(\alpha_{1}, \alpha_{2}, \ldots, \alpha_{j}\right)=\left(x_{1}^{1}, \ldots, x_{n}^{1}, x_{1}^{2}, \ldots, x_{n}^{2} \ldots, x_{1}^{j}, \ldots, x_{n}^{j}\right)^{*} \text { for each } 1 \leq j \leq n .
$$

Clearly, it is a $j n$-dimensional vector variable.

Definition 6.11. Let $\left\{\Phi_{i}\right\}_{i=1}^{\hat{k}}$ be a family generating $\Phi([0, n T])$ with the affiliated family $\left\{\bar{A}_{i}\right\}_{i=1}^{\hat{k}}$.

(i) A family $\left\{\mathcal{A}_{j}\right\}_{j=1}^{n}$ of symbol matrices is called the symbol family affiliated to $\left\{\Phi_{i}\right\}_{i=1}^{\hat{k}}$ if

$$
\mathcal{A}_{j} \triangleq \mathcal{A}_{j}\left(\left(\alpha_{1}, \alpha_{2}, \ldots, \alpha_{j}\right)\right)=\left(\bar{A}_{1} \alpha_{1}, \ldots, \bar{A}_{\hat{k}} \alpha_{1}, \ldots, \bar{A}_{1} \alpha_{j}, \ldots, \bar{A}_{\hat{k}} \alpha_{j}\right), 1 \leq j \leq n,
$$

where $\left(\alpha_{1}, \alpha_{2}, \ldots, \alpha_{j}\right)$ is given by (6.28).

(ii) When $\left\{\mathcal{A}_{j}\right\}_{j=1}^{n}$ is the symbol family affiliated to $\left\{\Phi_{i}\right\}_{i=1}^{\hat{k}}, \mathcal{A}_{j}$ is called the $j$ th symbol matrix affiliated to $\left\{\Phi_{i}\right\}_{i=1}^{\hat{k}}$.

Clearly, each $\mathcal{A}_{j}$ is a $\left(n-k_{3}\right) \times(n \times \hat{k})$ symbol matrix w.r.t. the vector variable $\left(\alpha_{1}, \alpha_{2}, \ldots, \alpha_{j}\right)$.

Definition 6.12. Let $\left\{\Phi_{i}\right\}_{i=1}^{\hat{k}}$ be a family generating $\Phi([0, n T])$, with the affiliated symbol family $\left\{\mathcal{A}_{j}\right\}_{j=1}^{n}$. The symbol subfamily affiliated to $\left\{\Phi_{i}\right\}_{i=1}^{\hat{k}}$ is defined by

$$
\mathcal{G} \triangleq\left\{\mathcal{A}_{j} \mid \operatorname{Rank}\left(\mathcal{A}_{j}\right)=n-k_{3}, 1 \leq j \leq n\right\} .
$$

Write

$$
D(\mathcal{G})=\min \left\{1 \leq j \leq n \mid \mathcal{A}_{j} \in \mathcal{G}\right\}
$$

Remark 6.13. We must point out that $\mathcal{G} \neq \emptyset$ and hence, $D(\mathcal{G})$ is well defined. In fact, let $\left\{\Phi_{i}\right\}_{i=1}^{\hat{k}}$ be a family generating $\Phi([0, n T])$ (see Def. 6.5). Let $\left\{\bar{A}_{i}\right\}_{i=1}^{\hat{k}}$ and $\mathcal{A}_{n}$ be accordingly the affiliated family and the $n$th symbol matrix affiliated to $\left\{\Phi_{i}\right\}_{i=1}^{\hat{k}}$ (see Defs. 6.6 and 6.11, respectively). We claim that $\mathcal{A}_{n} \in \mathcal{G}$. By Proposition 6.3, 
we have that $I_{n} \in \mathcal{C B}_{A(\cdot)}$. Then, by (6.25) with $B=I_{n}$, we see that $\sum_{i=1}^{\hat{k}} \mathcal{R}\left(\bar{A}_{i}\right)=\mathbb{R}^{n-k_{3}}$. Meanwhile, from (6.29) where $j=n$ and each $\alpha_{i}, i=1, \ldots, n$, takes the value $e_{i}$, it follows that

$$
\mathcal{R}\left(\mathcal{A}_{n}\left(e_{1}, e_{2}, \ldots, e_{n}\right)\right)=\sum_{i=1}^{\hat{k}} \mathcal{R}\left(\bar{A}_{i}\right) .
$$

Hence, $\operatorname{Rank}\left(\mathcal{A}_{n}\left(e_{1}, e_{2}, \ldots, e_{n}\right)\right)=n-k_{3}$. Since $\mathcal{A}_{n}\left(\alpha_{1}, \alpha_{2}, \ldots, \alpha_{n}\right)$ is a $\left(n-k_{3}\right) \times(n \times \hat{k})$ symbol matrix, we see from Definition 6.10 that $\operatorname{Rank}\left(\mathcal{A}_{n}\right)=n-k_{3}$. Then, by $(6.30), \mathcal{A}_{n} \in \mathcal{G}$.

The main result of this section is the following theorem.

Theorem 6.14. Let $\left\{\Phi_{i}\right\}_{i=1}^{\hat{k}}$ be a family generating $\Phi([0, n T])$, with $\left\{\bar{A}_{i}\right\}_{i=1}^{\hat{k}}, \quad\left\{\mathcal{A}_{j}\right\}_{j=1}^{n}$ and $\mathcal{G}$ defined by (6.21), (6.29) and (6.30) respectively. Then, $M\left(\mathcal{C B}_{A(\cdot)}\right)=D(\mathcal{G})$. Moreover, when $\bar{j}=D(\mathcal{G})$, any column vectors $\beta_{1}, \ldots, \beta_{\bar{j}} \in \mathbb{R}^{n}$, with

$$
\operatorname{Rank}\left(\bar{A}_{1} \beta_{1}, \ldots, \bar{A}_{\hat{k}} \beta_{1}, \ldots, \bar{A}_{1} \beta_{\bar{j}}, \ldots, \bar{A}_{\hat{k}} \beta_{\bar{j}}\right)=n-k_{3},
$$

verifies

$$
B \triangleq\left(\beta_{1}, \ldots, \beta_{\bar{j}}\right) \in \mathcal{C B}_{A(\cdot)}
$$

Proof. Let $\bar{j}=D(\mathcal{G})$. By Definition 6.10 , there are vectors $\beta_{1}, \ldots, \beta_{\bar{j}}$ in $\mathbb{R}^{n}$ such that

$$
\operatorname{Rank}\left(\mathcal{A}_{\bar{j}}\right)=\operatorname{Rank}\left(\left(\bar{A}_{1} \beta_{1}, \ldots, \bar{A}_{\hat{k}} \beta_{1}, \bar{A}_{1} \beta_{2}, \ldots, \bar{A}_{\hat{k}} \beta_{2}, \ldots, \bar{A}_{1} \beta_{\bar{j}}, \ldots, \bar{A}_{\hat{k}} \beta_{\bar{j}}\right)\right)=n-k_{3} .
$$

Write $B=\left(\beta_{1}, \ldots, \beta_{\bar{j}}\right)$. Then,

$$
\mathbb{R}^{n-k_{3}}=\mathcal{R}\left(\mathcal{A}_{\bar{j}}\right)=\sum_{i=1}^{\hat{k}} \bar{A}_{i} \mathcal{R}(B) .
$$

By Proposition 6.8 , this yields that $B \in \mathcal{C B}_{A(\cdot)}$. Hence,

$$
D(\mathcal{G})=\bar{j} \geq M\left(\mathcal{C B}_{A(\cdot)}\right) .
$$

Conversely, write $j_{0} \triangleq M\left(\mathcal{C B}_{A(\cdot)}\right)$. Let $\hat{B} \in \mathcal{C B}_{A(\cdot)}$ with $j_{0}$ columns. Write $\hat{B}=\left(b_{1}, \ldots, b_{j_{0}}\right)$, where $b_{i}$ is the $i$ th column of $\hat{B}$. Then, by making use of Proposition 6.8 again, we find

$$
\operatorname{Span}\left\{\bar{A}_{1} b_{1}, \ldots, \bar{A}_{\hat{k}} b_{1}, A_{1} b_{2}, \ldots, \bar{A}_{\hat{k}} b_{2}, \ldots, \bar{A}_{1} b_{j_{0}}, \ldots, \bar{A}_{\hat{k}} b_{j_{0}}\right\}=\mathbb{R}^{n-k_{3}} .
$$

This, along with Definition 6.10 and (6.29), yields

$$
\operatorname{Rank}_{j_{0}}\left(\left(\alpha_{1}, \ldots, \alpha_{j_{0}}\right)\right)=n-k_{3} .
$$

From this, (6.30) and (6.31), we see that

$$
M\left(\mathcal{C B}_{A(\cdot)}\right) \triangleq j_{0} \geq D\left(\mathcal{G}_{A(\cdot)}\right)
$$

This completes the proof. 


\subsection{The special case where $A(\cdot)$ is time-invariant}

Throughout this subsection, we arbitrarily fix a matrix $A \in \mathbb{R}^{n \times n}$. We will apply Theorem 6.14 to this special case to obtain more precise information on the number $M\left(\mathcal{C B}_{A}\right)$. Write

$$
\sigma(A)=\left\{\lambda_{1}, \ldots, \lambda_{r}, \mu_{1}, \bar{\mu}_{1}, \ldots, \mu_{s}, \bar{\mu}_{s}\right\}
$$

Here, $\lambda_{1}, \ldots, \lambda_{r}$ are distinct real eigenvalues of $A$ and $\mu_{1}, \bar{\mu}_{1}, \ldots, \mu_{s}, \bar{\mu}_{s}$ are distinct non-real eigenvalues of $A$. Let $m_{i}, i=1, \ldots, r$, and $\hat{m}_{j}, j=1, \ldots, s$ be accordingly the geometric multiplicities of $\lambda_{i}$ and $\mu_{j}$, i.e., $m_{i}=\operatorname{dim} \operatorname{Ker}\left(A-\lambda_{i} I\right)$ and $\hat{m}_{j}=\operatorname{dim} \operatorname{Ker}\left(A_{c}-\mu_{j}\right)$ with $A_{c}$ the complexification of $A$. Then, $m_{i}$ is the number of the Jordan blocks associated to $\lambda_{i}$, and $\hat{m}_{j}$ is the number of the real Jordan blocks associated to $\left(\mu_{j}, \bar{\mu}_{j}\right)$. The main result in this subsection is as follows:

Theorem 6.15. Let $A \in \mathbb{R}^{n \times n}$. Then

$$
M\left(\mathcal{C B}_{A}\right)=\max _{\lambda \in \sigma(A) \backslash \mathbb{C}^{-}} m(\lambda)
$$

where $m(\lambda)$ is the geometric multiplicity of the eigenvalue $\lambda$.

Before proving this theorem, we introduce some preliminaries. First of all, by the classical matrix theory (see, for instance, Thm. 1 on p. 67 in [10]), there is a non-singular matrix $P \in \mathbb{R}^{n \times n}$ such that

$$
\begin{aligned}
& P^{-1} A P \triangleq \Lambda \\
= & \operatorname{diag}\left\{J_{1}^{\lambda_{1}}, \ldots, J_{m_{1}}^{\lambda_{1}}, \ldots, J_{1}^{\lambda_{r}}, \ldots, J_{m_{r}}^{\lambda_{r}}, J_{1}^{\mu_{1}}, \ldots, J_{\hat{m}_{1}}^{\mu_{1}}, \ldots, J_{1}^{\mu_{s}}, \ldots, J_{\hat{m}_{s}}^{\mu_{s}}\right\},
\end{aligned}
$$

where $J_{l}^{\lambda_{i}}=\left(\begin{array}{cccc}\lambda_{i} & 1 & & \\ & \ddots & \ddots & \\ & & \ddots & 1 \\ & & & \lambda_{i}\end{array}\right), J_{l}^{\mu_{i}}=\left(\begin{array}{cccc}C_{i} & I_{2} & \\ & \ddots & \ddots & \\ & & \ddots & I_{2} \\ & & C_{i}\end{array}\right)$. Here and in what follows, $C_{i}=\left(\begin{array}{c}\operatorname{Re}\left(\mu_{i}\right) \\ -\operatorname{Im}\left(\mu_{i}\right) \\ \operatorname{Re}\left(\mu_{i}\right)\end{array}\right)$, $i=1, \ldots, \bar{m}$. Write

$$
n_{i, l} \triangleq \operatorname{dim} J_{l}^{\lambda_{i}}, \quad 1 \leq i \leq r, 1 \leq l \leq m_{i} ; 2 \hat{n}_{i, l}=\operatorname{dim} J_{l}^{\mu_{i}}, 1 \leq i \leq s, 1 \leq l \leq \hat{m}_{i} .
$$

Let

$$
\hat{e}(k) \triangleq(0, \ldots, 0,1)^{*} \in \mathbb{R}^{k} \text { for each } k \in \mathbb{N} \text {. }
$$

Lemma 6.16. Let $\hat{J}=\left(\begin{array}{cccc}C & I_{2} & & \\ \ddots & \ddots & \\ & \ddots & I_{2} \\ & & C\end{array}\right)$ be a Jordan block with $2 k$ order, where $C=\left(\begin{array}{cc}a & b \\ -b & a\end{array}\right)$, with $a, b \in \mathbb{R}$, $b \neq 0$. Let $\hat{e}(2 k)$ be defined by (6.37). Then

$$
\operatorname{Span}\left\{\hat{e}(2 k), \hat{J} \hat{e}(2 k), \ldots, \hat{J}^{2 k-1} \hat{e}(2 k)\right\}=\mathbb{R}^{2 k} \text {. }
$$

Proof. Write $\left\{e_{1}, \ldots, e_{2 k}\right\}$ for the standard basis of $\mathbb{R}^{2 k}$. Define a $2 k \times 2 k$ orthogonal matrix $E$ by

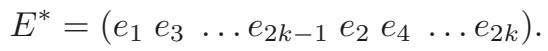


Then, one can directly check what follows:

$$
\widetilde{J} \triangleq E^{*} \hat{J} E=\left(\begin{array}{cc}
A_{k} & b I_{k} \\
-b I_{k} & A_{k}
\end{array}\right) \text {, with } A_{k}=\left(\begin{array}{cccc}
a & 1 & & \\
& \ddots & \ddots & \\
& & a & 1 \\
& & & a
\end{array}\right) \text { a } k \times k \text { matrix }
$$

and

$$
\hat{e}(2 k)=E \hat{e}(2 k) \text {. }
$$

Let $\hat{I}_{2 k}=\left(\begin{array}{cc}\frac{1}{2} I_{k} & \frac{1}{2} I_{k} \\ \frac{i}{2} I_{k} & -\frac{i}{2} I_{k}\end{array}\right)$. Then,

$$
\widetilde{J}=\hat{I}_{2 k}\left(\begin{array}{ll}
J_{k} & \\
& \bar{J}_{k}
\end{array}\right)\left(\hat{I}_{2 k}\right)^{-1}, \quad \text { with } J_{k}=\left(\begin{array}{cccc}
a+i b & 1 & & \\
& \ddots & \ddots & \\
& & \ddots & 1 \\
& & a+i b
\end{array}\right) \text { a } k \times k \text { matrix. }
$$

Thus, one can directly check that

$$
\left|\hat{e}(2 k), \hat{J} \hat{e}(2 k), \ldots, \hat{J}^{2 k-1} \hat{e}(2 k)\right|=|E|\left|\hat{I}_{2 k}\right|\left|\left(\begin{array}{c}
\hat{e}(k) \\
\hat{e}(k)
\end{array}\right),\left(\begin{array}{c}
J_{k} \hat{e}(k) \\
\bar{J}_{k} \hat{e}(k)
\end{array}\right), \ldots,\left(\begin{array}{c}
J_{k}^{2 k-1} \hat{e}(k) \\
\bar{J}_{k}^{2 k-1} \hat{e}(k)
\end{array}\right)\right|,
$$

where $|S|$ denotes to the determinant of a square matrix $S$. From this, (6.38) will follow from non-singularity of the matrix:

$$
H=\left(\left(\begin{array}{c}
\hat{e}(k) \\
\hat{e}(k)
\end{array}\right),\left(\begin{array}{c}
J_{k} \hat{e}(k) \\
\bar{J}_{k} \hat{e}(k)
\end{array}\right), \ldots,\left(\begin{array}{c}
J_{k}^{2 k-1} \hat{e}(k) \\
\bar{J}_{k}^{2 k-1} \hat{e}(k)
\end{array}\right)\right) .
$$

To prove that $H$ is invertible, we let $\left(a_{0}, a_{1}, \ldots, a_{2 k-1}\right)^{*} \in \mathbb{C}^{2 k}$ verify

$$
H\left(a_{0}, a_{1}, \ldots, a_{2 k-1}\right)^{*}=0_{2 k \times 1} .
$$

Define a polynomial

$$
f(\lambda)=a_{2 k-1} \lambda^{2 k-1}+a_{2 k-2} \lambda^{2 k-2}+\ldots+a_{1} \lambda+a_{0} .
$$

From (6.39),

$$
f\left(J_{k}\right) \hat{e}(k)=f\left(\bar{J}_{k}\right) \hat{e}(k)=0 .
$$

Hence, there are polynomials $g_{1}, g_{2}, h_{1}, h_{2}$ (with complex coefficients) such that

$$
f(\lambda)=g_{1}(\lambda)(\lambda-a-i b)^{k}+h_{1}(\lambda), \quad f(\lambda)=g_{2}(\lambda)(\lambda-a+i b)^{k}+h_{2}(\lambda),
$$

where $\partial\left(h_{1}\right)<k, \partial\left(h_{2}\right)<k$ (Here, $\partial\left(h_{i}\right)$ denotes the degree of $\left.h_{i}\right)$. Because

$$
\left(J_{k}-(a+i b) I_{k}\right)^{k}=0=\left(\bar{J}_{k}-(a-i b) I_{k}\right)^{k},
$$

it follows from (6.40) that

$$
h_{1}\left(J_{k}\right) \hat{e}(k)=0 \text { and } h_{2}\left(\bar{J}_{k}\right) \hat{e}(k)=0 .
$$

This, along with the invertibility of $\left(\hat{e}(k), J_{k} \hat{e}(k), \ldots, J_{k}^{k-1} \hat{e}(k)\right)$ and $\left(\hat{e}(k), \bar{J}_{k} \hat{e}(k), \ldots, \bar{J}_{k}^{k-1} \hat{e}(k)\right)$, implies that

$$
h_{1}(\lambda) \equiv 0 \text { and } h_{2}(\lambda) \equiv 0 .
$$

Thus, $f$ is a multiple of $(\lambda-a-i b)^{k}(\lambda-a-i b)^{k}$. This, along with $\partial(f)<2 k$, indicates $f(\lambda) \equiv 0$. Therefore, $\left(a_{0}, a_{1}, \ldots, a_{2 k-1}\right)^{*}=0$ and matrix $H$ is non-singular. 
Proposition 6.17. Let $A_{1} \in \mathbb{R}^{p \times p}, A_{2} \in \mathbb{R}^{q \times q}, B_{1} \in \mathbb{R}^{p \times r}, B_{2} \in \mathbb{R}^{q \times r}$. Suppose that controlled systems

$$
\dot{x}(t)=A_{1} x(t)+B_{1} u(t)
$$

and

$$
\dot{y}(t)=A_{2} y(t)+B_{2} u(t)
$$

are controllable respectively. If

$$
\sigma\left(A_{1}\right) \bigcap \sigma\left(A_{2}\right)=\varnothing
$$

then the system

$$
\frac{\mathrm{d}}{\mathrm{d} t}\left(\begin{array}{l}
x \\
y
\end{array}\right)(t)=\left(\begin{array}{ll}
A_{1} & \\
& A_{2}
\end{array}\right)\left(\begin{array}{l}
x \\
y
\end{array}\right)(t)+\left(\begin{array}{l}
B_{1} \\
B_{2}
\end{array}\right) u(t)
$$

is also controllable.

Proof. Let $\left(x_{0}, y_{0}\right) \in \mathbb{R}^{p} \times \mathbb{R}^{q}$ verify

$$
\left(B_{1}^{*}, B_{2}^{*}\right)\left(\begin{array}{ll}
\mathrm{e}^{A_{1}^{*}} & \\
& \mathrm{e}^{A_{2}^{*}}
\end{array}\right)\left(\begin{array}{l}
x_{0} \\
y_{0}
\end{array}\right)=0 .
$$

We aim to show that $\left(x_{0}, y_{0}\right)=0$. When this is done, the controllability of (6.42) follows from the classical O.D.E control theory (see, for instance, Thm. 1.7 on p. 112 in [16]). Clearly, (6.43) is equivalent to

$$
B_{1}^{*} \mathrm{e}^{A_{1}^{*} t} x_{0}+B_{2}^{*} \mathrm{e}^{A_{2}^{*} t} y_{0}=0 \text { for all } t \geq 0 .
$$

By differentiating (6.44) times, we obtain

$$
B_{1}^{*} \mathrm{e}^{A_{1}^{*} t} A_{1}^{k} x_{0}+B_{2}^{*} \mathrm{e}^{A_{2}^{*} t} A_{2}^{k} y_{0}=0 \quad k=1,2, \ldots
$$

Let $f_{1}(\cdot), f_{2}(\cdot)$ be accordingly the characteristic polynomials of $A_{1}$ and $A_{2}$. Because of $(6.41), f_{1}(\cdot), f_{2}(\cdot)$ are coprime. Thus there are polynomials $g_{1}(\cdot)$ and $g_{2}(\cdot)$ such that

$$
g_{1}(\cdot) f_{1}(\cdot)+g_{2}(\cdot) f_{2}(\cdot) \equiv 1 .
$$

It follows from (6.45) that

$$
B_{1}^{*} \mathrm{e}^{A_{1}^{*} t}\left(g_{1} \times f_{1}\right)\left(A_{1}\right) x_{0}+B_{2}^{*} \mathrm{e}^{A_{2}^{*} t}\left(g_{1} \times f_{1}\right)\left(A_{2}\right) y_{0}=0 .
$$

On the other hand, by the Hamilton-Cayley Theorem,

$$
f_{1}\left(A_{1}\right)=0, \quad f_{2}\left(A_{2}\right)=0 .
$$

Hence, from (6.46), (6.48), (6.47) and (6.48)

$$
\begin{aligned}
& B_{2}^{*} \mathrm{e}^{A_{2}^{*} t} y_{0}=B_{2}^{*} \mathrm{e}^{A_{2}^{*} t} I y_{0}=B_{2}^{*} \mathrm{e}^{A_{2}^{*} t}\left(g_{1} \times f_{1}+g_{2} \times f_{2}\right)\left(A_{2}\right) y_{0} \\
= & B_{2}^{*} \mathrm{e}^{A_{2}^{*} t}\left(g_{1} \times f_{1}\right)\left(A_{2}\right) y_{0}=-B_{1}^{*} \mathrm{e}^{A_{1}^{*} t}\left(g_{1} \times f_{1}\right)\left(A_{1}\right) x_{0}=0 .
\end{aligned}
$$

Since $\left[A_{2}, B_{2}\right]$ is controllable, the above implies $y_{0}=0$. Likewise, $x_{0}=0$. This completes the proof. 
Since the the Kalman rank condition for $[A, B]$ is equivalent to the controllability of $[A, B]$, we have following consequence of Proposition 6.17:

Corollary 6.18. When

$$
\operatorname{Rank}\left(B_{1}, A_{1} B_{1}, \ldots, A_{1}^{p-1} B_{1}\right)=p, \quad \operatorname{Rank}\left(B_{2}, A_{2} B_{2}, \ldots, A_{2}^{q-1} B_{2}\right)=q,
$$

and (6.41) stands,

$$
\operatorname{Rank}\left(\left(\begin{array}{l}
B_{1} \\
B_{2}
\end{array}\right),\left(\begin{array}{ll}
A_{1} & \\
& A_{2}
\end{array}\right)\left(\begin{array}{l}
B_{1} \\
B_{2}
\end{array}\right), \ldots,\left(\begin{array}{ll}
A_{1} & \\
& A_{2}
\end{array}\right)^{p+q-1}\left(\begin{array}{l}
B_{1} \\
B_{2}
\end{array}\right)\right)=p+q .
$$

Proof of Theorem 6.15. First we prove (6.34) for the case where $\sigma(A) \cap \mathbb{C}^{-}=\varnothing$. In this case, $k_{3}=0$ and $Q=I$, where $k_{3}$ and $Q$ are given by (6.5) and (6.4) respectively. Moreover, $\bar{k}$ (defined by (6.12)) is the degree of the minimal polynomial for matrix $A$. (This will not be used in our proof.) By the Hamilton-Cayley Theorem,

$$
\operatorname{Span}\left\{\mathrm{e}^{-A t}, t \in[0, n T]\right\}=\operatorname{Span}\left\{I, A, A^{2}, \ldots, A^{n-1}\right\} .
$$

Hence, $\left\{I, A, \ldots, A^{n-1}\right\}$ is a family generating $\Phi([0, n T])$ with $\Phi(t)=\mathrm{e}^{-t A}$ (see Def. 6.5). By Definitions 6.6, 6.11, the family and the symbol family affiliated to $\left\{I, A, A^{2}, \ldots, A^{n-1}\right\}$ are accordingly $\left\{A^{i-1}\right\}_{i=1}^{n}$ and $\left\{\mathcal{A}_{j}\right\}_{j=1}^{n}$ with

$$
\mathcal{A}_{j} \triangleq \mathcal{A}_{j}\left(\left(\alpha_{1}, \ldots, a_{j}\right)\right)=\left(\alpha_{1}, \ldots, A^{n-1} \alpha_{1}, \ldots, \alpha_{j}, \ldots, A^{n-1} \alpha_{j}\right), \quad 1 \leq j \leq n .
$$

Let

$$
\hat{\mathcal{A}}_{j} \triangleq \hat{\mathcal{A}}_{j}\left(\left(\alpha_{1}, \ldots, \alpha_{j}\right)\right) \triangleq\left(\alpha_{1}, \ldots, \Lambda^{n-1} \alpha_{1}, \alpha_{j}, \ldots, \Lambda^{n-1} \alpha_{j}\right), 1 \leq j \leq n,
$$

where $\Lambda$ is given by (6.35). Clearly, $\hat{\mathcal{A}}_{j}$ is a $n \times n j$ symbol matrix w.r.t. $\left(\alpha_{1}, \ldots, \alpha_{j}\right)$, and

$$
\left.\operatorname{Rank}\left(\mathcal{A}_{j}\left(\left(a_{1}, \ldots, a_{j}\right)\right)\right)=\operatorname{Rank}\left(\hat{\mathcal{A}}_{j}\left(\alpha_{1}, \ldots, a_{j}\right)\right)\right) .
$$

Write

$$
\bar{m}=\max \left\{m_{1}, \ldots, m_{r}, \hat{m}_{1}, \ldots, \hat{m}_{s}\right\} .
$$

By Theorem 6.14 and Definition 6.12 (where $k_{3}=0$ ), the statement (6.34) is equivalent to the following:

$$
\operatorname{Rank}\left(\hat{\mathcal{A}}_{j}\left(\left(\alpha_{1}, \ldots, \alpha_{j}\right)\right)\right)<n \text {, when } j<\bar{m},
$$

and

$$
\operatorname{Rank}\left(\hat{\mathcal{A}}_{\bar{m}}\left(\left(\alpha_{1}, \ldots, \alpha_{\bar{m}}\right)\right)\right)=n \text {. }
$$

Step 1. The proof of (6.54)

Suppose that $j<\bar{m}$. By Definition 6.10 and (6.52), to prove (6.54), it suffices to show that each $n$-order sub-determinant of $\hat{\mathcal{A}}_{j}\left(\left(\alpha_{1}, \ldots, a_{j}\right)\right)$ is 0 . Seeking a contradiction, we suppose that it did not stand. Then, there would be vectors $\bar{\alpha}_{1}, \bar{\alpha}_{2}, \ldots, \bar{\alpha}_{j} \in \mathbb{R}^{n}$ such that one of $n$-order sub-determinant of $\hat{\mathcal{A}}_{j}\left(\left(\hat{\alpha}_{1}, \hat{\alpha}_{2}, \ldots, \hat{\alpha}_{j}\right)\right)$ is not zero. Since the matrix $\hat{\mathcal{A}}_{j}\left(\left(\bar{\alpha}_{1}, \bar{\alpha}_{2}, \ldots, \bar{\alpha}_{j}\right)\right)$ has exactly $n$ rows, any group of distinct row vectors in $\hat{\mathcal{A}}_{j}\left(\left(\bar{\alpha}_{1}, \bar{\alpha}_{2}, \ldots, \bar{\alpha}_{j}\right)\right)$ is linearly independent.

Without loss of generality, we can assume that either $\bar{m}=m_{1}$ or $\bar{m}=\hat{m}_{1}$. We will deduce a contradiction for each case.

The first case where $\bar{m}=m_{1}$ : write

$$
n_{l} \triangleq \sum_{i=1}^{l} n_{1, i}, \quad l=1,2, \ldots, \bar{m}
$$


where $n_{1, i}$ is defined by (6.36). Let

$$
\hat{\alpha}_{i} \triangleq\left(e_{n_{1}}, \ldots, e_{n_{\bar{m}}}\right)^{*} \bar{\alpha}_{i} \in \mathbb{R}^{\bar{m}}, \quad i=1, \ldots, j,
$$

where and throughout the proof, $\left\{e_{1}, \ldots, e_{n}\right\}$ stands for the standard basis of $\mathbb{R}^{n}$ with each $e_{i}$ a column vector. Notice that if we write $\bar{\alpha}_{i}=\left(x_{1}^{i}, x_{2}^{i}, \ldots, x_{n}^{i}\right)^{*}$, with $x_{l}^{i} \in \mathbb{R}$, then $\hat{\alpha}_{i}=\left(x_{n_{1}}^{i}, x_{n_{2}}^{i}, \ldots, x_{n_{\bar{m}}}^{i}\right)^{*}$. From $(6.35)$ and (6.57), one has that when $1 \leq i \leq j$ and $k \in \mathbb{N}$,

$$
\left(e_{n_{1}}, e_{n_{2}}, \ldots, e_{n_{\bar{m}}}\right)^{*} \Lambda^{k} \bar{\alpha}_{i}=\left(\begin{array}{c}
e_{n_{1}}^{*} \\
e_{n_{2}}^{*} \\
\ldots \\
e_{n_{\bar{m}}}^{*}
\end{array}\right) \Lambda^{k} \bar{\alpha}_{i}=\lambda_{1}^{k}\left(\begin{array}{c}
e_{n_{1}}^{*} \\
e_{n_{2}}^{*} \\
\ldots \\
e_{n_{\bar{m}}}^{*}
\end{array}\right) \bar{\alpha}_{i}=\lambda_{1}^{k} \hat{\alpha}_{i} .
$$

This, along with (6.51), indicates

$$
\begin{aligned}
& \left(e_{n_{1}}, e_{n_{2}}, \ldots, e_{n_{\bar{m}}}\right)^{*} \hat{\mathcal{A}}_{j}\left(\bar{\alpha}_{1}, \bar{\alpha}_{2}, \ldots, \bar{\alpha}_{j}\right) \\
= & \left(e_{n_{1}}, e_{n_{2}}, \ldots, e_{n_{\bar{n}}}\right)^{*}\left(\bar{\alpha}_{1}, \ldots, \Lambda^{n-1} \bar{\alpha}_{1}, \bar{\alpha}_{2}, \ldots, \Lambda^{n-1} \bar{\alpha}_{2}, \ldots, \bar{\alpha}_{j}, \ldots, \Lambda^{n-1} \bar{\alpha}_{j}\right) \\
= & \left(\hat{\alpha}_{1}, \lambda_{1} \hat{\alpha}_{1}, \ldots, \lambda_{1}^{n-1} \hat{\alpha}_{1}, \hat{\alpha}_{2}, \lambda_{1} \hat{\alpha}_{2}, \ldots, \lambda_{1}^{n-1} \hat{\alpha}_{2}, \ldots, \hat{\alpha}_{j}, \lambda_{1} \hat{\alpha}_{j}, \ldots, \lambda_{1}^{n-1} \hat{\alpha}_{j}\right) .
\end{aligned}
$$

Clearly, any maximal independent group of column vectors in the matrix on the right side of (6.58) has at most $j$ vectors. Thus, the rank of the matrix on the right side of $(6.58)$ is less than or equals to $j(<\bar{m})$.

On the other hand, the matrix on the left side of (6.58) consists of $\bar{m}$ row vectors which are exactly $n_{1}$ th, $n_{2}$ th, $\ldots$ and $n_{\bar{m}}$ th rows in $\hat{\mathcal{A}}_{j}\left(\bar{\alpha}_{1}, \bar{\alpha}_{2}, \ldots, \bar{\alpha}_{j}\right)$. Since the rank of the matrix on the right side of (6.58) is less than $\bar{m}$, these row vectors are linearly dependent, which contradicts with the fact that any group of distinct row vectors in $\hat{\mathcal{A}}_{j}\left(\left(\bar{\alpha}_{1}, \bar{\alpha}_{2}, \ldots, \bar{\alpha}_{j}\right)\right)$ is linearly independent.

The second case where $\bar{m}=\hat{m}_{1}$. Let

$$
\tilde{n}=\operatorname{dim}\left(\operatorname{diag}\left\{J_{1}^{\lambda_{1}}, \ldots, J_{m_{1}}^{\lambda_{1}}, \ldots, J_{1}^{\lambda_{r}}, \ldots, J_{m_{r}}^{\lambda_{r}}\right\}\right)
$$

and

$$
\hat{n}_{l}=\tilde{n}+\sum_{i=1}^{l} 2 \hat{n}_{1, i}, \quad l=1,2, \ldots, \bar{m},
$$

where $\hat{n}_{1, i}$ is defined by (6.36). By (6.35), one can easily check that

$$
\left(\begin{array}{c}
e_{\hat{n}_{l}-1}^{*} \\
e_{\hat{n}_{l}}^{*}
\end{array}\right) \Lambda^{k}=C_{1}^{k}\left(\begin{array}{c}
e_{\hat{n}_{l}-1}^{*} \\
e_{\hat{n}_{l}}^{*}
\end{array}\right), l=1, \ldots, \bar{m}, k \in \mathbb{N} .
$$

For each $\bar{\alpha}_{i}$, with $i \in\{1, \ldots, j\}$, we define a vector

$$
\widetilde{\alpha}_{i} \triangleq\left(\begin{array}{lllll}
e_{\hat{n}_{1}-1} & e_{\hat{n}_{1}} & e_{\hat{n}_{2}-1} & e_{\hat{n}_{2}} \ldots e_{\hat{n}_{\bar{m}}-1} & e_{\hat{n}_{\bar{m}}}
\end{array}\right)^{*} \bar{\alpha}_{i} \in \mathbb{R}^{2 \bar{m}} .
$$

Notice that, if we denote $\bar{\alpha}_{i}=\left(x_{1}^{i}, \ldots, x_{n}^{i}\right)^{*}$, with each $x_{k}^{i} \in \mathbb{R}$, then

$$
\widetilde{\alpha}_{i}=\left(x_{\hat{n}_{1}-1}^{i}, x_{\hat{n}_{1}}^{i}, \ldots, x_{\hat{n}_{\bar{m}}-1}^{i}, x_{\hat{n}_{\bar{m}}}^{i}\right) \triangleq\left(\widetilde{\alpha}_{i 1}^{*}, \widetilde{\alpha}_{i 2}^{*}, \ldots, \widetilde{\alpha}_{i \bar{m}}^{*}\right), i=1, \ldots, j,
$$

where $\widetilde{\alpha}_{i k}^{*}=\left(x_{\hat{n}_{k}-1}^{i}, x_{\hat{n}_{k}}^{i}\right)^{*}, \quad k=1, \ldots, \bar{m}$. By (6.35), (6.51), (6.61) and (6.62), one can directly verify

$$
\begin{aligned}
& \left(\begin{array}{lllll}
e_{\hat{n}_{1}-1} & e_{\hat{n}_{1}} & e_{\hat{n}_{2}-1} & e_{\hat{n}_{2}}, \ldots, e_{\hat{n}_{\bar{m}}-1} & e_{\hat{n}_{\bar{m}}}
\end{array}\right)^{*} \hat{\mathcal{A}}_{j}\left(\bar{\alpha}_{1}, \bar{\alpha}_{2}, \ldots, \bar{\alpha}_{j}\right) \\
& =\left(\begin{array}{cccccccccc}
\widetilde{\alpha}_{11} & C_{1} \widetilde{\alpha}_{11} & \ldots & C_{1}^{n-1} \widetilde{\alpha}_{11} & \ldots & \widetilde{\alpha}_{j 1} & C_{1} \widetilde{\alpha}_{j 1} & \ldots & C_{1}^{n-1} \widetilde{\alpha}_{j 1} \\
\widetilde{\alpha}_{12} & C_{1} \widetilde{\alpha}_{12} & \ldots & C_{1}^{n-1} \widetilde{\alpha}_{12} & \ldots & \widetilde{\alpha}_{j 2} & C_{1} \widetilde{\alpha}_{j 2} & \ldots & C_{1}^{n-1} \widetilde{\alpha}_{j 2} \\
\ldots & \ldots & \ldots & \ldots & \ldots & \ldots & \ldots & \ldots & \ldots \\
\widetilde{\alpha}_{1 \bar{m}} & C_{1} \widetilde{\alpha}_{1 \bar{m}} & \ldots & C_{1}^{n-1} \widetilde{\alpha}_{1 \bar{m}} & \ldots & \widetilde{\alpha}_{j \bar{m}} & C_{1} \widetilde{\alpha}_{j \bar{m}} & \ldots & C_{1}^{n-1} \widetilde{\alpha}_{j \bar{m}}
\end{array}\right),
\end{aligned}
$$


Because $C_{1}$ is a $2 \times 2$ matrix, by the Hamilton Cayley Theorem, each $C_{1}^{k}$, with $k \in \mathbb{N}$, is a linear combination of $I_{2}$ and $C_{1}$. Thus, any maximal independent group of column vectors in the matrix on the right side of (6.63) has at most $2 j$ vectors. Therefore, the rank of the matrix on the right side of (6.63) is less than or equals to $2 j(<2 \bar{m})$. On the other hand, the matrix on the left side of (6.63) consists of $2 \bar{m}$ row vectors which are exactly $\left(\hat{n}_{1}-1\right)$ th, $\hat{n}_{1}$ th $, \ldots,\left(\hat{n}_{\bar{m}}-1\right)$ th and $\hat{n}_{\bar{m}}$ th rows of $\hat{\mathcal{A}}_{j}\left(\bar{\alpha}_{1}, \bar{\alpha}_{2}, \ldots, \bar{\alpha}_{j}\right)$. Since the rank of the matrix on the right side of (6.63) is less than $2 \bar{m})$, these $2 \bar{m}$ row vectors are linearly dependent, which contradicts to the fact that any group of distinct row vectors in $\hat{\mathcal{A}}_{j}\left(\left(\bar{\alpha}_{1}, \bar{\alpha}_{2}, \ldots, \bar{\alpha}_{j}\right)\right)$ is linearly independent.

In summary, we conclude that (6.54) stands.

Step 2. The proof of $(6.55)$

By Definition 6.10, it suffices to structure vectors $\xi_{1}, \ldots, \xi_{\bar{m}}$ in $\mathbb{R}^{n}$ such that

$$
\operatorname{Rank}\left(\left(\hat{\mathcal{A}}_{\bar{m}}\left(\left(\xi_{1}, \xi_{2}, \ldots, \xi_{\bar{m}}\right)\right)\right)\right)=n .
$$

For this purpose, we first let, for each $k \in \mathbb{N}, \hat{e}(k)$ be given by (6.37). Then, we construct, for each $k \in\{1, \ldots, \bar{m}\}$, a vector $\xi_{k}$ in $\mathbb{R}^{n}$ by setting

$$
\xi_{k}^{*}=\left(\left(f_{1,1}^{k}\right)^{*} \ldots\left(f_{1, m_{1}}^{k}\right)^{*} \ldots\left(f_{r, 1}^{k}\right)^{*} \ldots\left(f_{r, m_{r}}^{*}\right)^{*}\left(g_{1,1}^{k}\right)^{*} \ldots\left(g_{1, \hat{m}_{1}}^{k}\right)^{*} \ldots\left(g_{s, 1}^{k}\right)^{k} \ldots\left(g_{s, \hat{m}_{s}}^{k}\right)^{*}\right),
$$

where $f_{i, l}^{k} \in \mathbb{R}^{n_{i, l}}$, with $1 \leq i \leq r$ and $1 \leq l \leq m_{i}$, is defined in the following manner:

$$
\text { when } l=k, \quad f_{i, l}^{k}=\hat{e}\left(n_{n_{i, l}}\right) ; \quad \text { when } l \neq k, \quad f_{i, l}^{k}=0_{n_{i, l} \times 1},
$$

and $g_{i, l}^{k} \mathbb{R}^{2 \hat{n}_{i, l}}$ with $1 \leq i \leq s$ and $1 \leq l \leq \hat{m}_{i}$, is defined in the following manner:

$$
\text { when } l=k, \quad g_{i, l}^{k}=\hat{e}\left(2 \hat{n}_{n_{i, l}}\right) ; \quad \text { when } l \neq k, \quad g_{i, l}^{k}=0_{2 n_{i, l} \times 1} \text {. }
$$

Two observations (follow accordingly from (6.35) and Lemma 6.16) are in order:

$$
\begin{aligned}
& \operatorname{Span}\left\{\hat{e}\left(n_{i, l}\right), J_{l}^{\lambda_{i}} \hat{e}\left(n_{i, l}\right), \ldots,\left(J_{l}^{\lambda_{i}}\right)^{n_{i, l}-1} \hat{e}\left(n_{i, l}\right)\right\}=\mathbb{R}^{n_{i, l}} \text {, when } 1 \leq i \leq r, 1 \leq l \leq m_{i} ; \\
& \operatorname{Span}\left\{\hat{e}\left(2 \hat{n}_{i, l}\right), J_{l}^{\mu_{i}} \hat{e}\left(2 \hat{n}_{i, l}\right), \ldots,\left(J_{l}^{\mu_{i}}\right)^{2 \hat{n}_{i, l}-1} \hat{e}\left(2 \hat{n}_{i, l}\right)\right\}=\mathbb{R}^{2 \hat{n}_{i, l}} \text {, when } 1 \leq s, 1 \leq l \leq \hat{m}_{i} .
\end{aligned}
$$

Write $\mathcal{N}_{k}$, with $1 \leq k \leq \bar{m}$, for the set of all such $k^{\prime} \in\{1,2, \ldots, n\}$ that the $k^{\prime}$ th row of $\Lambda$ contains one of rows in one of blocks $J_{k}^{\lambda_{1}}, \ldots, J_{k}^{\lambda_{r}}, \hat{J}_{k}^{\mu_{1}}, \ldots, \hat{J}_{k}^{\mu_{s}}$ (where $J_{k}^{\lambda_{i}}=\varnothing$ or $\hat{J}_{k}^{\mu_{j}}=\varnothing$ if $m_{i}<k$ or $\hat{m}_{j}<k$ ). From (6.35), (6.65), (6.66), (6.67) and Corollary 6.18, we obtain

$$
\operatorname{Span}\left\{\xi_{k}, \Lambda \xi_{k}, \ldots, \Lambda^{n-1} \xi_{k}\right\}=\operatorname{Span}\left\{e_{l}, \quad l \in \mathcal{N}_{k}\right\} .
$$

From the definition of $\mathcal{N}_{k}$, One has

$$
\bigcup_{k=1}^{\bar{m}} \mathcal{N}_{k}=\{1,2, \ldots, n\} \text { and } \mathcal{N}_{i} \bigcap \mathcal{N}_{j}=\varnothing \text {, when } i \neq j .
$$

Finally, by the definition of $\hat{\mathcal{A}}_{\bar{m}}\left(\left(\alpha_{1}, \alpha_{2}, \ldots, \alpha_{\bar{m}}\right)\right)$ (see (6.51)), (6.68) and (6.69), we obtain (6.64). Thus we prove the case when $\sigma(A) \subset \mathbb{C}^{-}$.

The remaining is to prove the case $\sigma(A) \cap \mathbb{C}^{-} \neq \varnothing$. In this case, we can apply Lemma 2.7 to decouple the system $x^{\prime}=A x$ into two subsystems: $k_{3}$-order $x_{1}^{\prime}=A_{1} x_{1}$ and $\left(n-k_{3}\right)$-order $x_{2}^{\prime}=A_{2} x_{2}$, with $\sigma\left(A_{1}\right) \subset \mathbb{C}^{-}$and $\sigma\left(A_{2}\right) \cap \mathbb{C}^{-}=\varnothing$. If $\left(n-k_{3}\right) \times k$-order matrix $B_{2} \in \mathcal{C B}_{A_{2}}$, then matrix

$$
\left(\begin{array}{c}
0_{k_{3} \times k} \\
B_{2}
\end{array}\right) \in \mathcal{C B}_{A}
$$


which leads to

$$
M\left(\mathcal{C B}_{A}\right) \leq M\left(\mathcal{C B}_{A_{2}}\right) .
$$

On the other hand, notice $\mathcal{P}=\left(\begin{array}{cc}\mathrm{e}^{A_{1} T} & \\ & \mathrm{e}^{A_{2} T}\end{array}\right)$. Select $Q=\left(\begin{array}{cc}I_{k_{3}} & \\ & I_{n-k_{3}}\end{array}\right)$ in equation (6.4). Let

$$
\Phi_{i}=\left(\begin{array}{cc}
A_{1}^{i-1} & \\
& A_{2}^{i-1}
\end{array}\right), \quad i \in\{1,2, \ldots, n\},
$$

a family generating $\Phi([0, n T])$ in (6.20). Thus the family affiliated to $\left\{\Phi_{i}\right\}_{i=1}^{n}$ is

$$
\bar{A}_{i}=\left(0_{\left(n-k_{3}\right) \times k_{3}}, A_{2}^{i-1}\right), \quad i \in\{1,2, \ldots, n\} .
$$

For any $B \in \mathcal{C B}_{A}$, assume $k$ the column number of $B$. Let

$$
B_{2}=\left(0_{\left(n-k_{3}\right) \times k_{3}}, I_{n-k_{3}}\right) B \in \mathbb{R}^{\left(n-k_{3}\right) \times k} .
$$

By Proposition 6.8, one can directly check that

$$
\begin{aligned}
& B \in \mathcal{C B}_{A} \Leftrightarrow \sum_{i=1}^{n} \bar{A}_{i} \mathcal{R}(B)=\mathbb{R}^{n-k_{3}} \\
\Leftrightarrow & \sum_{i=1}^{n} A_{2}^{i-1}\left(0_{\left(n-k_{3}\right) \times k_{3}}, I_{n-k_{3}}\right) \mathcal{R}(B)=\mathbb{R}^{n-k_{3}} \\
\Leftrightarrow & \sum_{i=1}^{n} A_{2}^{i-1} \mathcal{R}\left(B_{2}\right)=\mathbb{R}^{n-k_{3}} \\
\Leftrightarrow & B_{2} \in \mathcal{C} \mathcal{B}_{A_{2}},
\end{aligned}
$$

which leads to $M\left(\mathcal{C B}_{A_{2}}\right) \leq M\left(\mathcal{C B}_{A}\right)$. That together with (6.70) implies that

$$
M\left(\mathcal{C B}_{A}\right)=M\left(\mathcal{C B}_{A_{2}}\right) .
$$

Based the result of the first case, we derived the desired result (6.34).

Remark 6.19. In the proof above, we also provide a way to structure a matrix $B$ with the column number $M\left(\mathcal{C B}_{A}\right)$. More precisely, $B=P\left(\xi_{1}, \xi_{2}, \ldots, \xi_{\bar{m}}\right)$, where $P$ is given by $(6.35)$ and $\xi_{k}$, with $1 \leq k \leq \bar{m}$, is given by $(6.65)$.

\subsection{The case when both $A(\cdot)$ and $B(\cdot)$ are time-varying}

Let $A(\cdot) \in L^{\infty}\left(\mathbb{R}^{+} ; \mathbb{R}^{n}\right)$ be $T$-periodic. Define

$$
\mathcal{C B}_{A(\cdot)}^{1}=\left\{B(\cdot) \in L^{\infty}\left(\mathbb{R}^{+} ; \mathbb{R}^{n \times m}\right) \mid m \in \mathbb{N},[A(\cdot), B(\cdot)] \text { is T-periodically stabilizable }\right\} .
$$

For each $B(\cdot) \in \mathcal{C B}_{A(\cdot)}^{1}$, denote by $N(B(\cdot))$ the number of columns of $B(\cdot)$. Set

$$
N\left(\mathcal{C B}_{A(\cdot)}^{1}\right) \triangleq \min \left\{N(B(\cdot)) \mid B(\cdot) \in \mathcal{C B}_{A(\cdot)}^{1}\right\} .
$$

The purpose of this subsection is to show, with the aid of Theorem 1.2, the following theorem.

Theorem 6.20. It holds that $M\left(\mathcal{C B}_{A(\cdot)}\right)=1$. Namely, there is a T-periodic function $B(\cdot)$ in $L^{\infty}\left(\mathbb{R}^{+} ; \mathbb{R}^{n \times 1}\right)$ such that $[A(\cdot), B(\cdot)]$ is periodically stabilizable.

To prove the above theorem, we need following result on the Floquet's theory (see $[8,18]$ ). 
Lemma 6.21. Let $A(\cdot)$ be a T-periodic $A(\cdot) n \times n$ real matrix-valued function. Then, there is a T-periodic transform $\mathcal{T}(\cdot) \in C^{1}\left(\mathbb{R} ; \mathbb{R}^{n \times n}\right)$, with non-singular $\mathcal{T}(t)$ for each $t$, such that the time-varying system $\dot{x}(t)=$ $A(t) x(t)$ can be transform into an autonomous system with respect to $z$ by the transformation $x=\mathcal{T}(t) z$.

The above transformation $\mathcal{T}(\cdot)$ is called the Lyapunov transform (associated with $A(\cdot)$ ).

Proof of Theorem 6.20. Let $\mathcal{T}(\cdot)$ be the Lyapunov transform given by Lemma 6.21. Then,

$$
\mathcal{T}^{-1}(t)(A(t) \mathcal{T}(t)-\dot{\mathcal{T}}(t)) \equiv \hat{A}, \quad t \geq 0
$$

for some $\hat{A} \in \mathbb{R}^{n \times n}$. Let $\left\{e_{1}, \ldots, e_{n}\right\}$ be the standard basis of $\mathbb{R}^{n}$. Write

$$
b_{k}=\mathrm{e}^{(k T / n) \hat{A}} e_{k+1}, \quad k=0, \ldots, n-1 .
$$

Define $\hat{B}(\cdot)$ by

$$
\hat{B}(t)=\sum_{k=0}^{n-1} \chi_{[k T / n,(k+1) T / n)} b_{k} \text { for } t \in[0, T) ; \hat{B}(t)=\hat{B}(t-T) \text { for } t \geq T .
$$

Write $B(\cdot)=\mathcal{T}(\cdot) \hat{B}(\cdot)$. Clearly, $B(\cdot)$ is a $T$-periodic function in $L^{\infty}\left(\mathbb{R}^{+} ; \mathbb{R}^{n \times 1}\right)$. According to Lemma 6.21 and equation (6.73), the following two controlled systems are equivalent:

$$
\dot{x}(t)=A(t) x(t)+B(t) u(t), \quad t \geq 0 .
$$

and

$$
\dot{z}(t)=\hat{A} z(t)+\hat{B}(t) u(t), \quad t \geq 0 .
$$

For each $t_{0} \geq 0, z_{0} \in \mathbb{R}^{n}$ and each control $u$, we denote by $z\left(\cdot ; t_{0}, z_{0}, u\right)$ the solution of (6.77) with the initial condition $z\left(t_{0}\right)=z_{0}$.

We claim that $[A(\cdot), B(\cdot)]$ is periodically stabilizable. By Theorem 1.2 and the equivalence of systems $(6.76)$ and (6.77), it suffices to show the null controllability of system (6.77) over $[0, T]$. For this purpose, we write $z_{k}\left(\cdot ; z_{k}, u\right)$ for the solution to the system:

$$
\dot{z}(t)=\hat{A} z(t)+b_{k} u(t), \quad t \in[k T / n,(k+1) T / n], \quad z(k T / n)=z_{k} .
$$

By Lemma 3.3.2 in [20] (see p. 91 in [20]), $\operatorname{Span}\left\{b_{k}, \hat{A} b_{k}, \ldots, \hat{A}^{n-1 b_{k}}\right\}$ is a controllable subspace of (6.78). In particular, for each $k \in\{0,1,2, \ldots, n-1\}$, there is a control $u_{k} \in L^{2}\left(\mathbb{R}^{+} ; \mathbb{R}^{1}\right)$ such that

$$
z_{k}\left((k+1) T / n ; b_{k}, u_{k}\right)=0 .
$$

From definitions of $z\left(\cdot ; t_{0}, z_{0}, u\right)$ and $z_{k}\left(\cdot ; z_{k}, u\right),(6.77),(6.78),(6.74),(6.75)$ and (6.79), it follows that

$$
\begin{aligned}
& z\left(T ; 0, e_{k+1}, \chi_{[k T / n,(k+1) T / n)} u_{k}\right) \\
= & \left.z\left(T ; k T / n, b_{k}, \chi_{[k T / n,(k+1) T / n)} u_{k}\right)\right) \\
= & \left.z\left(T ;(k+1) T / n, 0, \chi_{[k T / n,(k+1) T / n)} u_{k}\right)\right)=0,
\end{aligned}
$$

for all $k \in\{0,1,2, \ldots, n-1\}$. This leads to the null controllability of system (6.77) and completes the proof.

Remark 6.22. In the Proof of Theorem 6.20, we provide a way to construct a $B(\cdot) \in \mathcal{C B}_{A(\cdot)}$, with $m=1$, through utilizing the Lyapunov transform. 


\section{REFERENCES}

[1] H. Abou-Kandil, G. Freiling, V. Ionescu and G. Jank, Matrix Riccati equations: In control and systems theory. Systems \&f Control: Foundations \& Applications. Birkhäuser Verlag, Basel (2003).

[2] V.I. Arnol'd, Geometrical methods in the theory of ordinary differential equations. Translated from the Russian by Joseph Szücs. 2nd edition, vol. 250, Grundlehren der Mathematischen Wissenschaften [Fundamental Principles of Mathematical Sciences]. Springer-Verlag, New York (1988).

[3] V.I. Arnol'd, Ordinary Differential Equations. Springer-Verlag, Berlin, Heidelberg, New York, London, Paris, Tokyo, Hong Kong, Barcelona, Budapest (2003).

[4] V. Barbu and G. Wang, Feedback stabilization of periodic solutions to nonlinear parabolic-like evolution systems. Indiana Univ. Math. J. 54 (2005) 1521-1546.

[5] R. Brockett, A stabilization problem, Open problems in mathematical systems and control theory. Commun. Control Engrg. Ser. Springer, London (1999) 75-78.

[6] P. Brunovský, Controllability and linear closed-loop controls in linear periodic systems. J. Differ. Equs. 6 (1969) $296-313$.

[7] J.M. Coron, Control and nonlinearity, Mathematical Surveys and Monographs, vol. 136 of Amer. Math. Soc. Providence, RI (2007)

[8] G. Floquet, Sur les équations différentielles linéaires à coefficients périodiques (in French). Ann. Sci. École Norm. Sup. 12 (1883) $47-88$.

[9] D. Henry, Geometric theory of semilinear parabolic equations, vol. 840 of Lect. Notes Math. Springer-Verlag, Berlin, New York (1981).

[10] M.W. Hirsch and S. Smale, Differential equations, dynamical systems, and linear algebra, vol. 60 of Pure and Applied Mathematics. Academic Press [A subsidiary of Harcourt Brace Jovanovich, Publishers], New York, London (1974).

[11] M. Ikeda, H. Maeda and S. Kodama, Stabilization of linear systems. SIAM J. Control 10 (1972) 716-729.

[12] M. Ikeda, H. Maeda and S. Kodama, Estimation and feedback in linear time-varying systems: a deterministic theory. SIAM J. Control 13 (1975) 304-326.

[13] H. Kano and T. Nishimura, Periodic solution of matrix Riccati equations with detectability and stabilizability. Internat. J. Control 29 (1979) 471-487.

[14] H. Kano and T. Nishimura, Controllability, stabilizability, and matrix Riccati equations for periodic systems. IEEE Trans. Automat. Control 30 (1985) 1129-1131.

[15] G.A. Leonov, The Brockett stabilization problem (in Russian). Avtomat. i Telemekh (2001) 190-193; translation in Autom. Remote Control 62 (2001) 847-849.

[16] X. Li, J. Yong and Y. Zhou, Control Theory (in Chinese). Higher Education Press of P.R. China, Beijing (2009).

[17] A.M. Lyapunov, The general problem of the stability of motion, Translated from Edouard Davaux's French translation (1907) of the 1892 Russian original and edited by A.T. Fuller. With an introduction and preface by Fuller, a biography of Lyapunov by V.I. Smirnov, and a bibliography of Lyapunov's works compiled by J.F. Barrett. Lyapunov centenary issue. Reprint of Internat. J. Control 55 (1992) 521-790.

[18] I.G. Malkin, The stability theory of motion (in Russian). Nauk Press, Moscow (1966).

[19] R. Penrose, A Generalized inverse for matrices. Proc. Cambridge Philos. Soc. 51 (1955) 406-413.

[20] E.D. Sontag, Mathematical control theory: Deterministic finite-dimensional systems, 2nd edition, vol. 6 of Texts in Applied Mathematics. Springer-Verlag, New York (1998).

[21] W.H. Steeb and Y. Hardy, Matrix calculus and Kronecker product, A practical approach to linear and multilinear algebra, Second edition. World Scientific Publishing Co. Pte. Ltd., Hackensack (2011).

[22] W. Walter, Ordinary differential equations, Translated from the sixth German (1996) edition by Russell Thompson, vol. 182 of Graduate Texts in Mathematics Readings in Mathematics. Springer-Verlag, New York (1998).

[23] K. Yosida, Functional analysis, 6th edition, vol. 123 of Grundlehren der Mathematischen Wissenschaften [Fundamental Principles of Mathematical Sciences]. Springer-Verlag, Berlin, New York (1980). 